UNITED STATES DEPARTMENT OF THE INTERIOR

GEOLOGICAL SURVEY
HED. COLILEG

$$
\text { A16 } 31979
$$

WI. MNES R GELOOS?

\title{
ANALYSES OF ROCK AND STREAM-SEDIMENT SAMPLES FROM THE SUMDUM C-4 QUADRANGLE, ALASKA
}

By

\begin{abstract}
Allen L. Clark, David A. Brew, Donald A. Grybeck, and Raymond Wehr
\end{abstract}

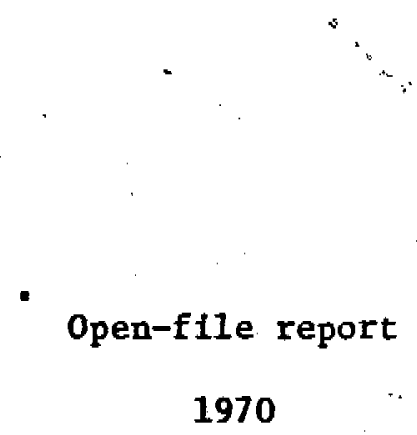

This report is preliminary and has not been edited or reviewed for conformity with Geological Survey standards 


\title{
ANALYSES OF ROCK AND STREAM-SEDIMENT SAMPLES FROM THE \\ SUMDUM C-4 QUADRANGLE, ALASKA \\ By
}

\author{
Allen L. Clark, David. A. Brew, Donald A. Grybeck, . \\ and Raymond Wehr
}

\section{INTRODUCTION}

Analytical data for 31 rock and 33 stream-sediment samples from the Sumdum $\mathrm{C}-4,1: 63,360$ scale quadrangle are presented in this report, together with a statistical treatment of the data. The samples were collected in 1969 as part of the Heavy Metals Program of the U.S. Geological Survey.

The most comprehensive discussion of the geology of the study area Is a report by A. F. Buddington and Theodore Chapin (1929). Known metalliferous lodes of the area are described and additional references to specific areas are given by Berg and Cobb (1967). Additional data is given in reports by Herbert and Race (1964) and Alaska Department of Mines (1950). Supplemental publications are being prepared on the general geology and mineral occurrences of the study area.

\section{Procedures and treatment of data}

Standard procedures were followed in the collection and preparation of samples.

Rock samples are primarily grab samples from mineral occurrences and outcrops. They were chosen for analysis to provide data on background, because they were in the area of mineral occurrences or streamsediment anomalies, because they were strongly iron stained, or contained visible sulfides.

Stream-sediment samples were generally collected from the active stream channel; where this was not possible, samples were collected from bank or terrace deposits adjacent to the channel.

Rock samples were crushed and pulverized and the minus 80 mesh fraction analyzed. Stream-sediment samples were dried, sieved, and the minus 80 mesh fraction analyzed. The minus 80 mesh fractions of the 
samples were analyzed for 30 elements by the six-step semiquantitative spectrographic method and for gold by the atomic absorption method. $1 /$

The spectrographic analyses were reported in percentage (pct) or parts per million (ppm) to the nearest number in the series $1.0,0.7$, $0.5,0.3,0.2,0.15,0.1$, etc. The precision of a reported value is approximately plus 100 percent or minus 50 percent. Analyses for gold by the atomic absorption method are accurate to \pm 100 percent. Minimum limits of determination for each element are given on page 4. Semiquantitative spectrographic analyses were done by $K$. J. Curry and atomic absorption analyses were done by R. L. Miller, R. B. Tripp, H. D. King, and A. L. Meier.

Locations of the rock and strean-sediment samples are shown on Plate 1. Rock sample descriptions are given in table 1 and rock sample analyses are tabulated in table 2 and stream-sediment analyses are tabulated in table 3 .

The results of the analyses of the rock and stream-sediment analyses have been processed by means of a computer program known as GEOSUM and are presented in tables 2 and 3 . The GEOSUM program is designed primarily for summarizing and tabulating geochemical data--especially data from semiquantitative spectrographic analyses (commonly referred to as six-step spectrographic analyses) by the laboratories of the U.S. Geological Survey.

The program output consists of: (a) a tabulation of the data, (b) histograms and cumulative frequency distributions for all elemerts except tungsten, and (c) a statistical summary which tncludes geometric means and geonetric deviations.

I/Analyses for 29 elements by semiquantitative analyses and for gold by atomic absorption are given in the tables. Semiquantitative analyses for gold are omitted. 
Table 1.--Description of rock, veln, and altered zone samples from the Sundum $\mathrm{C}-4$ quadrangle. (A11 samples are of representative materlal.) Sample localities are shown by sample number plotted on the accompanying map, Plate 1 .

Saniple No.

Lab. No.

Sample Description

1

2

2

2

2

2

2

2

2

2

3

4

5

6

7

7

8

9

10

10

11

12

13

14.

14

15

16

16

17

17

18

$A K D-892$
-894
-895
-896
-897
-898
-899
-900
-901
-902
-891
-890
-908
-893
-903
-904
-906
-907
-909
-910
-912
-913
-914
-870
-871
-883
-872
-873
-874
-875
-876

Biotite feldspar quartz

Sheared granodiorite and quartz fragments

Sheared granodiorite and quartz fragments

Quartz vein with minor pyrite

Quartz vein with minor pyrite

Euhedrral quartz crystals

Sheared and bleached granodiorite

Pyrite quartz vein

Pyrite quartz vein

Pyrite quartz vein

Biotite feldspar quartz gneiss

Biotite hornblende granodiorite

Biotite hornblende granodiorite

Biotite feldspar quartz.gneiss

Massive fibrous serpentine

Serpentinized dunite

Serpentinized dunite

Foliated granodiorite

Quartz vein

Quartz vein

Biotite hornblende granodiortte

Biotite hornblende granodiorite

Biotite quartzite

Biotite hornblende schist

Quartz vein

Pyritized quartz vein

Biotite hornblende schist

Iron-stained aplite dike

Biotite hornblende gneiss

Iron-stained biotite hornblende gneiss

Iron-stained biotite hornblende gneiss 


\section{Explanation of Tables..2 and 3}

Analytical results from rock and stream-sediment samples are given In Tables 2 and 3 as analytical values such as 7.0000 ppm, 10.0000 percent, etc., or as qualified values expressed as a letter. These letter codes are $\mathrm{N}=$ not detected, $L=$ less than specified limit of detection, $\mathbf{G}=$ greater than value shown, $\dot{B}=$ no data, $\mathrm{H}=$ interference. The term $T=$ trace, but does not occur in these data. Note that the right-most zero digits for each analytical value may or may not be significant. The specifled limits of detection are as follows:

\section{Specified limits of detection}

$\begin{array}{llllll}\text { FE PCT } & \text { MG PCT } & \text { CA PCT } & \text { TI PCT } & \text { MN PPM } & \text { AG PPM }\end{array}$

$\begin{array}{lccccc}0.05000 & 0.02000 & 0.05000 & 0.00200 & 20.00000 & 0.10000 \\ \text { AS PPM } & \text { AU PPM } & \text { B PPM } & \text { BA PPM } & \text { BE PPM } & \text { BI PPM } \\ 0.20000 & 0.02000 & 10.00000 & 20.00000 & 1.00000 & 10.00000 \\ \text { Co PPM } & \text { CR PPM } & \text { CU PPM } & \text { LA PPM } & \text { MO PPM } & \text { NB PPM } \\ 5.00000 & 5.00000 & 2.00000 & 20.00000 & 2.00000 & 10.00000 \\ \text { NI PPM } & \text { PB PPM } & \text { SB PPM } & \text { SC PPM } & \text { SN PPM } & \text { SR PPM } \\ 2.00000 & 10.00000 & 0.50000 & 5.00000 & 10.00000 & 50.00000 \\ \text { V PPM } & \text { W PPM } & \text { Y PPM } & \text { ZN PPM } & \text { ZR PPM } & \\ 5.00000 & 50.00000 & 5.00000 & 25.00000 & 10.00000 & \end{array}$

Semiquantitative spectrographic analyses by the U.S. Geological Survey are reported as geometric midpoints $(1.0,0.7,0.5,0.3,0.2$, $0.15,0.1$, etc.) of geometric backets having the boundaries $1.2,0.83$, $0.56,0.38,0.26,0.18,0.12,0.083$, etc. The frequency distributions and histograms are on logarithmic scales and are computed using these brackets as class intervals, for example:

\begin{tabular}{rcr} 
Reported value (ppm) & \multicolumn{2}{c}{ Limits } \\
1.0 & .83 & 1.2 \\
1.5 & 1.2 & 1.8 \\
2.0 & 1.8 & 2.6 \\
3.0 & 2.6 & 3.8 \\
5.0 & 3.8 & 5.6 \\
7.0 & 5.6 & 8.3 \\
100 & 8.3 & 12.0
\end{tabular}


On the histograms decimal numbers are shown as powers of 10 , for example:

$7.0 \mathrm{E}-01$ means $7.0 \times 10^{-1}$ or 0.7
$7.0 \mathrm{E} 00$ means $7.0 \times 10^{0}$ or 7.0
$7.0 \mathrm{E} 01$ means $7.0 \times 10^{1}$ or 70.0
$7.0 \mathrm{E} 02$ means $7.0 \times 10^{2}$ or 700.0
$7.0 \mathrm{E} 03$ means $7.0 \times 10^{3}$ or $7,000.0$

The histograms are constructed of X's, each of which represents 1 percent of the total number (309) of samples.

The histograms and the statistics given below them are derived only from data values within the ranges of analytical determination. ("analytical values"). The histograms are, therefore, incomplete, and the statistics are biased if data values qualified with $\mathrm{N}, \mathrm{L}, \mathrm{C}, \mathrm{T}$, or H codes are present. (See the histogram and statistics below it for $t i n$, which are calculated from only one sample.) Statistical estimates that are unbiased in this regard are given at the end of Table 1 . The geometric means is the antilogarithm of the arithmetic mean of the logs of the analyses and an estimate of "central tendency," or of a characteristic value $;$, of a frequency distribution that is approximately symmetrical on a $\log$ scale, and is therefore useful for characterizing many geochemical distributions. The geometric mean is not an estimate of geochemical abundance. The geometric deviation is the antilogarithm of the standard deviation of the logs of the analyses. See USGS Professional Paper 574-B for further discussion and USGS Bulletin 1147E, p. 20-23, for further discussion and explanation of geometric deviation.

In the computations performed to produce tle statistical sumary at the end of Tables 2 and 3 . all elements are ignored where one or more of the unqualified data values is less than the analytical limit of detection. specified on input or where any data values are qualified with the $G$ (greater than) code. Data values qualified with $B$ or $H$ are not used in the computations. Where none of the data values for an element are qualified the mean and deviation should be the same as those given in the preceding section. Where data are qualified with the codes $N, L$, or $T$, the estimates of geometric mean and deviation are based on a method by A. J. Cohen for treating censored distributions. The application of this method of geochemical problems is described in USGS professional Paper 574-B. The estimates are unbiased in a strict sense only where the data are derived from a lognormal parent population, but experiments have shown that large departures from this requirement may not greatly invalidate the results. Acceptance and use of the estimates, however, is the responsibility of the individual. 


\section{Selected References}

Aläska Dept. Mines, 1950, Rept. Comm1ssioner of Mines, bienniun Dec. 31, $1950,57 \mathrm{p}$.

Berg, H. C., and Cobb, E. H., 1967, Metalliferous lode deposits of Alaska: U.S. Geol. Survey Bu11. 1246, p. 164-184.

Buddington, A. F., and Chapin, Theodore, 1929, Geology and mineral deposits of southeastern Alaska: U.S. Geol. Survey Bull. 800, 398 p.

Herbert, C. F., and Race, W. H., 1964, Geochemical investigations of selected areas in southeastern Alaska, 1964: Alaska Div. Mines and Minerals Geochem. Rept. 1, 27 p.

Miesch, A. T., 1963, Distribution of elements in Colorado Plateau uranium deposits-A preliminary report: U.S. Geol. Survey Bull. 1147-E, $57 \mathrm{p}$ -

1967, Methods of computation for estimating geochemical abundance: U.S. Geo1. Survey Prof. Paper 574-B, 15 p. 
TABLE 2. SUMDUM C-4 ROCK SAMPLES

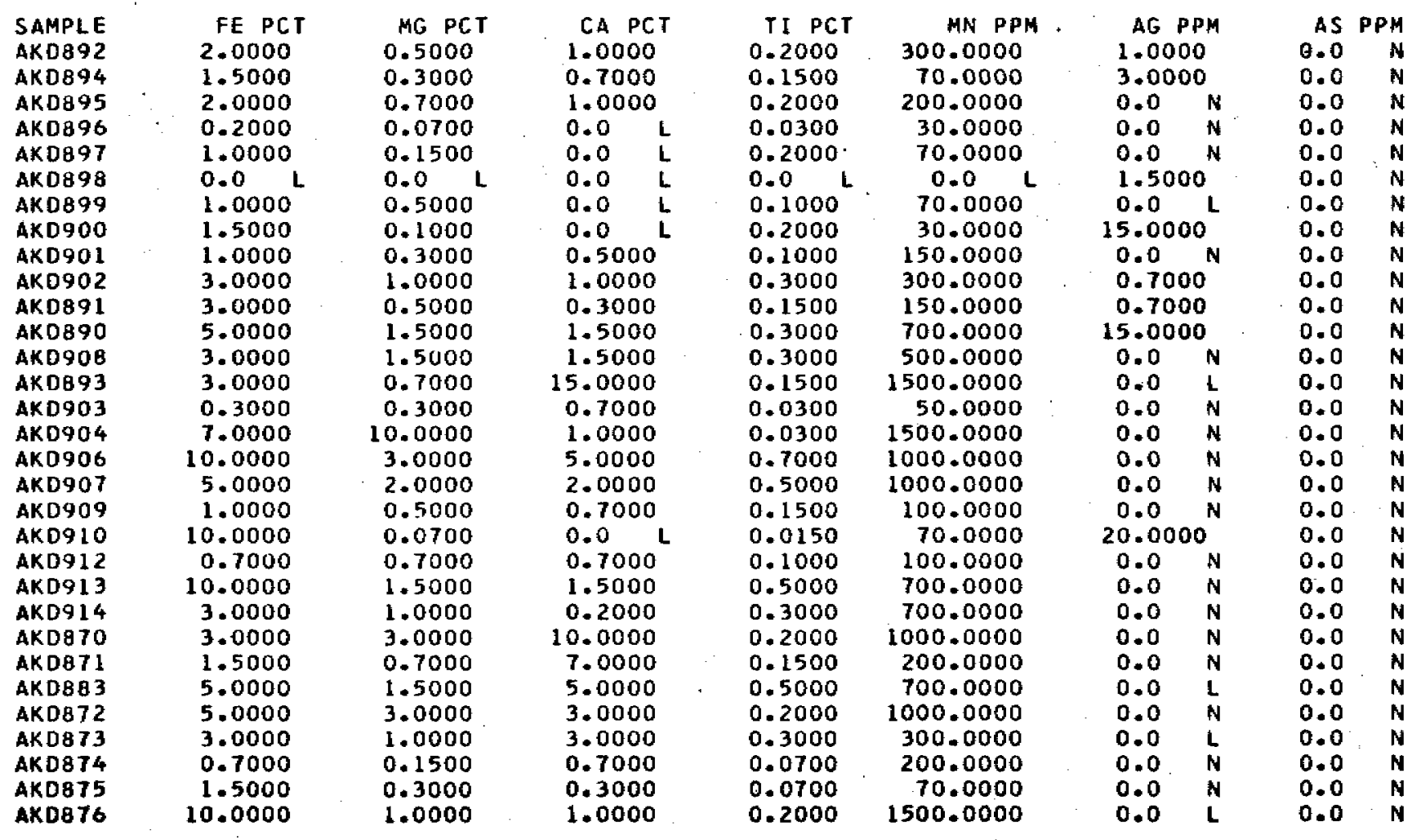

$\begin{array}{ll}\text { AU PP } \\ 0.0 & N \\ 0.0 & N \\ 0.0 & N \\ 0.0 & N \\ 0.0 & N \\ 0.0 & N \\ 0.0 & N \\ 0.0 & N \\ 0.0 & N \\ 0.0 & N \\ 0.0 & N \\ 0.0 & N \\ 0.0 & N \\ 0.0 & N \\ 0.0 & N \\ 0.0 & N \\ 0.0 & N \\ 0.0 & N \\ 0.0 & N \\ 0.0 & N \\ 0.0 & N \\ 0.0 & N \\ 0.0 & N \\ 0.0 & N \\ 0.0 & N \\ 0.0 & N \\ 0.0 & N \\ 0.0 & N \\ 0.0 & N \\ 0.0 & N \\ 0.0 & N\end{array}$

\begin{tabular}{|c|c|}
\hline 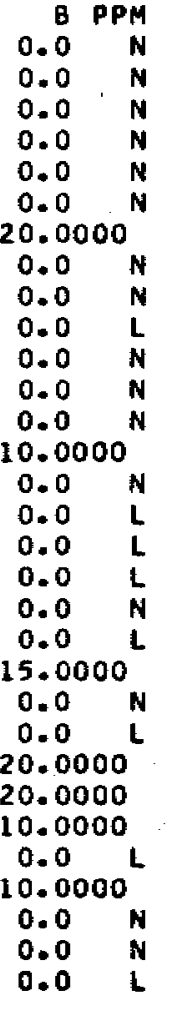 & $\begin{array}{c}\text { BA PPH } \\
1500.0000 \\
700.0000 \\
700.0000 \\
100.0000 \\
0.0 \\
0.0 \\
700.0000 \\
300.0000 \\
1500.0000 \\
1000.0000 \\
1500.0000 \\
1000.0000 \\
300.0000 \\
300.0000 \\
150.0000 \\
100.0000 \\
0.0 \\
700.0000 \\
1000.0000 \\
300.00000 \\
300.0000 \\
700.0000 \\
200.0000 \\
200.0000 \\
200.0000 \\
300.0000 \\
700.0000 \\
700.0000 \\
300.0000 \\
700.0000 \\
500.0000\end{array}$ \\
\hline
\end{tabular}


SUMDUM C-4 ROCK SAMPLES

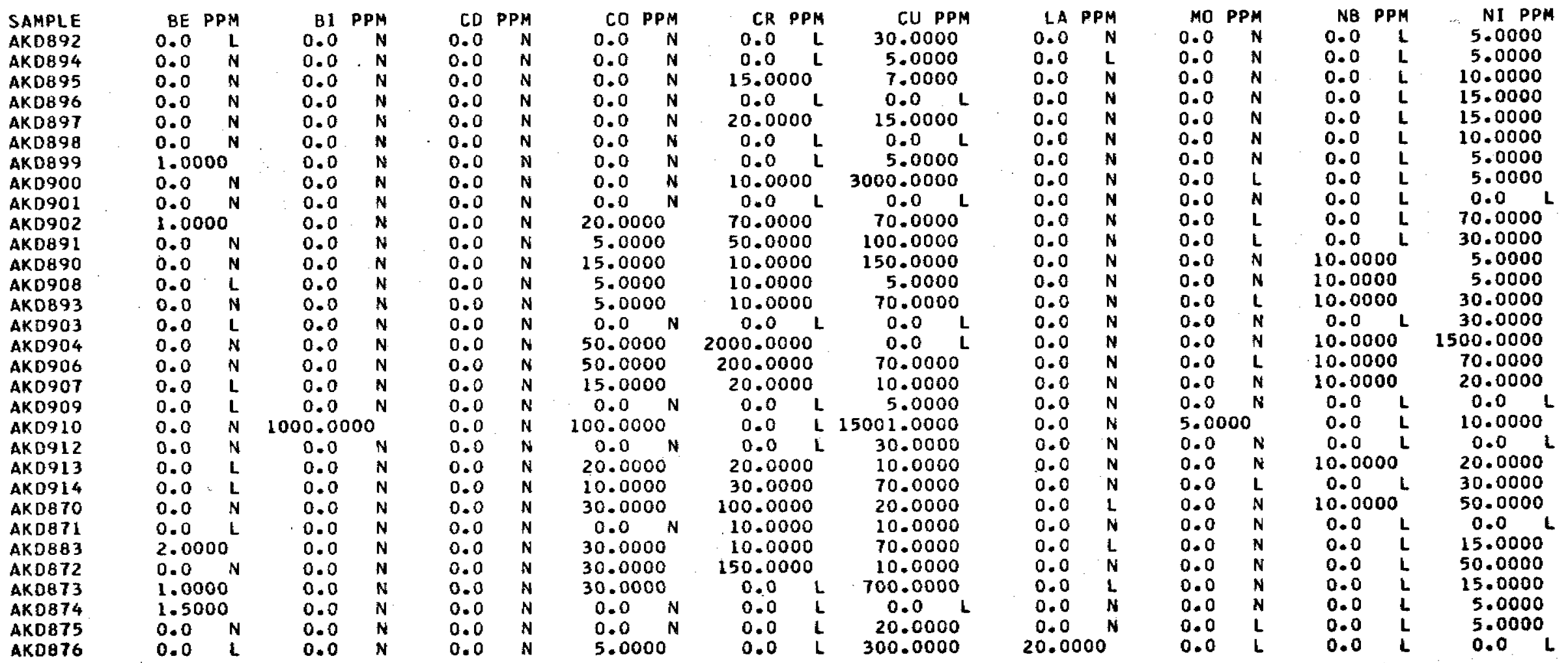


SUMDUM C -4 ROCK SAMPLES

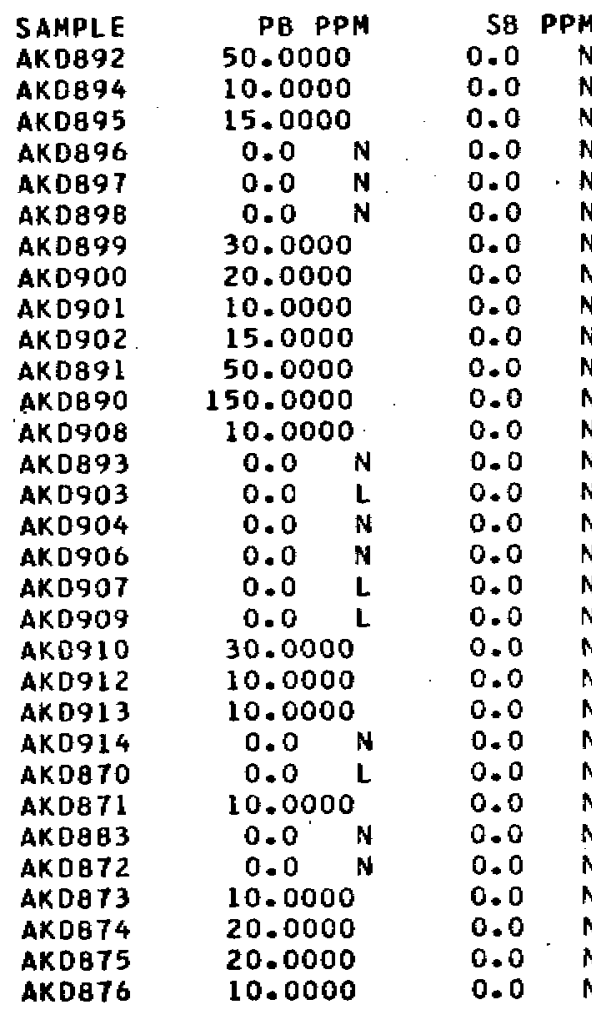

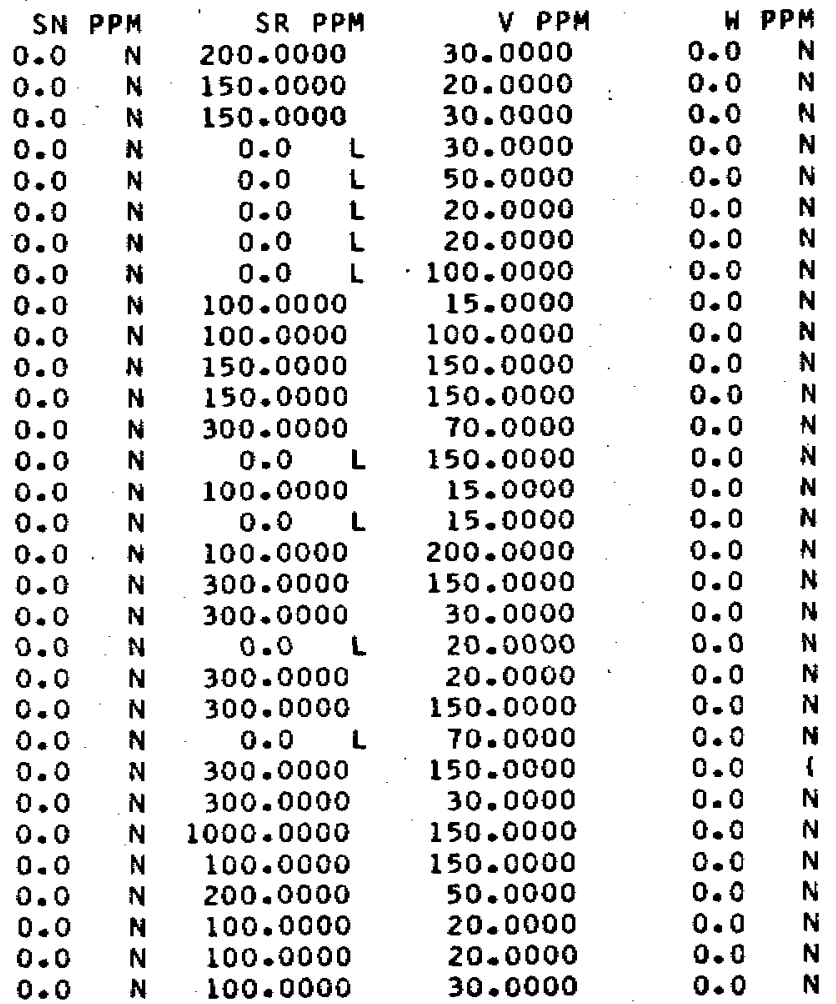

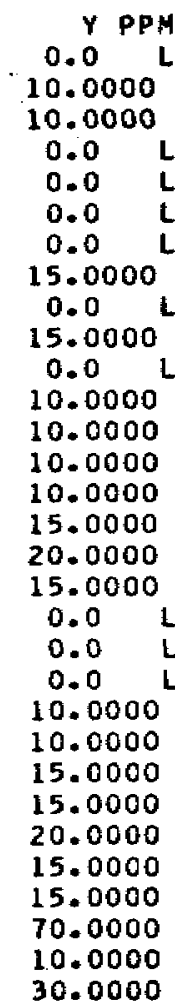

$\begin{array}{cc}2 N & P P M \\ 0.0 & L \\ 0.0 & N \\ 0.0 & N \\ 0.0 & N \\ 0.0 & N \\ 0.0 & N \\ 0.0 & N \\ 0.0 & N \\ 0.0 & N \\ 0.0 & L \\ 0.0 & L \\ 0.0 & L \\ 0.0 & N \\ 0.0 & N \\ 0.0 & N \\ 0.0 & N \\ 0.0 & N \\ 0.0 & L \\ 0.0 & N \\ 0.0 & N \\ 0.0 & N \\ 0.0 & L \\ 0.0 & N \\ 0.0 & N \\ 0.0 & N \\ 300.0000 \\ 200.0000 \\ 0.0 & N \\ 0.0 & N \\ 0.0 & N \\ 1500.0000 & \\ & \end{array}$

ZR PPM

70.0000

100.0000

150.0000

0.0

0.0

70.0000

50.0000

70.0000

70.0000

70.0000

70.0000

100.0000

70.0000

70.0000

50.0000

$0.0 \quad L$

70.0000

200.0000

70.0000

0.0

70.0000

30.0000

70.0000

70.0000

100.0000

70.0000

70.0000

70.0000

70.0000

70.0000

70.0000

200.0000 
SUMDUM C-4 ROCK SAMPLES

$\begin{array}{lc}\text { SAMPLE } & \text { AU PPM } \\ \text { AKOB92 } & 0.0200 \mathrm{~L} \\ \text { AKDQ94 } & 0.0200 \mathrm{~L} \\ \text { AKDB95 } & 0.0200 \mathrm{~L} \\ \text { AKD896 } & 0.0200 \mathrm{~L} \\ \text { AKDB97 } & 0.0200 \mathrm{~L} \\ \text { AKDB98 } & 0.0400 \\ \text { AKD899 } & 0.0400 \\ \text { AKD900 } & 0.0200 \mathrm{~L} \\ \text { AKD901 } & 0.0200 \mathrm{~L} \\ \text { AKD902 } & 0.0200 \mathrm{~L} \\ \text { AKD891 } & 0.0200 \mathrm{~L} \\ \text { AKDB90 } & 0.0200 \mathrm{~L} \\ \text { AKD90B } & 0.0200 \mathrm{~L} \\ \text { AKD893 } & 0.0200 \mathrm{~L} \\ \text { AKD903 } & 0.0200 \mathrm{~L} \\ \text { AKD904 } & 0.0200 \mathrm{~L} \\ \text { AKD906 } & 0.0200 \mathrm{~L} \\ \text { AKD907 } & 0.0200 \mathrm{~L} \\ \text { AKD909 } & 0.0200 \mathrm{~L} \\ \text { AKD910 } & 60.0000 \\ \text { AKD912 } & 0.0200 \mathrm{~L} \\ \text { AKD913 } & 0.0200 \mathrm{~L} \\ \text { AKD914 } & 0.0200 \mathrm{~L} \\ \text { AKDB70 } & 0.0200 \mathrm{~L} \\ \text { AKD871 } & 0.0200 \mathrm{~L} \\ \text { AKD883 } & 0.0200 \mathrm{~L} \\ \text { AKDB72 } & 0.0200 \mathrm{~L} \\ \text { AKD873 } & 0.0200 \mathrm{~L} \\ \text { AKD874 } & 0.0200 \mathrm{~L} \\ \text { AKD875 } & 0.0200 \mathrm{~L} \\ \text { AKD876 } & 0.0200 \mathrm{~L} \\ & \end{array}$


FREQUENCY TABLE FOR COLUMN

$$
1 \text { (FE PCT) }
$$

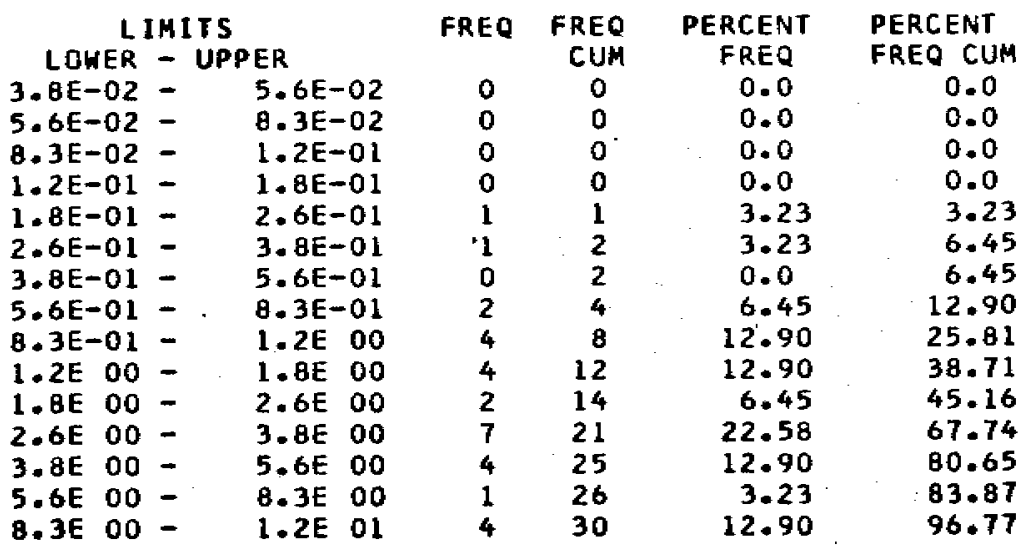

HISTOGRAM FOR CDLUMN

1 FE PCT ।

2. OE-OL $\times \times X$

3. OE-01 $\times \times \times$

5. OE-OL

7. OE- $01 \times \times \times \times \times \times$

1. OE OO $x \times x \times x \times x \times x \times x \times x$

1. 5E $00 \quad x \times x \times x \times x \times x \times x \times x$

2. OE $00 \times \times \times \times \times X$

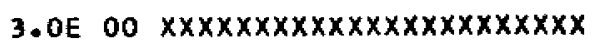

5. OE OO $\times x \times \times \times \times \times \times \times \times \times \times \times x$

7. OE $00 \mathrm{XXX}$

1. OE $01 \times \times \times \times \times \times \times \times \times \times \times \times \times$

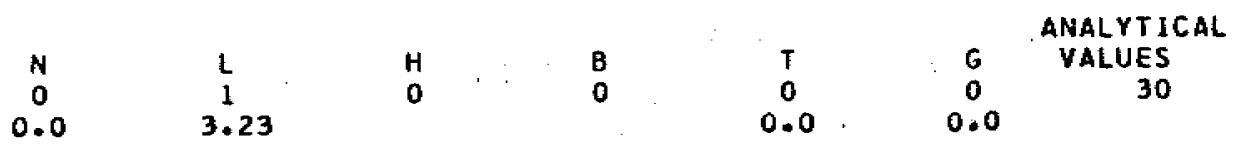

MAXIMUM $=1.00000 E 01$

MINIMUM $=2.00000 \mathrm{E}-01$

GEOMETRIC MEAN $=2.28307 E 00$

GEOMETRIC DEVIATION $=2.73103 E 00$ 
FREQUENCY TABLE FOR COLUMN

2 ( MG PCT)

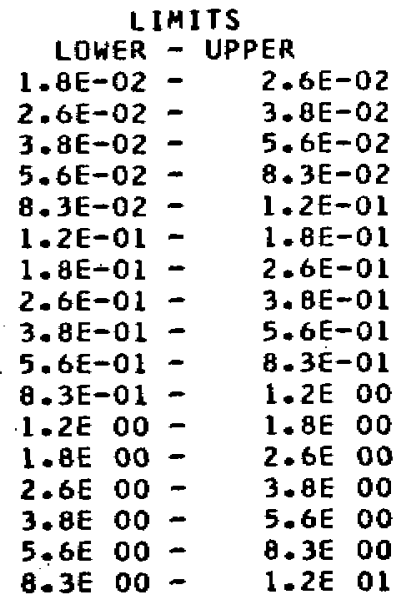

$\begin{array}{cr}\text { FREQ } & \text { FRE } \\ & \text { CU } \\ 0 & 0 \\ 0 & 0 \\ 0 & 0 \\ 2 & 2 \\ 1 & 3 \\ 2 & 5 \\ 0 & 5 \\ 4 & 9 \\ 4 & 13 \\ 4 & 17 \\ 4 & 21 \\ 4 & 25 \\ 1 & 26 \\ 3 & 29 \\ 0 & 29 \\ 0 & 29 \\ 1 & 30\end{array}$

PERCENT

PERCENT

CUN
0
0
0
2
3
5
5
9
13
17
21
25
26
29
29
29
30

FREQ FREQ CUM

$0.0 \quad 0.0$

$0.0 \quad 0.0$

$\begin{array}{ll}6.45 & 6.45 \\ 3.23 & 9.68\end{array}$

$6.45 \quad 16.13$

$0.0 \quad 16.13$

$12.90 \quad 29.03$

12.90

$12.90 \quad 54.84$

$12.90 \quad 67.74$

$12.90 \quad 80.65$

$3.23 \quad 83.87$

$9.68 \quad 93.55$

$0.0 \quad 93.55$

$0.0 \quad 93.55$

$3.23 \quad 96.77$

HISTOGRAM FOR COLUMN

2 I MG PCT J

7. OE-02 $\times \times \times \times \times \times$

1. $0 E-01 \times \times X$

1. 5E-01 $\times \times \times \times \times X$

2. $0 E-01$

3. OE-01 $\times \times \times \times \times \times \times \times \times \times \times \times x$

5. $0 E-01 \times \times \times \times \times \times \times \times \times \times \times \times x$

7.0E-01 $\times \times \times \times \times \times \times \times \times \times \times \times \times x$

1. OE $00 \quad x \times x \times x \times x \times x \times x \times x$

1.5E $00 \quad \times \times \times \times \times \times \times \times \times \times \times \times x$

2. OE $00 \times \times x$

3.0E $00 \quad \times \times \times \times \times \times \times \times \times x$

5.0E 00

7. OE OO

1.0E O1 $\times \times x$

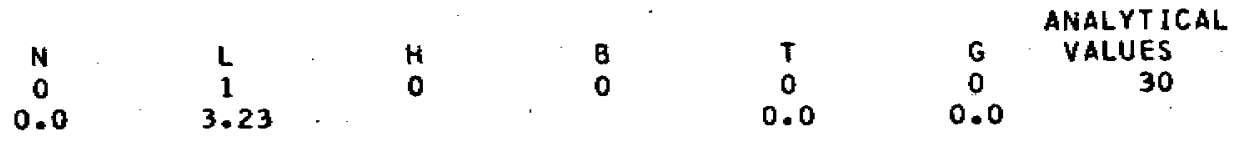

MAXIMUM $=1.00000 E 01$

MINIMUM $=7.00000 E-02$ 
GEOMETRIC MEAN $=6.58866 E-01$

\section{GEOMETRIC DEVIATION $=3.22257 E 00$}


GEOMETRIC MEAN $=6.58866 E-01$

GEOMETRIC DEVIATION $=3.22257 E$ 00 
FREQUENCY TABLE FOR COLUMN 31 CA PCT,

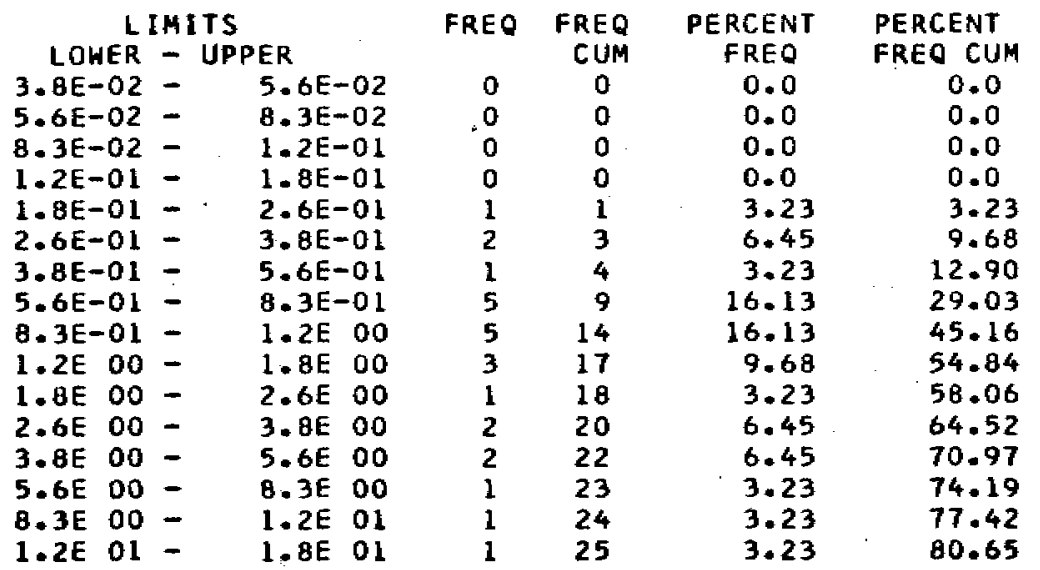

HISTOGRAM FOR COLUMN 3 ( CA PCT ,

2. OE-01 $\times \times X$

3. $0 E-01 \times \times \times \times \times \times$

5. $0 E-01 \times X X$

7.0E-01 $\times \times \times \times \times \times \times \times \times \times \times \times \times \times \times \times$

1. $0 E$ 00 $\times \times \times \times \times \times \times \times \times \times \times \times \times \times \times \times$

1.5E $00 \quad \times \times \times \times \times \times \times \times \times \times$

2. OE $00 \times x \times$

3. OE $00 \times \times \times \times \times x \times$

5.0E $00 \quad X \times \times \times \times X$

7. OE OO $\times \times \times$

1. OE $01 \times \times X$

1. $5 E 01 \times \times x$

$\begin{array}{ccccccc}N & \text { L } & H & \text { B } & T & & \text { G ANALYTICAL } \\ 0 & 6 & 0 & 0 & 0 & 0 & 25 \\ 0.0 & 19.35 & & & 0.0 & 0.0 & \end{array}$

MAXIMUM $=1.50000 E 01$

MINIMUM $=2.00000 E-01$

GEOMETRIC MEAN $=1.36551 E 00$

GEOMETRIC DEVIATION $=3.01155 E 00$ 
FREQUENCY TABLE FOR COLUMN

4 II PCT

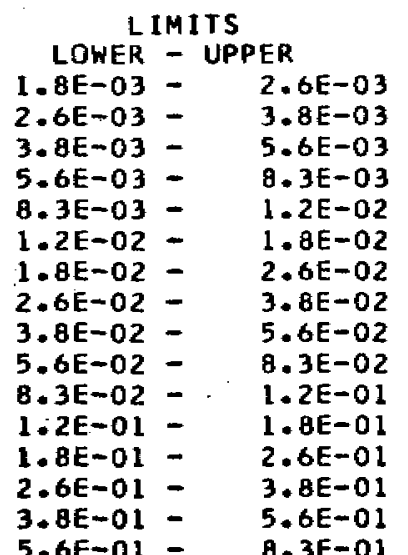

FREQ FREQ PERCENT

$\begin{array}{cccc}\text { FREQ } & \text { FREQ } & \text { PERCENT } & \text { PERCENT } \\ & \text { CUM } & \text { FREQ } & \text { FREQ CUM } \\ 0 & 0 & 0.0 & 0.0\end{array}$

.8E-03 - 2.6E-03

$2.6 \mathrm{E}-03-3.8 \mathrm{E}-03$

5.6E-03

1. $2 E-02$

$1.8 \mathrm{E}-02$

6E-02

$8 E-02$

. $3 E-02$

. $2 E-01$

BE- 01

5. $6 E-01$

0.0

0.0

0.0

0.0

3.23

0.0

9.68

0.0

6.45

9.68

16.13

22.58

16.13

9.68

0.0
0.0

0.0

0.0

0.0

3.23

3.23

12.90

12.90

19.35

29.03

45.16

67.74

83.87

93.55

5.6E-01 -

'

4 ITIPCT ।

HISTOGRAM FOR COLUMN

96.77

1. 5E-02 $\times \times x$

2. OE-02

3. $0 \mathrm{E}-0.2 \times \times \times \times \times \times \times \times \times x$

5. OE- 02

en

7. OE-02 $\times \times \times \times \times x$

1. OE-01 $\times \times \times \times \times \times \times \times \times \times$

1.5E-01 $\times \times \times \times \times \times \times \times \times \times \times \times \times \times \times \times$

2. $0 E-01 \quad x \times x \times x \times x \times x \times x \times x \times x \times x \times x \times x \times x$

3. OE-0.1 $\times \times \times \times \times \times \times \times \times \times \times \times \times \times \times \times$

5. OE-O1 $\times \times \times \times \times \times \times \times \times \times$

7. OE-01 XXX

$\begin{array}{ccccccc}N & \text { L } & H & & & & \text { ANALYTICAL } \\ 0 & 1 & 0 & T & G & \text { VALUES } \\ 0.0 & 3.23 & 0 & 0 & 0 & 0 & 30\end{array}$

MAXIMUM $=7.00000 E-01$

MINIMUM $=1.50000 E-02$

GEOMETRIC MEAN $=1.53851 \mathrm{E}-01$

GEOMETRIC DEVIATION $=2.50300 E 00$ 
FREQUENCY TABLE FOR COLUMN

$5 \mid$ MN PPM )

\begin{tabular}{|c|c|c|c|c|}
\hline & LER & IMI & & \\
\hline & 00 & - & $1.2 \mathrm{E}$ & 01 \\
\hline $1.2 \mathrm{E}$ & 01 & - & $1.8 E$ & 01 \\
\hline $1.8 \mathrm{E}$ & 01 & - & $2.6 E$ & 01 \\
\hline $6 E$ & 01 & - & $3.8 E$ & 01 \\
\hline $\mathrm{BE}$ & 01 & - & $5.6 \mathrm{E}$ & 01 \\
\hline $6 \mathrm{E}$ & 01 & - & $8.3 \mathrm{E}$ & 01 \\
\hline $3 E$ & 01 & - & $1.2 E$ & 02 \\
\hline & 02 & - & $1.8 E$ & 02 \\
\hline & 02 & - & $2.6 \mathrm{E}$ & 02 \\
\hline $6 E$ & 02 & - & $8 E$ & 02 \\
\hline $\mathrm{BE}$ & 02 & - & $6 \mathrm{E}$ & 02 \\
\hline & 02 & 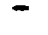 & 3E & 02 \\
\hline $2 E$ & $\begin{array}{l}02 \\
03\end{array}$ & $\overline{-}$ & $\begin{array}{l}1.2 \mathrm{E} \\
1.8 \mathrm{E}\end{array}$ & $\begin{array}{l}03 \\
03\end{array}$ \\
\hline
\end{tabular}

FREQ
0
0
0
0
2
1
5
2
2
3
3
1
4
4
3

$\begin{array}{ccc}\text { FREO } & \text { PERCENT } & \text { PERCENT } \\ \text { CUM } & \text { FREQ } & \text { FREQ CUM } \\ 0 & 0.0 & 0.0 \\ 0 & 0.0 & 0.0 \\ 0 & 0.0 & 0.0 \\ 2 & 6.45 & 6.45 \\ 3 & 3.23 & 9.68 \\ 8 & 16.13 & 25.81 \\ 10 & .6 .45 & 32.26 \\ 12 & 6.45 & 38.71 \\ 15 & 9.68 & 48.39 \\ 18 & 9.68 & 58.06 \\ 19 & 3.23 & 61.29 \\ 23 & 12.90 & 74.19 \\ 27 & 12.90 & 87.10 \\ 30 & 9.68 & 96.77\end{array}$

HISTOGRAM FOR COLUMN

51 MN PPH

3. OE $01 \times \times \times \times \times \times$

5. OE O1 XXX

7. OE Ol $\times x \times x \times x \times \times \times x \times \times \times \times \times x$

1.0E $02 \times \times \times \times \times \times$

1. $5 \mathrm{E} 02 \times \times \times \times \times \times$

2.0E $02 \times \times \times \times \times \times \times \times \times x$

3.0E $02 \quad x \times \times \times \times \times \times \times \times x$

5.OE O2 $\times X X$

T. OE $02 \quad \times \times \times \times \times \times \times \times \times \times \times \times \times x$

1. OE $03 \times \times \times \times \times \times \times \times \times \times \times \times \times \times$

1.5E $03 \times \times \times \times \times \times \times \times \times \times$

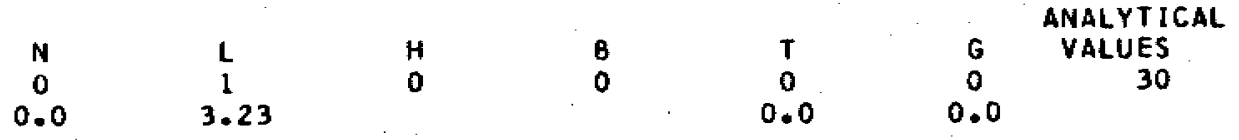

MAXIMUM $=1.50000 E 03$

MINIMUM $=3.00000 E$ OI

GEOMETRIC MEAN $=2.54543 E 02$

GEOMETRIC DEVIATION $=3.43519$ EO 
FREQUENCY TABLE FOR COLUMN

5 I MN PPM I

\begin{tabular}{|c|c|c|c|c|}
\hline & & $M I$ & & \\
\hline 0 & NER & & PER & \\
\hline 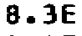 & 00 & - & $1.2 \mathrm{E}$ & 01 \\
\hline $2 \mathrm{E}$ & 01 & - & $1.8 \mathrm{E}$ & 01 \\
\hline & 01 & - & $6 \mathrm{E}$ & 01 \\
\hline & 01 & - & $B E$ & 01 \\
\hline $8 \mathrm{E}$ & 01 & - & $5.6 \mathrm{E}$ & 01 \\
\hline & ol & $E$ & $3 E$ & 01 \\
\hline & 01 & $\overline{-}$ & $2 \mathrm{E}$ & 02 \\
\hline & 02 & - & ge & 02 \\
\hline $10 t$ & 02 & - & $3.8 \mathrm{E}$ & 02 \\
\hline$B E$ & 02 & - & $5.6 \mathrm{E}$ & 02 \\
\hline & 02 & 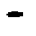 & $.3 E$ & 02 \\
\hline & $\begin{array}{l}02 \\
03\end{array}$ & - & $\begin{array}{l}2 \mathrm{E} \\
8 \mathrm{E}\end{array}$ & 03 \\
\hline & & & & \\
\hline
\end{tabular}

$\begin{array}{cc}\text { FREQ } & \text { FREQ } \\ & \text { CUA } \\ 0 & 0 \\ 0 & 0 \\ 0 & 0 \\ 2 & \\ 1 & \\ 5 & \\ 2 & 1 \\ 2 & 1 \\ 3 & 1 \\ 3 & 1 \\ 1 & 1 \\ 4 & 2 \\ 4 & 2 \\ 3 & 3\end{array}$

$\begin{array}{cc}\text { PERCENT } & \text { PERCENT } \\ \text { FREQ } & \text { FREQ CUM } \\ 0.0 & 0.0 \\ 0.0 & 0.0 \\ 0.0 & 0.0 \\ 6.45 & 6.45 \\ 3.23 & 9.68 \\ 16.13 & 25.81 \\ 6.45 & 32.26 \\ 6.45 & 38.71 \\ 9.68 & 48.39 \\ 9.68 & 58.06 \\ 3.23 & 61.29 \\ 12.90 & 74.19 \\ 12.90 & 87.10 \\ 9.68 & 96.77\end{array}$

HISTOGRAM FOR COLUMN

3. OE $01 \quad x \times \times \times \times x$

5. OE $01 \times X X$

7. OE O1 $\times \times \times \times \times \times \times \times \times \times \times \times \times \times \times x$

1. OE $02 \times \times \times \times \times X$

1.5E $02 \times \times \times \times \times x$

2.0E $02 \times \times \times \times \times \times \times \times \times x$

3. 0E $02 \quad x \times x \times \times \times \times \times \times x$

5. OE $02 \times x X$

7. OE $02 \times \times \times \times \times \times \times \times \times \times \times \times x$

1. OE $03 \times \times \times \times \times \times \times \times \times \times \times \times \times$

1.5E $03 \times \times \times \times \times \times \times \times \times x$

$\begin{array}{ccccccc}N & t & H & 0 & T & & \text { ANALYTICAL } \\ 0 & 1 & 0 & 0 & 0 & 0 & \text { VALUES } \\ 0.0 & 3.23 & & 0 & 0.0 & 0.0 & 30\end{array}$

MAXIMUM $=1.50000 E 03$

MINIMUM $=3.00000 \mathrm{E}$ OL

GEOMETRIC MEAN $=2.54543$ E 02

GEOMETRIC DEVIATIDN $=3.43519 E 00$ 
FREOUENCY TABLE FOR COLUMN

6 I AG PPM ।

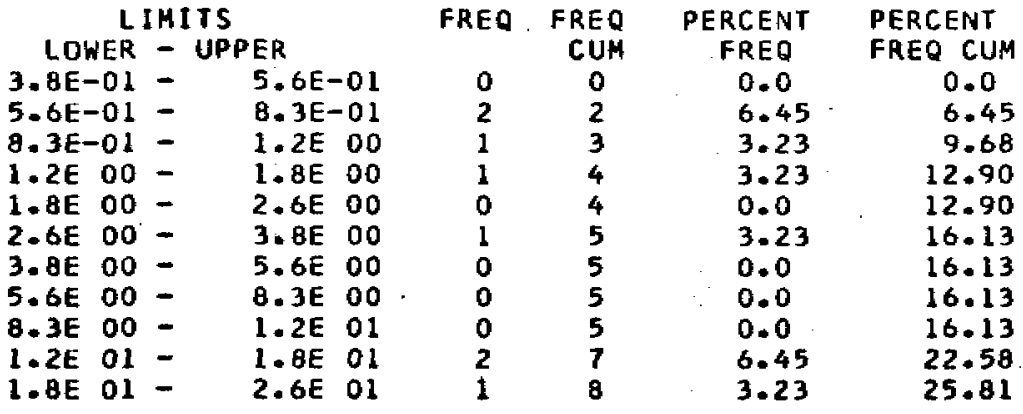

HISTOGRAM FOR COLUMN

6 I AG PPM I

7. OE-01 $\times \times \times \times \times \times$

1. 0 E $00 \times \times \times$

1. SE $00 \times \times \times$

2. OE 00

3. 0 E $00 \times x \times$

5.0E 00

7. OE 00

$1.0 E$ Ol

1.5E $01 \quad \times \times \times \times \times x$

2.0E $01 \times \times x$

\begin{tabular}{|c|c|c|c|c|c|c|}
\hline$N$ & $L$ & $H$ & $\theta$ & $T$ & $G$ & $\begin{array}{l}\text { ANALYT ICAL } \\
\text { VALUES }\end{array}$ \\
\hline $\begin{array}{c}18 \\
58.06\end{array}$ & $16^{5} 13$ & 0 & 0 & $\begin{array}{c}0 \\
0.0\end{array}$ & $\begin{array}{c}0 \\
0.0\end{array}$ & B \\
\hline
\end{tabular}

MAXIMUM $=2.00000 E 01$

MINIMUM $=7.00000 E-01$

GEOMETRIC MEAN $=3.15920 E 00$

GEOMETRIC DEVIATION $=4.25552 E 00$ 
FREOUENCY TABLE FDR COLUMN

6 I AG PPM |

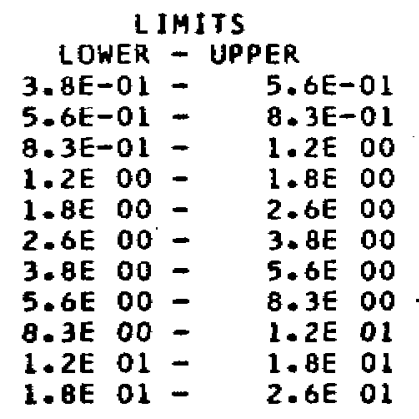

$\begin{array}{cc}\text { FREQ } & \text { FR } \\ & \text { C } \\ 2 & \\ 1 & \\ 1 & \\ 0 & \\ 1 & \\ 0 & \\ 0 & \\ 0 & \\ 2 & \\ 1 & \end{array}$

PERCENT FREQ FREO CUM $0.0 \quad 0.0$ 6.45 - 6.45 $3.23 \quad 9.68$ $3.23 \quad 12.90$ $0.0 \quad 12.90$ $3.23 \quad 16.13$ $0.0 \quad 16.13$ $0.0 \quad 16.13$ $0.0 \quad 16.13$ $0.0 \quad 16.13$ $6.45 \quad 22.58$

HISTOGRAM FOR COLUMN

6 A AG PPH I

\section{OE $-01 \times \times \times \times \times \times$}

$1.0 \mathrm{E} 00 \mathrm{XXX}$

1. $5 \mathrm{E} 00 \times \times \times$

2.0E 00

3. OE $00 \times \times \times$

5.OE 00

7. OE 00

1. OE 01

1. $5 \mathrm{E} 01 \times \times \times \times \times \times$

$2.0 E 01 \times \times X$

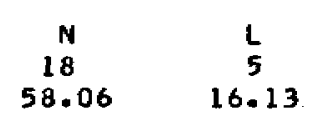

$H$
0

$B$
0

$T$
0
0.0

ANALYT ICAL

VALUES

MAXIMUM $=2.00000 E 01$

MINIMUM $=7.00000 E-01$

GEOMETRIC MEAN $=3.15920$ OO

GEOMETRIC DEVIATION $=4.25552 E 00$ 
fREQUENCY TABLE FOR COLUMN

71 AS PPM 1

\begin{tabular}{|c|c|c|c|c|c|}
\hline $\begin{array}{c}\text { LIMITS } \\
\text { LOWER - UPPER }\end{array}$ & FREQ & $\begin{array}{l}\text { FREQ } \\
\text { CUM }\end{array}$ & $\begin{array}{c}\text { PERCENT } \\
\text { FREQ }\end{array}$ & $\begin{array}{l}\text { PERCENT } \\
\text { FREO CUM }\end{array}$ & \\
\hline $\begin{array}{l}N \\
31 \\
* * * *\end{array}$ & $\begin{array}{l}H \\
0\end{array}$ & $\begin{array}{l}B \\
0\end{array}$ & $\begin{array}{c}T \\
0 \\
0.0\end{array}$ & $\begin{array}{c}G \\
0 \\
0.0\end{array}$ & $\begin{array}{c}\text { ANALYT ICAL } \\
\text { VALUES } \\
0\end{array}$ \\
\hline
\end{tabular}

MAXIMUM $=-9.99900 E 48$

I MIMIMUM $=9.99900 E 48$

GEDMETRIC MEAN $=9.99900$ E 48

GEOMETRIC DEVIATION $=9.99900 E 48$ 
FREQUENCY TABLE FOR COLUMN

$8 \mid$ AU PPH I

\begin{tabular}{|c|c|c|c|c|c|}
\hline $\begin{array}{c}\text { LIMITS } \\
\text { LOWER - UPPER }\end{array}$ & FREQ & $\begin{array}{l}\text { FREQ } \\
\text { CUH }\end{array}$ & $\begin{array}{l}\text { PERCENT } \\
\text { FREO }\end{array}$ & $\begin{array}{l}\text { PERCENT } \\
\text { FREQ CUM }\end{array}$ & \\
\hline $\begin{array}{c}N \\
31 \\
* * * *\end{array}$ & $\begin{array}{l}\mathrm{H} \\
0\end{array}$ & $\begin{array}{l}B \\
0\end{array}$ & $\begin{array}{c}T \\
0 \\
0.0\end{array}$ & $\begin{array}{c}6 \\
0 \\
0.0\end{array}$ & $\begin{array}{c}\text { ANALYT ICAL } \\
\text { VALUES } \\
0\end{array}$ \\
\hline
\end{tabular}

MAXIMUM $=-9.99900 E 48$

MINIMUM $=9.99900 E 48$

GEOMETRIC MEAN $=9.99900 E 40$

GEOMETRIC DEVIATION $=9.99900 E 48$ 


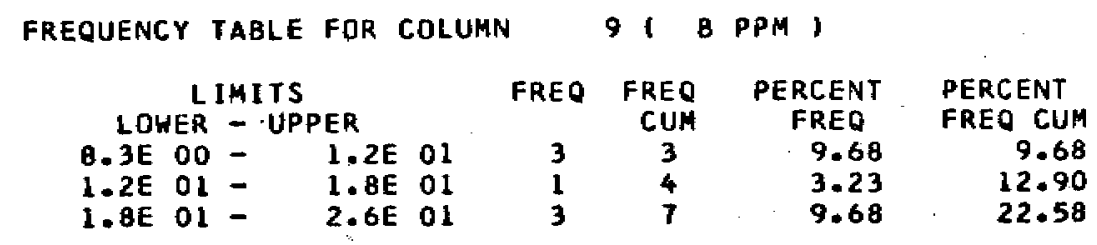

HISTOGRAM FOR COLUMN ' 91 B PPM ,

1.0E $01 \times \times \times \times \times \times \times \times \times \times$

1.5E O1 $\times \times x$

2.0E $01 \times \times \times \times \times \times \times \times \times \times$

\begin{tabular}{|c|c|c|c|c|c|c|}
\hline $\begin{array}{r}N \\
16\end{array}$ & $\begin{array}{l}\mathbf{L} \\
\mathbf{B}\end{array}$ & $\begin{array}{l}H \\
O\end{array}$ & $\begin{array}{l}B \\
0\end{array}$ & $\begin{array}{l}T \\
0\end{array}$ & $\begin{array}{l}\mathbf{G} \\
0\end{array}$ & $\begin{array}{c}\text { ANALYT ICAL } \\
\text { VALUES } \\
7\end{array}$ \\
\hline 51.61 & 25.81 & & & 0.0 & 0.0 & \\
\hline
\end{tabular}

MAXIMUM $=2.00000 E 01$

MINIMUM $=1.00000 E 01$

GEOMETRIC MEAN $=1.42615 \mathrm{E} 01$

GEOHETRIC DEVIATION $=1.42523 E 00$

$\tilde{a}$ 
FREQUENCY TABLE FOR COLUMN

10 ( BA PPM ।

\begin{tabular}{|c|c|c|c|c|c|c|c|c|}
\hline 3 & 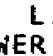 & MI & ER & & FREO & $\begin{array}{r}\text { FREQ } \\
\text { CUM }\end{array}$ & $\begin{array}{c}\text { PERCENT } \\
\text { FREQ }\end{array}$ & $\begin{array}{l}\text { PERCENT } \\
\text { FREO CUM }\end{array}$ \\
\hline BE & 01 & - & $2.6 \mathrm{E}$ & 01 & 0 & 0 & 0.0 & 0.0 \\
\hline $6 \mathrm{E}$ & 01 & - & $3.8 E$ & 01 & 0 & 0 & 0.0 & 0.0 \\
\hline$\bullet 05$ & 01 & - & $5.6 \mathrm{E}$ & 01 & 0 & 0 & 0.0 & 0.0 \\
\hline 6 & 01 & - & $B .3 E$ & 01 & 0 & 0 & 0.0 & 0.0 \\
\hline $3.3 E$ & 01 & - & $1.2 \mathrm{E}$ & 02 & 2 & 2 & 6.45 & 6.45 \\
\hline $1.2 \mathrm{E}$ & 02 & - & $1.8 \mathrm{E}$ & 02 & 1 & 3 & 3.23 & 9.68 \\
\hline $1.8 \mathrm{E}$ & 02 & - & $2.6 \mathrm{E}$ & 02 & 3 & 6 & 9.68 & 19.35 \\
\hline $2.6 \mathrm{E}$ & 02 & - & $3.8 \mathrm{E}$ & 02 & 7 & 13 & 22.58 & 94 \\
\hline $\mathbf{F}$ & 02 & - & $5.6 \mathrm{E}$ & 02 & 1 & 14 & 3.23 & 16 \\
\hline 6 & 02 & - & $8.3 E$ & 02 & 8. & 22 & 25.81 & \\
\hline $3 F$ & 02 & - & $1.2 \mathrm{E}$ & 03 & 3 & 25 & 9.68 & 80.65 \\
\hline & 03 & $=$ & $1.8 \mathrm{E}$ & 03 & 3 & 28 & 9.68 & 90.32 \\
\hline
\end{tabular}

HISTOGRAM FOR COLUHN 10 ( BA PPM I

1.0E $02 \times \times \times \times \times \times$

1.5E $02 \times \times \times$

2. OE $02 \times \times \times \times \times \times \times \times \times x$

3.0E $02 \quad x \times x \times x \times x \times x \times x \times x \times x \times x x \times x x \times x$

5. OE $02 \times \times X$

7.0E $02 \quad x \times x \times \times \times \times \times \times \times \times \times \times \times \times \times \times \times \times \times \times \times \times \times \times x$

1.0E $03 \times \times \times \times \times \times \times \times \times \times x$

1.5E $03 \times \times \times \times \times \times \times \times \times \times$

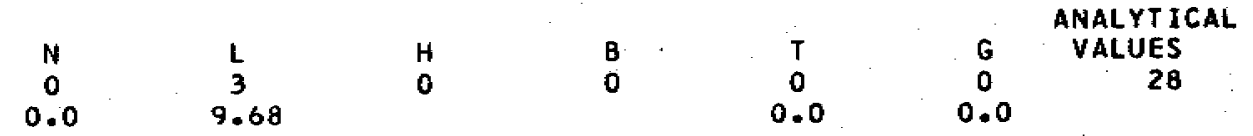

MAXIMUM $=1.50000 E 03$

MINIMUM $=1.00000 E 02$

GEOMETRIC MEAN $=4.54348 E 02$

GEOMETRIC DEVIATION $=2.19512 E 00$ 
FREQUENCY TABLE FOR COLUMN 11 ( BE PPM ।

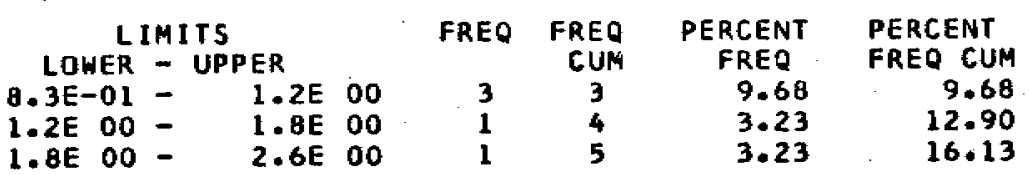

HISTOGRAM FOR COLUMN 11 ( BE PPM )

1. OE $00 \times \times \times \times \times \times \times \times \times \times$

1.5E $00 \times \times X$

$2.0 E 00 \times x \times$

\begin{tabular}{ccccccc}
$N$ & & & & & \multicolumn{2}{c}{ ANALYTICAL } \\
17 & $\mathbf{N}$ & $\mathrm{H}$ & $\mathrm{B}$ & $\mathrm{T}$ & $\mathrm{G}$ & VALUES \\
54.84 & 29.03 & 0 & 0 & 0 & 0 & 5
\end{tabular}

MAXIMUM $=2.00000 E 00$

MINIMUM $=1.00000 E 00$

GEOMETRIC MEAN $=1.24573$ E 00

GEOHETRIC DEVIATION $=1.37382 E 00$ 
FREQUENCY TABLE FOR COLUMN

12 (BI PPM )

\begin{tabular}{|c|c|c|c|c|}
\hline & & MI & & \\
\hline & NER & & PER & \\
\hline$B .3 E$ & 00 & & $1.2 \mathrm{E}$ & 01 \\
\hline $2 \mathrm{E}$ & 01 & 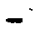 & $1.8 \mathrm{E}$ & 0 \\
\hline $8 \mathrm{E}$ & 01 & - & $2 \cdot 6 E$ & 0 \\
\hline OE & 01 & - & $8 E$ & 01 \\
\hline & 01 & - & GE & 0 \\
\hline & 01 & - & $8.3 E$ & 0 \\
\hline$E$ & 01 & - & $1.2 \mathrm{E}$ & 0 \\
\hline $2 E$ & 02 & - & BE & 0 \\
\hline & 02 & - & $\begin{array}{l}\mathrm{OE} \\
8 \mathrm{E}\end{array}$ & $\begin{array}{l}0 \\
0\end{array}$ \\
\hline & 02 & 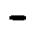 & $6 E$ & o \\
\hline $\mathrm{bE}$ & 02 & - & & \\
\hline & 02 & - & & \\
\hline
\end{tabular}

FREQ FREQ PERCENT

PERCENT

CUM FREQ FREO CUM

$0.0 \quad 0.0$

0.0

$\begin{array}{ll}0.0 & 0.0 \\ 0.0 & 0.0\end{array}$

$0.0 \quad 0.0$

$0.0 \quad 0.0$

$0.0 \quad 0.0$

$0.0 \quad 0.0$

$0.0 \quad 0.0$

$0.0 \quad 0.0$

$\begin{array}{ll}0.0 & 0.0 \\ 0.0 & 0.0\end{array}$

$3.23 \quad 3.23$

HISTOGRAM FOR COLUMN

12 BI PPM

1.0E $03 \times \times X$

$\begin{array}{ccccccc}N & L & H & B & T & G & \text { ANALYTICAL } \\ 30 & 0 & 0 & 0 & 0 & 0 & 1 \\ 96.77 & 0.0 & & & 0.0 & 0.0 & \end{array}$

MAXIMUM $=1.00000 E 03$

MINiMUM $=1.00000 E 03$

GEOMETRIC MEAN $=9.99999 \mathrm{E} 02$

GEOMETRIC DEVIATION $=9.99900 E 48$ 
FREQUENCY TABLE FOR COLUMN 13 (.CD PPM)

LIMITS FREQ FREG PERCENT PERCENT

ANALYTICAL

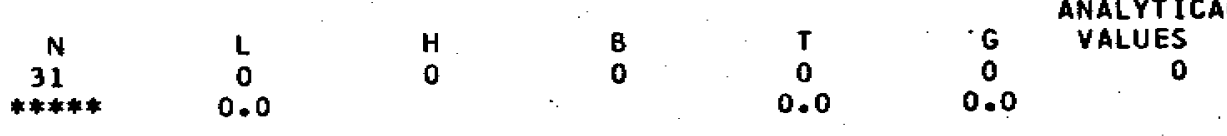

MAXIMUM $=-9.99900 E 48$

MINIMUM $=9.99900 E 48$

GEOMETRIC MEAN $=9.99900 E 48$

GEOMETRIC DEVIATION = $9.99900 E 4 \mathrm{~B}$ 
FREQUENCY TABLE FOR COLUMN

14, CO PPM,

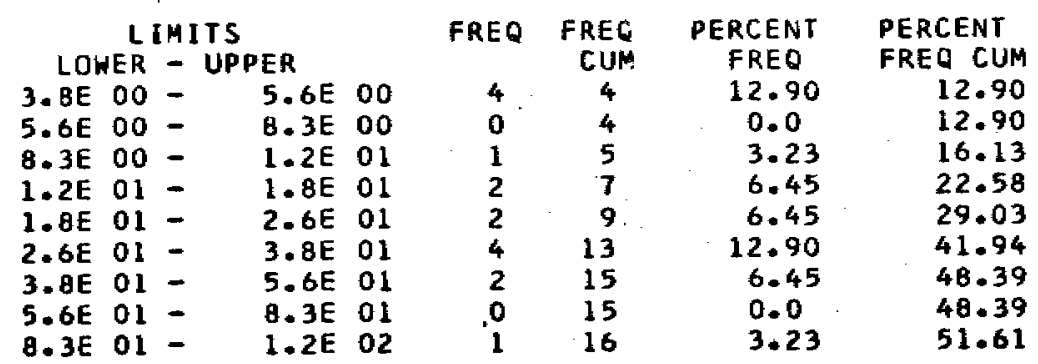

HISTOGRAM FOR COLUMN 14, CO PPM )

5. 0 E $00 \quad \times \times \times \times \times \times \times \times \times \times \times \times x$

7. OE 00

1. $0 \mathrm{E} 01 \times \times \times$

1.5E $01 \times \times \times \times \times x$

2.0E $01 \quad x \times x \times x \times$

3. $0 \mathrm{E} \quad 01 \quad \times \times \times \times \times \times \times \times \times \times \times \times \times$

实

5.0E $01 \times \times \times \times \times \times$

7.0E 01

1. OE $02 \times \times X$

$\begin{array}{ccccccc}\text { N } & & & & & \text { ANALYT ICAL } \\ 15 & L & H & 8 & T & G & \text { VALUES } \\ 48.39 & 0.0 & 0 & 0 & 0 & 0 & 16\end{array}$

MAXIMUM $=1.00000 E 02$

MINIMUH $=5.00000 E 00$

GEOMETRIC MEAN $=1.79280 E$ O1

GEOMETRIC DEVIATION $=2.54321$ EO 
FREQUENCY TABLE FOR COLUMN

15 ( CR PPM)

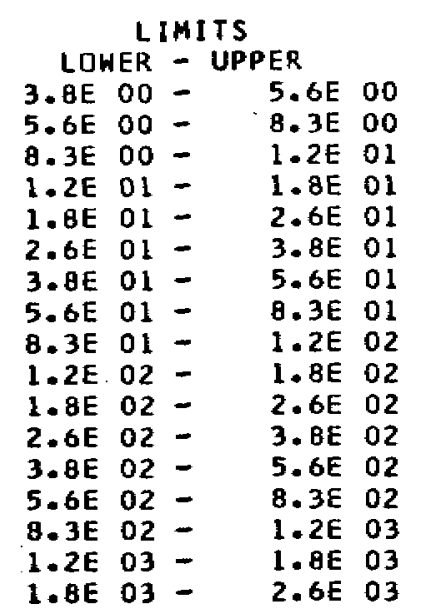

FREQ FREQ
CUMM

PERCENT

PERCENT

FREQ FREQ CUM

$\begin{array}{ll}0.0 & 0.0 \\ 0.0 & 0.0\end{array}$

$19.35 \quad 19.35$

$3.23 \quad 22.58$

$9.68 \quad 32.26$

$3.23 \quad 35.48$

3.23
$3.23 \quad 38.71$

$\begin{array}{ll}3.23 & 38.71 \\ 3.23 & 41.94\end{array}$

$3.23 \quad 45.16$

$3.23 \quad 48.39$

$3.23 \quad 51.61$

$0.0 \quad 51.61$

$0.0 \quad 51.61$

$0.0 \quad 51.61$

$0.0 \quad 51.61$

$0.0 \quad 51.61$

$\begin{array}{llllll}1.8 E & 03 & 0 & 16 & 0.0 & 51.61 \\ 2.6 E & 03 & 1 & 17 & 3.23 & 54.84\end{array}$

HISTOGRAM FOR COLUMN 15 ( CR PPM)

1. OE $01 \quad \times \times \times \times \times \times \times \times \times \times \times \times \times \times \times \times \times \times x$

1.5E $01 \times \times X$

2. OE $01 \times \times \times \times \times \times \times \times \times x$

N $3.0 \mathrm{E} 01 \mathrm{x} \times \mathrm{x}$

5. OE $01 \times \times x$

T. OE OI $\times \times x$

1. OE $02 \times \times X$

1.5E $02 \times x \times$

2. 0 E $02 \times \times x$

$3.0 \mathrm{E} 02$

5.OE 02

T. OE 02

1.OE 03

$1.5 \mathrm{E} 03$

2. OE $03 \times \times \times$

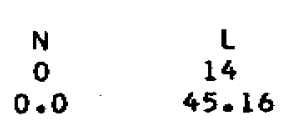


MINIMUM $=1.00000 E 01$

GEOMETRIC MEAN $=3.32861 \mathrm{E} O 1$

GEOMETRIC DEVIATION $=4.32273$ E 00 
FREQUENCY TABLE FOR COLUMN 16 i CU PPM )

\begin{tabular}{|c|c|c|c|c|c|c|c|c|}
\hline $\begin{array}{l}\angle O W \\
.8 E \\
.6 E \\
.3 E \\
.2 E\end{array}$ & $\begin{array}{l}\text { LI } \\
\text { JER } \\
00 \\
00 \\
00 \\
01 \\
01 \\
01 \\
01 \\
01 \\
01 \\
02 \\
02 \\
02 \\
02 \\
02 \\
02 \\
03 \\
03 \\
03 \\
03 \\
03 \\
03 \\
04\end{array}$ & 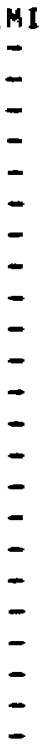 & $\begin{array}{l}\text { ER } \\
5.6 \mathrm{E} \\
8.3 \mathrm{E} \\
1.2 \mathrm{E} \\
1.8 \mathrm{E} \\
2.6 \mathrm{E} \\
3.8 \mathrm{E} \\
5.6 \mathrm{E} \\
8.3 \mathrm{E} \\
1.2 \mathrm{E} \\
1.8 \mathrm{E} \\
2.6 \mathrm{E} \\
3.8 \mathrm{E} \\
5.6 \mathrm{E} \\
8.3 \mathrm{E} \\
1.2 \mathrm{E} \\
1.8 \mathrm{E} \\
2.6 \mathrm{E} \\
3.8 \mathrm{E} \\
5.6 \mathrm{E} \\
8.3 \mathrm{E} \\
1.2 \mathrm{E} \\
1.8 \mathrm{E}\end{array}$ & $\begin{array}{l}00 \\
00 \\
01 \\
01 \\
01 \\
01 \\
01 \\
01 \\
02 \\
02 \\
02 \\
02 \\
02 \\
02 \\
03 \\
03 \\
03 \\
03 \\
03 \\
03 \\
04 \\
04\end{array}$ & $\begin{array}{c}\text { FREQ } \\
4 \\
4 \\
1 \\
4 \\
1 \\
2 \\
2 \\
0 \\
5 \\
1 \\
1 \\
0 \\
1 \\
0 \\
1 \\
0 \\
0 \\
0 \\
1 \\
0 \\
0 \\
0 \\
1\end{array}$ & $\begin{array}{c}\text { FREQ } \\
\text { CUM } \\
4 \\
5 \\
9 \\
20 \\
12 \\
14 \\
14 \\
19 \\
20 \\
21 \\
21 \\
22 \\
22 \\
23 \\
23 \\
23 \\
23 \\
24 \\
24 \\
24 \\
24 \\
25\end{array}$ & $\begin{array}{c}\text { PERCENT } \\
\text { FREQ } \\
12.90 \\
3.23 \\
12.90 \\
3.23 \\
6.45 \\
6.45 \\
0.0 \\
16.13 \\
3.23 \\
3.23 \\
0.0 \\
3.23 \\
0.0 \\
3.23 \\
0.0 \\
0.0 \\
0.0 \\
3.23 \\
0.0 \\
0.0 \\
0.0 \\
3.23\end{array}$ & $\begin{array}{l}\text { PERCENT } \\
\text { FREQ CUM } \\
12.90 \\
16.13 \\
29.03 \\
32.26 \\
38.71\end{array}$ \\
\hline
\end{tabular}

HISTOGRAM FOR COLUMN 16 ( CU PPM)

5. OE OO $\times \times \times \times \times \times \times \times \times \times \times \times x$

7. OE OO $X X X$

1. OE $01 \times \times \times \times \times \times \times \times \times \times \times \times \times$

1.5E $01 \times \times x$

2. OE $01 \times \times \times \times \times \times$

3.0E $01 \times \times \times \times \times \times$

5.0E 01

7.0E $01 \times \times \times \times \times \times \times \times \times \times \times \times \times \times \times \times$

1. OE $02 \times \times \times$

1.5E $02 \times \times \times$

2. OE 02

3. OE $02 \times X X$

5. OE 02

7. OE $02 \times \times X$

1. OE 03

1.5E 03 
2.OE 03

3. OE $03 \times \times x$

5.0E 03

7. OE 03

1. OE 04

1.5E $04 \times \times X$

$\begin{array}{cc}N & L \\ 0 & 6 \\ 0.0 & 19.35\end{array}$

$H$
0

$\begin{array}{cc}8 & T \\ 0 & 0 \\ & 0.0\end{array}$

$\begin{array}{cc} & \text { ANALYTICAL } \\ 6 & \text { VALUES } \\ 0 & 25 \\ 0.0 & \end{array}$

MAXIMUM $=1.50010 E 04$

MINIMUM $=5.00000 E 00$

GEOMETRIC MEAN $=4.26589 E$ OI

GEOMETRIC DEVIATION $=7.71448 E 00$

$\mathfrak{Ð}$ 


\section{FREQUENCY TABLE FOR COLUMN}

17 (LA PPM J

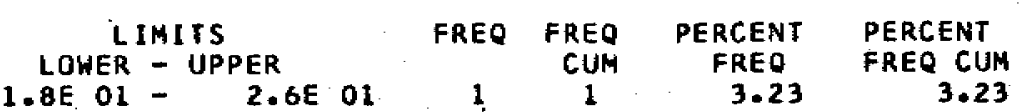

HISTOGRAM FOR COLUMN 17 ( LA PPM I

2. OE $01 \times \times \times$

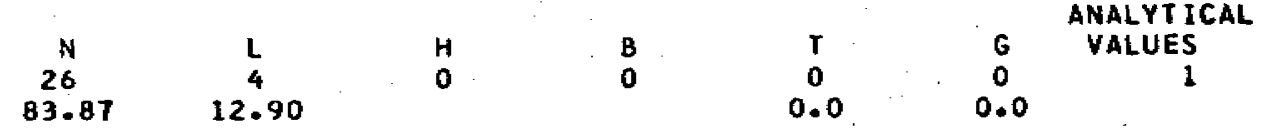

MAXIMUM $=2.00000 E 01$

MINIMUM $=2.00000 E 01$

GEOMETRIC MEAN $=1.99999 E 01$

GEOMETRIC DEVIATION $=9.99900 E 48$ 
FREQUENCY TABLE FOR COLUMN 18 I MO PPM I

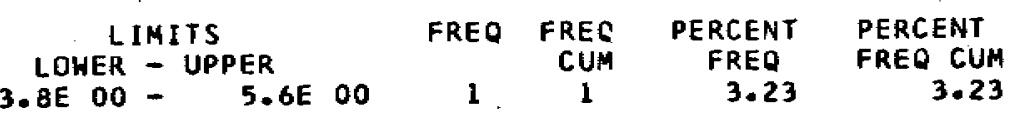

HISTOGRAM FOR COLUMN IB 1 MO PPM

5. OE OO XXX

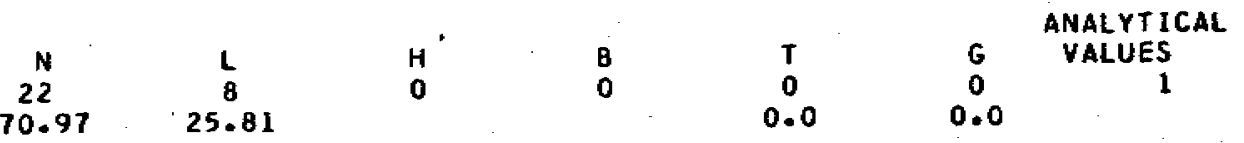

MAXIMUM $=5,00000 E 00$

MINIMUM $=5.00000 E 00$

GEOMETRIC MEAN $=5.00000 E$ DO

GEOMETRIC DEVIATION $=9.99900$ E 48 
FREQUENCY TABLE FOR COLUMN

19 ( NB PPM)

LIHI IS
LOHER - UPPER

FREQ FREQ PERCENT

CUM FREQ

PERCENT

FREQ CUM
25.81

6.3E $00-1.2 E 01$

( NB PPM )

HISTOGRAM FOR COLUMN 19 ( NB PPM )

1.0E $01 \times \times \times \times \times \times \times \times \times \times \times \times \times \times \times \times \times \times \times \times \times \times \times \times \times x$

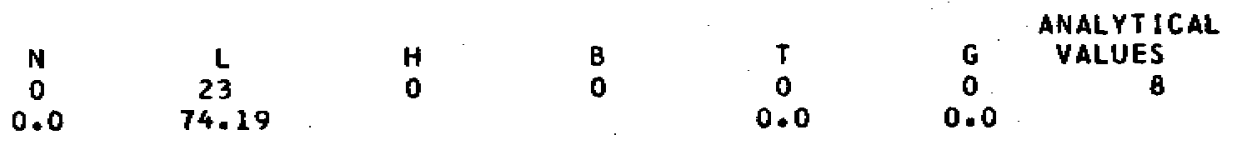

MAXIMUM $=1.00000 E 01$

MINIMUH $=1.00000 E$ OL

GEOMETRIC MEAN $=9.99997 E 00$

GEOMETRIC DEVIATION $=1.00208 E 00$ 
FREQUENCY TABLE FOR COLUMN $20 \mid \mathrm{NI}$ PPM

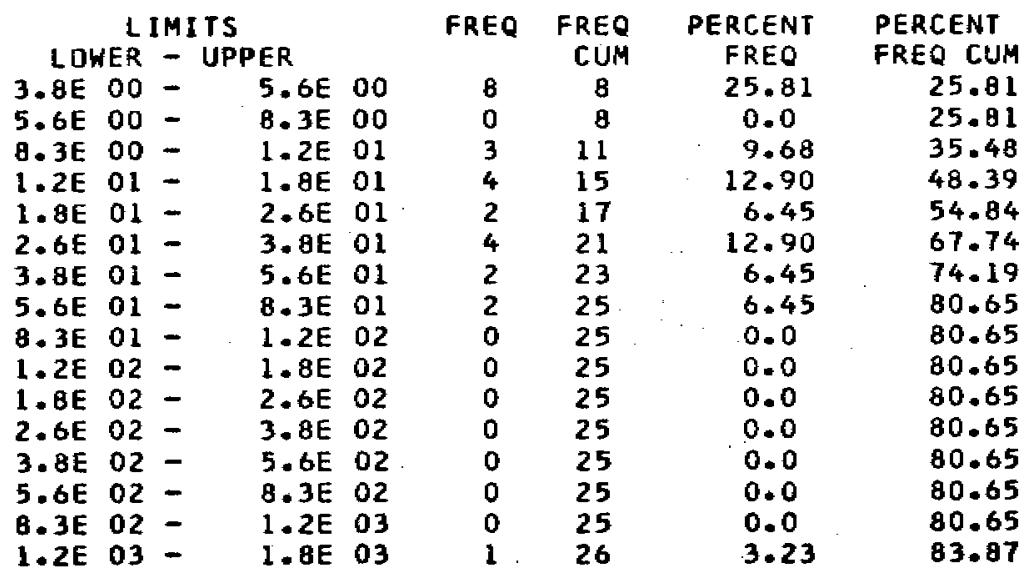

HISTOGRAM FOR CDLUMN 20 I NI PPM

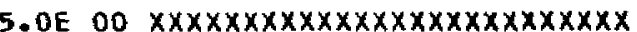

7. OE OO

1. OE $01 \times X \times X \times X \times X \times X X$

1. $5 \mathrm{E} 01 \times \times \times \times \times \times \times \times \times \times \times \times \times$

2. OE $01 \times \times \times \times \times x$

3. OE $01 \quad x \times x \times x \times x \times x \times x \times x$

5. OE $01 \quad \times \times \times \times \times x$

7.0E $01 \times \times \times \times \times \times$

1. OE 02

1.5E 02

$2.0 E \quad 02$

$3.0 E \quad 02$

5.OE O2

7.0E 02

1. OE 03

$1.5 E 03 \times \times X$

$\begin{array}{ccccccc}N & \text { L } & H & \text { B } & T & G & \text { ANALYTICAL } \\ 0 & 5 & 0 & 0 & 0 & 0 & 26 \\ 0.0 & 16.13 & & & 0.0 & 0.0 & \end{array}$


MINIMUM $=5.00000 E 00$

GEOMETRIC MEAN $=1.71195 E$ O1

GEOMETRIC DEVIATIDN $=3.57839$ OO 
FREQUENCY TABLE FOR COLUMN 21 ( PB PPH ,

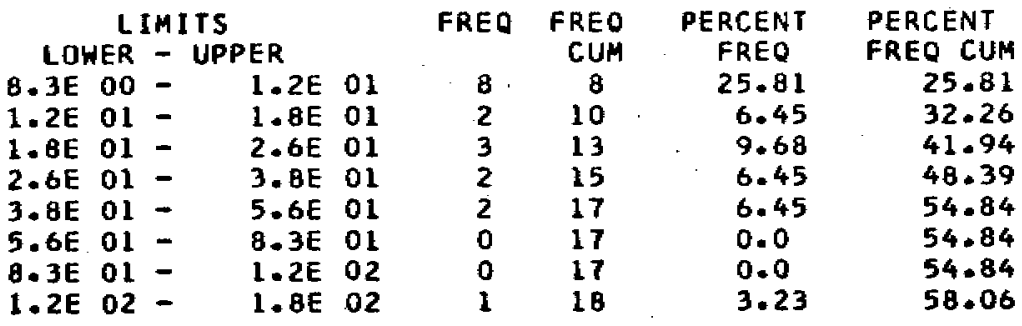

histogram for COLUmN 21 I PB PPM I

1.0E $01 \quad \times \times \times \times \times \times \times \times \times \times \times \times \times \times \times \times \times \times \times \times \times \times \times \times \times x$

1.5E $01 \quad x \times \times \times \times X$

2. 0 OE $01 \times \times \times \times \times \times \times \times \times \times$

3. 0 E $01 \times \times \times \times \times x$

5. OE $01 \quad \times \times \times \times \times x$

थ $\quad$ T.OE 01

1. OE 02

1. $5 E 02 \times \times x$

$\begin{array}{ccccccc}N & & & & & & \text { ANALYTICAL } \\ 9 & L & H & B & T & 0 & \text { VALUES } \\ 29.03 & 12.90 & 0 & 0 & 0 & 0 & 18\end{array}$

MAXIMUM $=1.50000 \mathrm{E} 02$

MINIMUM $=1.00000 E$ OI

GEOMETRIC MEAN $=1.04394 \mathrm{E}$ O1

GEOMETRIC DEVIATION $=2.14981 \mathrm{E}$ OO 
frequenCy table for COLUMN 22 I SB PPM 1

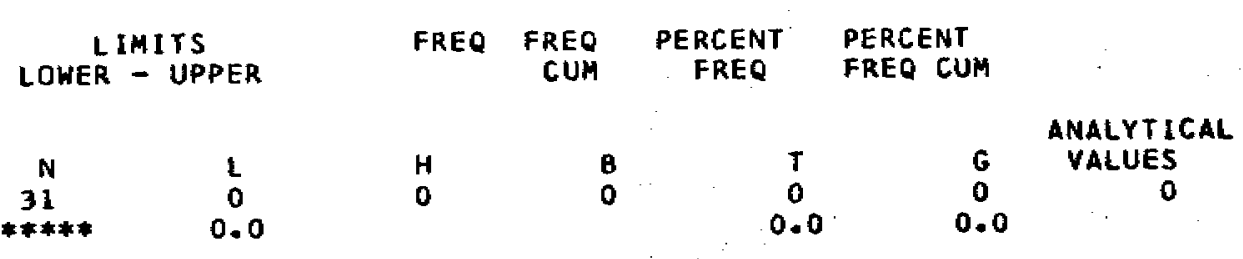

MAXIMUM $=-9.99900 E 48$

MINIMUM $=9.99900 E 48$

GEOMETRIC MEAN $=9.99900 E 48$

GEOMETRIC DEVIATION $=9.99900 E 48$ 
FREQUENCY TABLE FOR COLUMN

23 ISC PPM ;

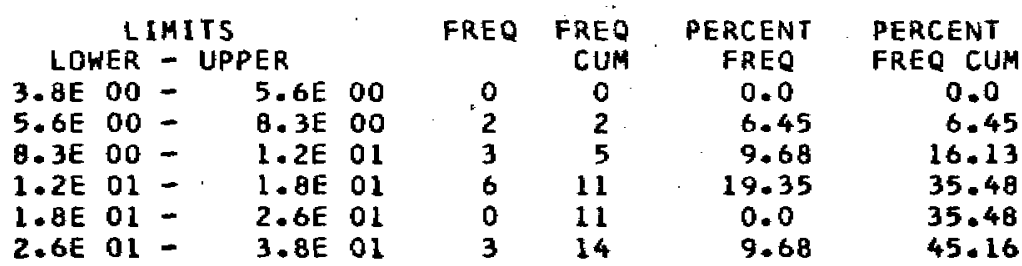

HISTOGRAM FOR CDLUMN 23 ( SC PPM ,

7. OE $00 \quad \times \times \times \times \times \times$

\section{OE Ol $\times \times \times \times \times \times \times \times \times x$}

\subsection{E O1 $x \times x \times x \times x \times x \times x \times x \times x \times x \times x$}

2.0E 01

3. 0 E $01 \times \times \times \times \times \times \times \times \times x$

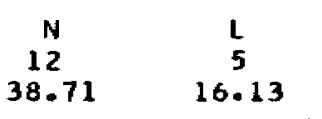

$T$
0
0.0

$\begin{array}{cc} & \text { ANALYTICAL } \\ 6 & \text { VALUES } \\ 0 & 14 \\ 0.0 & \end{array}$

W MAXIMUM $=3.00000 \mathrm{E}$ OL

MINIMUM $=7.00000 E$ OO

GEOMETRIC MEAN $=1.43079 E$ OL

GEOMETRIC DEVIATION $=1.62509$ E 00 
FREQUENCY TABLE FOR COLUMN 24 ( SN PPM )

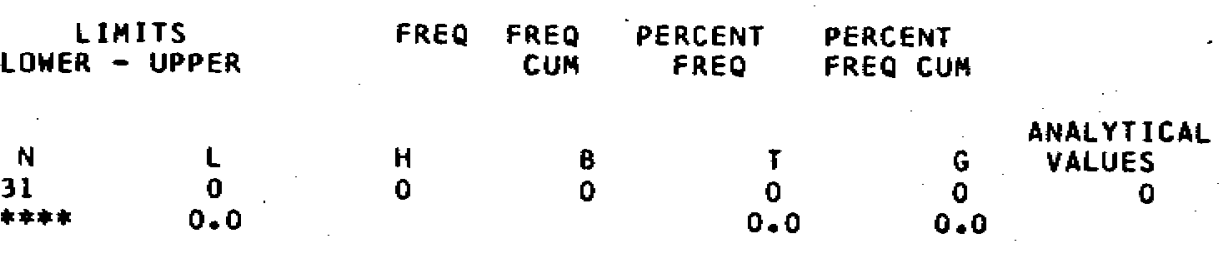

MAXIMUM $=-9.99900 E 48$

MINIMUM $=9.99900 E 48$

GEOMETRIC MEAN $=9.99900 E 48$

GEAMETRIC DEVIATION $=9.99900 E 48$ 
FREQUENCY TABLE FOR COLUMN

24 SN PPM

LIHITS
LOWER - UPPER

FREQ FREQ PERCENT
CUM FREO

PERCENT

FREQ FREQ CUM

$\begin{array}{cc}N & L \\ 31 & 0\end{array}$

$H$
0

B
0

$T$
0
0.0

ANALYTICAL

VALUES

MAXIMUM $=-9.99900 E 48$

MINIMUH $=9.99900 E 48$

GEOMETRIC MEAN $=9.99900 E 48$

GEOMETRIC DEVIATION $=9.99900 E 48$ 
FREQUENCY TABLE FOR COLUMN 25 I SR PPM ।

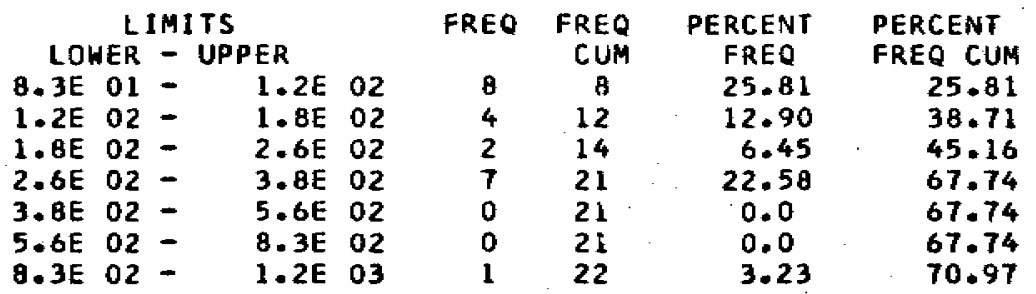

HISTOGRAM FOR COLUMN 25 ( SR PPM)

1.0E $02 \quad x \times \times \times \times \times \times \times \times \times \times \times \times \times \times \times \times \times \times \times \times \times \times \times \times x$

1.5E $02 \quad x \times x \times x \times x \times x \times x \times x$

2. OE $02 \times \times \times \times \times \times$

3. 0 E $02 \quad x \times x \times x \times x \times x \times x \times x \times x \times x \times x \times x \times x$

5. OE 02

ثี

7. OE 02

1. OE $03 \times \times x$

$\begin{array}{ccccccc}N & t & H & B & T & G & \begin{array}{c}\text { ANALYTICAL } \\ \text { VALUES }\end{array} \\ 0.0 & 9 & 0 & 0 & 0 & 0 & 22\end{array}$

MAXIMUM $=1.00000 E 03$

MINIMUM $=1.00000 E 02$

GEOMETRIC MEAN $=1.80570 E .02$

GEOMETRIC DEVIATION $=1.83130 E$ OO 
FREQUENCY TABLE FOR COLUMN $26, V$ PPM ,

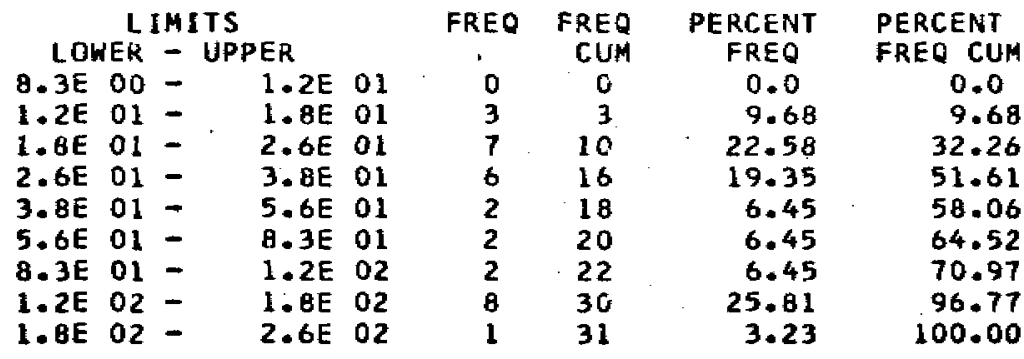

HISTOGRAM FOR COLUMN 261 Y PPH,

1.5E $01 \times \times \times \times \times \times \times \times \times \times$

2. OE $01 \quad x \times x \times x \times x \times x \times x \times x \times x \times x \times x \times x \times x$

3. OE $01 \quad x \times x \times x \times x \times x \times x \times x \times x \times x \times x$

5. 0 E $01 \times \times \times \times \times \times$

7. OE $01 \times \times \times \times \times \times$

1.0E. $02 \times \times \times \times \times x$

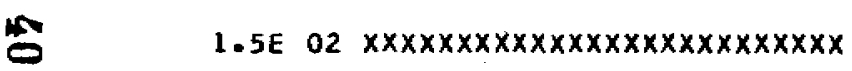

2.0E $02 \times \times X$

$\begin{array}{ccccccc}N & L & H & B & T & 6 & \text { ANALYTICAL } \\ 0 & 0 & 0 & 0 & 0 & 0 & 31 \\ 0.0 & 0.0 & & & 0.0 & 0.0 & \end{array}$

MAXIMUH $=2.00000 E 02$

MINIMUM $=1.50000 \mathrm{E} 01$

GEOMETRIC MEAN $=4.86369 E$ OI

GEOMETRIC DEVIATION $\approx 2.47025 E 00$ 
FREQUENCY TABLE FOR COLUMN 27 , $\mathrm{WPM}$,

LIMITS FREQ FREQ PERCENT PERCENT LOWER - UPPER CUM PREQ FREQ CUM

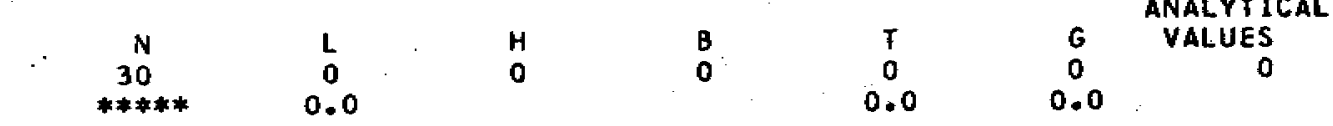

MAXIMUM $=-9.99900 E 48$

MINIMUM $=9.99900 \mathrm{E} 48$

GEOMETRIC MEAN $=9.99900 E 48$

GEOHETRIC DEVIATION *9.99900E 48 
FREQUENCY TABLE FOR COLUMN

$28 \mid Y$ PPM

\begin{tabular}{|c|c|c|c|c|c|c|c|}
\hline 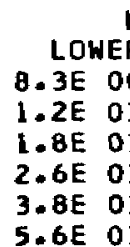 & 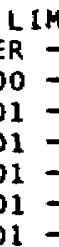 & 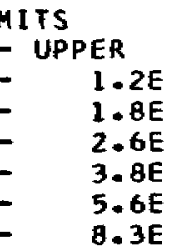 & $\begin{array}{l}01 \\
01 \\
01 \\
01 \\
01 \\
01\end{array}$ & $\begin{array}{l}9 \\
8 \\
2 \\
1 \\
0 \\
1\end{array}$ & $\begin{array}{l}\text { FREQ } \\
\text { CLH } \\
9 \\
17 \\
19 \\
20 \\
20 \\
21\end{array}$ & $\begin{array}{c}\text { PERCENT } \\
\text { FREQ } \\
29.03 \\
25.81 \\
6.45 \\
3.23 \\
0.0 \\
3.23\end{array}$ & $\begin{array}{r}\text { PERCENT } \\
\text { FREQ CUM } \\
29.03 \\
54.84 \\
61.29 \\
64.52 \\
64.52 \\
67.74\end{array}$ \\
\hline
\end{tabular}

HISTOGRAM FOR COLUMN $28, Y$ PPH

1. OE $01 \quad x \times x \times x \times x \times x \times x \times x \times x \times x \times x \times x \times x \times x \times x \times x$

\subsection{E $01 \quad \times \times \times \times \times \times \times \times \times \times \times \times \times \times \times \times \times \times \times \times \times \times \times \times \times x$}

2.0E $01 \times \times \times \times \times x$

3.0E $01 \times X X$

5.OE O1

7. OE $01 \times \times \times$

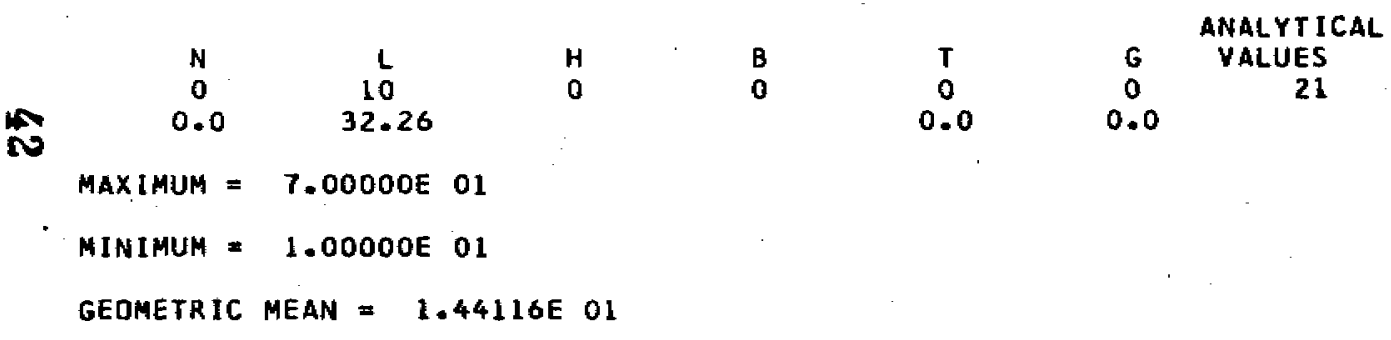

GEOMETRIC DEVIATION $=1.60400 E 00$ 
FREQUENCY TABLE FOR COLUMN 29 ( $2 N$ PPM I

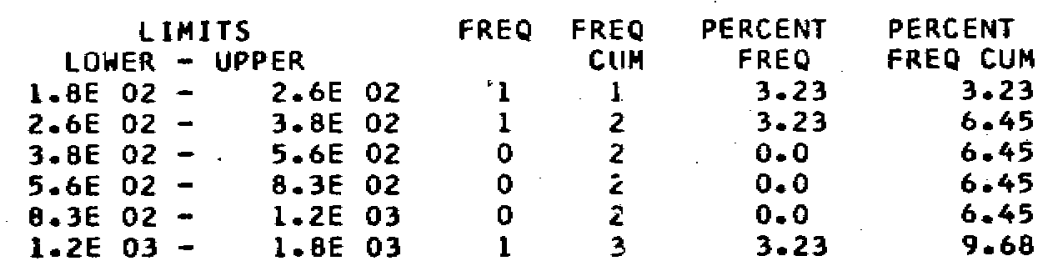

HISTOGRAM FOR COLUMN 29 ( 2N PPM)

2. OE $02 \times \times x$

$3.0 E 02 \times \times X$

$5.0 \mathrm{E} \quad 02$

7.OE 02

$1.0 E \quad 03$

1.5E $03 \times \times x$

\begin{tabular}{|c|c|c|c|c|c|c|c|}
\hline 4 & $\begin{array}{c}N \\
22 \\
70.97\end{array}$ & $\begin{array}{c}L \\
6 \\
19.35\end{array}$ & $\begin{array}{l}H \\
0\end{array}$ & $\begin{array}{l}B \\
0\end{array}$ & $\begin{array}{c}T \\
0 \\
0.0\end{array}$ & $\begin{array}{c}6 \\
0 \\
0.0\end{array}$ & $\begin{array}{c}\text { ANALYT ICAL } \\
\text { VALUES } \\
3\end{array}$ \\
\hline
\end{tabular}

MAXIMUM $=1.50000 \mathrm{E} 03$

MINIMUM $=2.00000 \mathrm{E} 02$

GEOMETRIC MEAN $=4.48139 E$ O2

GEOMETRIC DEVIATION = 2.90291 OO 
FREQUENCY TABLE FOR COLUMN 30 ( ZR PPM-)

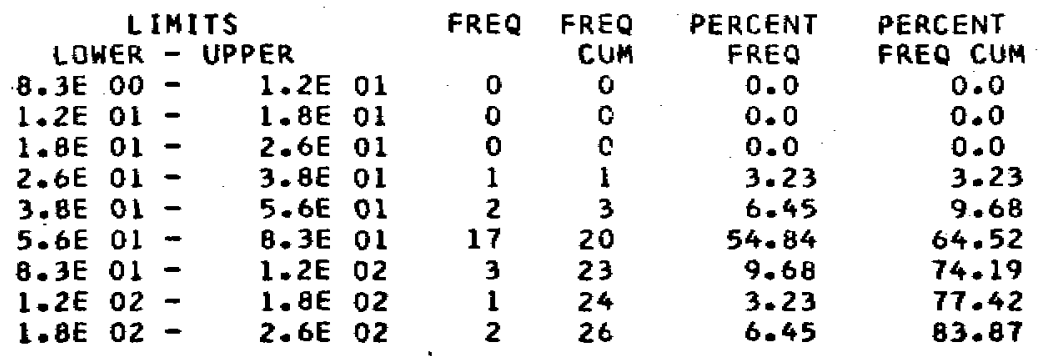

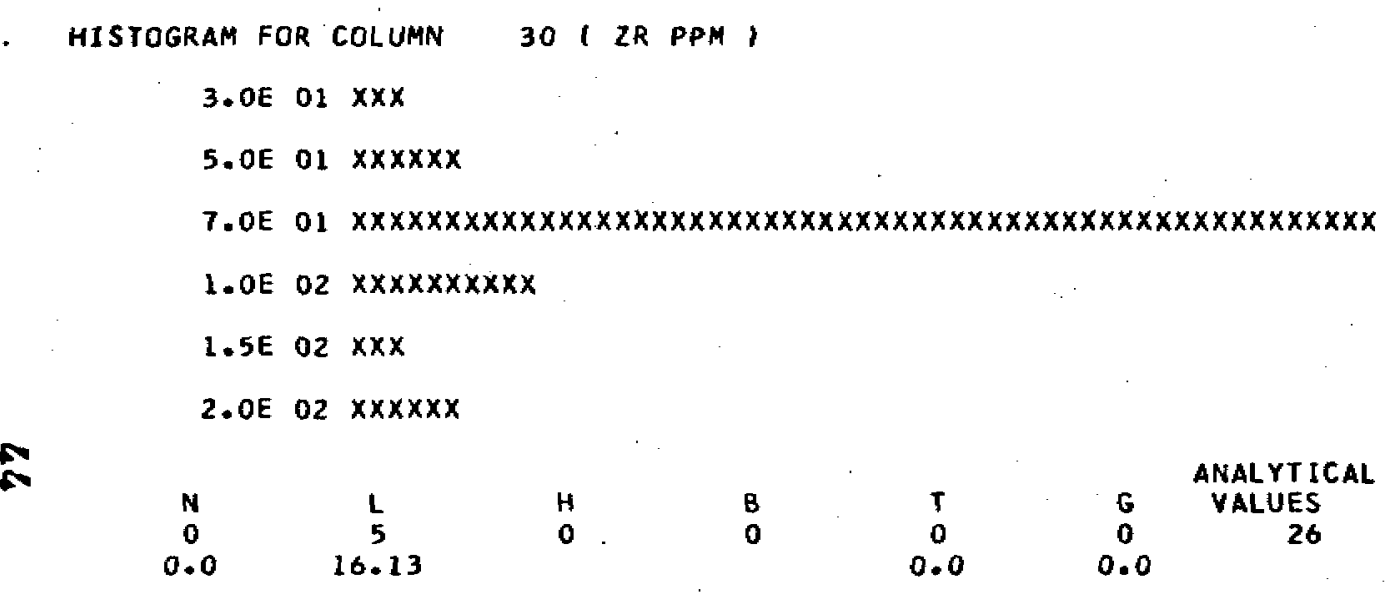

MAXIMUM $=2,00000 \mathrm{E} 02$

MINIMUM $=3.00000 E 01$

GEOHETRIC MEAN $=7.68025 E 01$

GEOMETRIC DEVIATION $=1.48346 E$ OO 
FREQUENCY TABLE FOR COLUMN 31 ( AU PPM )

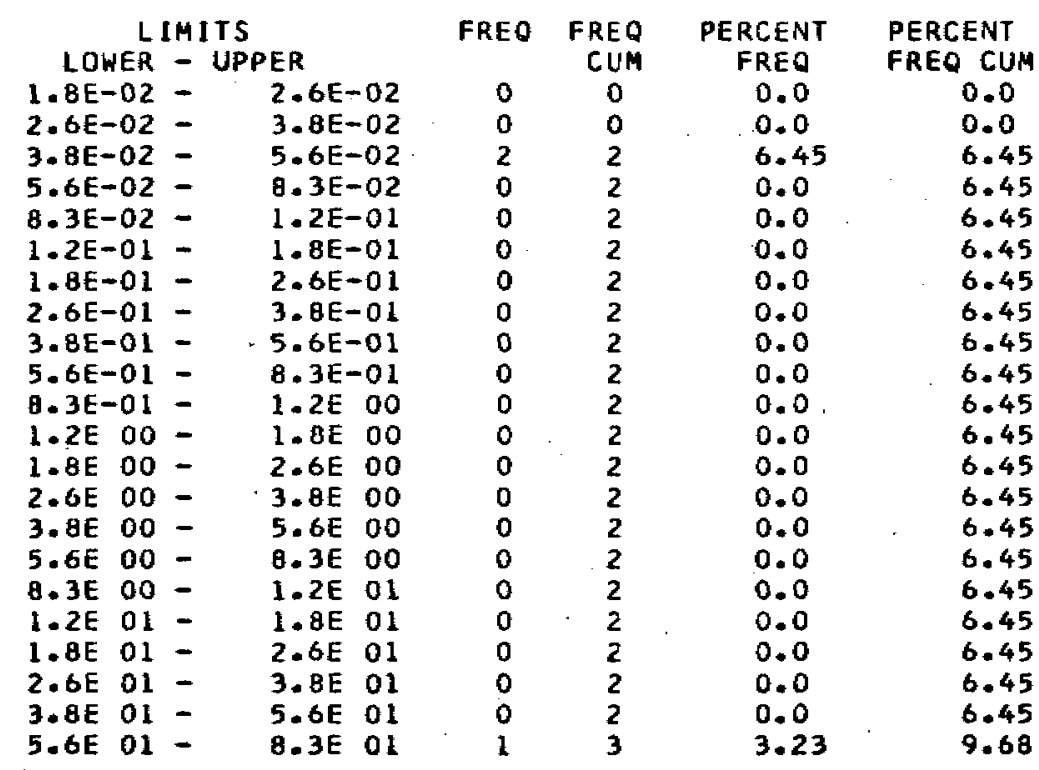

E HISTOGRAM far COLUMN 311 AU PPM ।

$$
\begin{aligned}
& \text { 5.0E-02 } x \times X \times X X \\
& 7.0 E-02 \\
& 1.0 E-01 \\
& 1.5 E-01 \\
& 2.0 E-01 \\
& 3.0 E-01 \\
& 5.0 E-01 \\
& \text { 7.OE-01 } \\
& \text { L.OE } 00 \\
& 1.5 E 00 \\
& 2.0 E 00 \\
& 3.0 E 00 \\
& 5.0 E 00 \\
& 7.0 E 00 \\
& 1.0 E 01
\end{aligned}
$$

CUM

0.45

6.45

6.45

6.45

6.45

6.45

6.45

9.68

1.5E O1 
2. 0 E 01

3.0E 01

5.0E OL

7. OE O1 $\mathrm{xXX}$

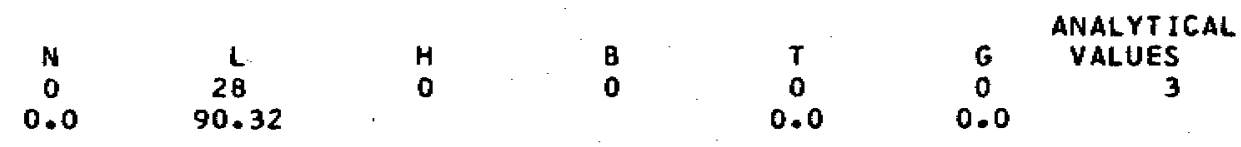

MAXIMUM $=6.00000 E 01$

MINIMUM $=4.00000 E-02$

GEOMETRIC MEAN $=4.57886 E-01$

GEOMETRIC DEVIATION $=6.81891 E$ O1 


\begin{tabular}{lr} 
ELEMENT & N \\
FE PCT & 0 \\
MG PCT & 0 \\
CA PCT & 0 \\
TI PCT & 0 \\
MN PPM & 0 \\
AG PPM & 0 \\
AS PPM & 18 \\
AU PPM & 31 \\
B PPM & 31 \\
BA PPM & 16 \\
BE PPM & 0 \\
BI PPM & 17 \\
CD PPM & 30 \\
CD PPM & 31 \\
CR PPM & 15 \\
CU PPM & 0 \\
LA PPM & 0 \\
MO PPM & 26 \\
NB PPM & 22 \\
NI PPM & 0 \\
PB PPM & 0 \\
SB PPM & 9 \\
SC PPM & 31 \\
SN PPM & 12 \\
SR PPM & 31 \\
V PPM & 0 \\
H PPM & 0 \\
Y PPM & 30 \\
ZN PPM & 0 \\
ZR PPM & 22 \\
AU PPM & 0 \\
\hline &
\end{tabular}

$\begin{array}{rl}\mathrm{L} & H \\ 1 & \\ 1 & 0 \\ 6 & 0 \\ 1 & 0 \\ 1 & 0 \\ 5 & 0 \\ 0 & 0 \\ 0 & 0 \\ 8 & 0 \\ 3 & 0 \\ 9 & 0 \\ 0 & 0 \\ 0 & 0 \\ 0 & 0 \\ 14 & 0 \\ 6 & 0 \\ 4 & 0 \\ 8 & 0 \\ 23 & 0 \\ 5 & 0 \\ 4 & 0 \\ 0 & 0 \\ 5 & 0 \\ 0 & 0 \\ 9 & 0 \\ 0 & 0 \\ 0 & 0 \\ 10 & 0 \\ 6 & 0 \\ 5 & 0 \\ 28 & 0\end{array}$

$\begin{array}{lll}H & B & T \\ 0 & 0 & 0 \\ 0 & 0 & 0 \\ 0 & 0 & 0 \\ 0 & 0 & 0 \\ 0 & 0 & 0 \\ 0 & 0 & 0 \\ 0 & 0 & 0 \\ 0 & 0 & 0 \\ 0 & 0 & 0 \\ 0 & 0 & 0 \\ 0 & 0 & 0 \\ 0 & 0 & 0 \\ 0 & 0 & 0 \\ 0 & 0 & 0 \\ 0 & 0 & 0 \\ 0 & 0 & 0 \\ 0 & 0 & 0 \\ 0 & 0 & 0 \\ 0 & 0 & 0 \\ 0 & 0 & 0 \\ 0 & 0 & 0 \\ 0 & 0 & 0 \\ 0 & 0 & 0 \\ 0 & 0 & 0 \\ 0 & 0 & 0 \\ 0 & 0 & 0 \\ 0 & 0 & 0 \\ 0 & 0 & 0 \\ 0 & 0 & 0 \\ 0 & 0 & 0 \\ 0 & 0 & 0 \\ 0 & & 0\end{array}$

ANALYTICAL

VALUES

\begin{tabular}{|c|c|c|c|}
\hline \multirow{2}{*}{\multicolumn{2}{|c|}{ FI EMFNT }} & GEOMETRIC & GEOME TRIC \\
\hline & & MEAN & DE VIATION \\
\hline FE & PCT & 1.978743 & 3.53 \\
\hline MG & PCT & 0.578295 & 3.88 \\
\hline $\mathbf{C A}$ & PCT & 0.564127 & 8.10 \\
\hline TI & PCT & 0.131897 & 3.44 \\
\hline MN & PPM & 224.465714 & 4.05 \\
\hline AG & PPM & 0.043489 & 28.15 \\
\hline AS & PPM & ******** & 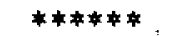 \\
\hline AU & PPM & $* * * * * * * *$ & ****** \\
\hline B & PPM & 4.255919 & 2.42 \\
\hline$B A$ & PPM & 317.109619 & 3.80 \\
\hline$B E$ & PPM & 0.375585 & $2 \cdot 16$ \\
\hline B I & PPM & $\# * * * * * * *$ & $\neq * * * *$ \\
\hline$C D$ & PPM & $* * * * * * * *$ & $* * * * *$ \\
\hline Co & PPM & 4.447355 & 5.69 \\
\hline CR & PPM & 5.462089 & $11.7,0$ \\
\hline $\mathrm{cu}$ & PPM & 20.170502 & 11.53 \\
\hline LA & PPM & $* * * * * * * \neq$ & $\star * * * * *$ \\
\hline MO & PPM & $* * * * * * * *$ & $* * * * * *$ \\
\hline NB & PPM & 7.053754 & 1.30 \\
\hline NI & PPM & 11.704411 & $4 \cdot 40$ \\
\hline PB & PPM & 9.395220 & 2.89 \\
\hline
\end{tabular}

\section{REMARKS}

1 NOT DETECTED, LESS THAN, or tRaCe VALUES. 1 NOT DETECTED, LESS THAN, OR TRACE VALUES. 6 NOT DETECTED, LESS THAN, OR TRACE VALUES. 1 NOT DETECIED, LESS THAN, OR TRACE VALUES. 1 NOT DETECTED, LESS IHAN, OR TRACE VALUES. 23 NOT DETECTED, LESS THAN, OR TRACE VALUES. 31 NOT DETECTED, LESS THAN, OR TRACE VALUES. 31 NOT DETECTED, LESS THAN, OR TRACE VALUES. 24 NOT DETECTED, LESS THAN, OR TRACE VALUES. 3 NOT DETECTED, LESS THAN, OR TRACE VALUES. 26 NOT DETECTED, LESS THAN, OR TRACE VALUES. 30 NOT DETECTED, LESS THAN, OR TRACE VALUES. 31 NOT DETECTED, LESS THAN, OR TRACE VALUES. 15 NOT DETECTED, LESS THAN, OR TRACE VALUES. 14 NOT DETECTED, LESS THAN, OR TRACE VALUES. 6 NOT DETECTED, LESS THAN, OR TRACE VALUES. 30 NOT DETECTED, LESS THAN, OR TRACE VALUES. 30 NOT DETECTED, LESS THAN, OR TRACE VALUES. 23 NOT DETECTED; LESS THAN, OR TRACE VALUES. 5 NOT DETECTED, LESS THAN, DR TRACE VALUES. 13 NOT DETECTED, LESS THAN, OR TRACE VALUES
30 REPORTED VALUES. 30 REPORTED VALUES. 25 REPORTED VALUES. 30 REPORTED VALUES. 30 REPORTED VALUES. 8 REPORTED VALUES.

O REPORTED VALUES. NO COMPUTATIONS. O REPORTED VALUES. ND COMPUTATIONS. 7 REPORTED VALUES.

28 REPORTED VALUES.

5 REPORTED VALUES.

1 REPORTED VALUES. NO CDMPUTATIONS. D REPORTED VALUES. NO COMPUTATIONS. 16 REPORTED VALUES.

17 REPORTED VALUES.

25 REPORTED VALUES.

1 REPORTED VALUES. NO COMPUTATIONS.

1 REPORTED VALUES. NO COMPUTATIONS.

8 REPORTED VALUES.

26 REPORTEO VALUES.

18 REPORTEO VALUES 


$\begin{array}{llcr}\text { SB PPM } & * * * * * * * & * * * * * * \\ \text { SC PPM } & 3.706535 & 4.14 \\ \text { SN PPM } & * * * * * * * * & * * * * * * \\ \text { SR PPM } & 123.545486 & 2.26 \\ \text { V PPM } & 48.636841 & 2.47 \\ \text { W PPM } & * * * * * * * & * * * * * \\ Y \text { PPM } & 10.484584 & 1.88 \\ \text { ZN PPM } & * * * * * * * & * * * * * * \\ \text { ZR PPH } & 49.934845 & 2.88 \\ \text { AU PPM } & * * * * * * * * & * * * * * *\end{array}$

31 NOT DETECTED, LESS THAN, OR TRACE VALUES. 17 NOT DETECTEO, LESS THAN, OR TRACE VALUES. 31 NOT DETECTED, LESS THAN, OR TRACE VALUES. 9 NOT DETECTED, LESS THAN, OR TRACE VALUES. 31 SAMPLES AND 31 ANALYTICAL VALUES.

30 NOT DETECTED, LESS THAN, OR TRACE VALUES.

10 NOT DETECTED, LESS THAN, OR TRACE VALUES

10 NOT DETECTED, LESS THAN, OR TRACE VALUES

5 NOT DETECTED, LESS THAN, OR TRACE VALUES.

5 NOT DETECTED, LESS THAN, OR TRACE VALUES.

28 NOT DETECTED, LESS THAN, DR TRACE VALUES.
O REPOR TED VALUES. NO COMPUTATIONS. 14 REPDRTED VALUES.

o reported values. no computations. 22 REPORTED VALUES.

O. REPORTEO VALUES. NO COMPUTATIONS. 21 REPORTEO VALUES.

3 REPORTED VALUES. NO COMPUTATIONS. 26 REPORTED VALUES.

3 REPORTED VALUES. NO COMPUTATIONS. 
TABLE 3. SUMDUM C-4 STREAM SEDIMENTS

\begin{tabular}{|c|c|c|c|c|c|c|c|c|}
\hline $\begin{array}{l}\text { Muppe } \\
14 \\
20\end{array}$ & $\begin{array}{l}\text { SAMPLE } \\
\text { AKDO29 } \\
\text { AKD797 }\end{array}$ & $\begin{array}{c}\text { FE PCT } \\
15.0000 \\
5.0000\end{array}$ & $\begin{array}{l}\text { MG PCT } \\
2.0000 \\
1.5000\end{array}$ & $\begin{array}{l}\text { CA PCT } \\
2.0000 \\
1.5000\end{array}$ & $\begin{array}{l}\text { TI PCT } \\
0.5000 \\
0.3000\end{array}$ & $\begin{array}{c}\text { MN PPM } \\
700.0000 \\
700.0000\end{array}$ & $\begin{array}{r}\text { AG } \\
0.0 \\
0.0\end{array}$ & $\begin{array}{r}P P M \\
N \\
N\end{array}$ \\
\hline 21 & AKD799 & 5.0000 & 2.0000 & 2.0000 & 0.5000 & 1000.0000 & 0.0 & $\mathbf{N}$ \\
\hline 22 & AKD796 & 5.0000 & 2.0000 & 2.0000 & 0.7000 & 1000.0000 & 0.0 & $\mathbf{N}$ \\
\hline 23 & AKDB 30 & 5.0000 & 2.0000 & 1.5000 & 0.5000 & 700.0000 & 0.0 & $\mathbf{N}$ \\
\hline 23 & AKD 31 & 3.0000 & 1.5000 & 2.0000 & 0.3000 & 700.0000 & 0.0 & $\mathbf{N}$ \\
\hline 24 & AK0832 & 3.0000 & 1.5000 & 1.5000 & 0.3000 & 700.0000 & 0.0 & $\mathbf{N}$ \\
\hline 25 & AKD 800 & 5.0000 & 3.0000 & 2.0000 & 0.5000 & 1000.0000 & 0.0 & $\mathbf{N}$ \\
\hline 26 & AKD801 & 3.0000 & 2.0000 & 1.5000 & 0.3000 & 500.0000 & 0.0 & N \\
\hline 27 & AKDBO2 & 3.0000 & 1.5000 & 1.5000 & 0.3000 & 700.0000 & 0.0 & $\mathbb{N}$ \\
\hline 28 & $A K D B O 3$ & 3.0000 & 1.5000 & 1.5000 & 0.2000 & 300.0000 & 0.0 & $\mathbf{N}$ \\
\hline $2 \varphi$ & AKDBO4 & 3.0000 & 1.5000 & 1.0000 & 0.3000 & 500.0000 & 0.0 & $\mathbf{N}$ \\
\hline 30 & AKD805 & 3.0000 & 1.5000 & 1.5000 & 0.3000 & 700.0000 & 0.0 & in \\
\hline 31 & AKDBO6 & 3.0000 & 1.5000 & 1.5000 & 0.3000 & 300.0000 & 0.0 & $\mathbf{N}$ \\
\hline 32 & AKDBO7 & 5.0000 & 3.0000 & 2.0000 & 0.5000 & 700.0000 & 0.0 & $\mathbf{N}$ \\
\hline 37 & AKD824 & 10.0000 & 3.0000 & 1.5000 & 0.7000 & 1000.0000 & 0.0 & 18 \\
\hline 34 & AKD828 & 5.0000 & 1.5000 & 0.7000 & 0.7000 & 500.0000 & 0.0 & $\mathbf{N}$ \\
\hline 35 & AKD908 & 5.0000 & 3.0000 & 2.0000 & 0.5000 & 1000.0000 & 0.0 & N \\
\hline 36 & AKD809 & 5.0000 & 3.0000 & 1.5000 & 0.5000 & 700.0000 & 0.0 & $\mathbf{N}$ \\
\hline 37 & AKD823 & 10.0000 & 2.0000 & 1.5000 & 0.5000 & 1000.0000 & 0.0 & L \\
\hline 38 & AKDB25 & 10.0000 & 2.0000 & 1.5000 & 1.0000 & 700.0000 & 0.0 & $\mathbf{N}$ \\
\hline 39 & AKD826 & 5.0000 & 2.0000 & 1.5000 & 0.5000 & 700.0000 & 0.0 & iv \\
\hline 40 & AKD8 27 & 7.0000 & 2.0000 & 1.0000 & 0.5000 & 1000.0000 & 0.0 & 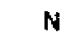 \\
\hline 4 & AKD810 & 3.0000 & 2.0000 & 1.5000 & 0.5000 & 1000.0000 & 0.0 & $\mathbf{N}$ \\
\hline 42 & AKOB11 & 3.0000 & 1.5000 & 1.5000 & 0.3000 & 1000.0000 & 0.0 & 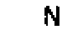 \\
\hline 43 & AKDB12 & 3.0000 & 1.5000 & 1.5000 & 0.3000 & 500.0000 & 0.0 & $\mathbf{N}$ \\
\hline 44 & AKD821 & 10.0000 & 2.0000 & 2.0000 & 0.7000 & 1000.0000 & 0.0 & $\mathbf{N}$ \\
\hline 85 & AKD820 & 10.0000 & 2.0000 & 1.0000 & 0.7000 & 1000.0000 & 0.0 & $\mathbf{N}$ \\
\hline$\not 4$ & AKD822 & 10.0000 & 2.0000 & 1.5000 & 0.7000 & 1500.0000 & 0.0 & $\mathbf{N}$ \\
\hline 47 & AKO816 & 3.0000 & 1.0000 & 1.0000 & 0.3000 & 300.0000 & 0.0 & $\mathbf{N}$ \\
\hline $4 r$ & AKD817 & 3.0000 & 1.0000 & 1.0000 & 0.3000 & 300.0000 & 0.0 & $\mathbf{N}$ \\
\hline 49 & AKD 18 & 5.0000 & 1.5000 & 0.7000 & 0.3000 & 300.0000 & 0.0 & \\
\hline 50 & AKDB 19 & 7.0000 & 2.0000 & 0.7000 & 0.5000 & 700.0000 & 0.0 & \\
\hline
\end{tabular}

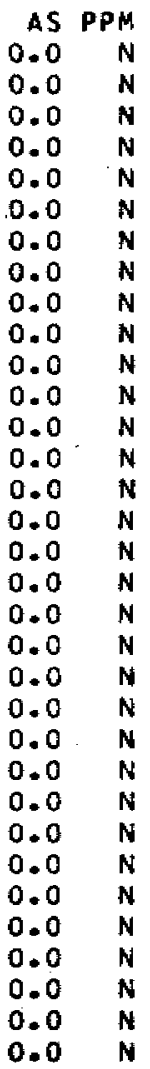

$\begin{array}{ll}\text { AU } & \text { PPM } \\ 0.0 & N \\ 0.0 & N \\ 0.0 & N \\ 0.0 & N \\ 0.0 & N \\ 0.0 & N \\ 0.0 & N \\ 0.0 & N \\ 0.0 & N \\ 0.0 & N \\ 0.0 & N \\ 0.0 & N \\ 0.0 & N \\ 0.0 & N \\ 0.0 & N \\ 0.0 & N \\ 0.0 & N \\ 0.0 & N \\ 0.0 & N \\ 0.0 & N \\ 0.0 & N \\ 0.0 & N \\ 0.0 & N \\ 0.0 & N \\ 0.0 & N \\ 0.0 & N \\ 0.0 & N \\ 0.0 & N \\ 0.0 & N \\ 0.0 & N \\ 0.0 & N \\ 0.0 & N \\ 0.0 & N \\ & N \\ \end{array}$

B PPH

$0.0 \quad L$

10.0000

0.0 L

0.0

0.0

0.0

10.0000

10.0000

$0.0 \mathrm{~N}$

10.0000

15.0000

15.0000
10.0000

10.0000
15.0000

10.0000

15.0000

10.0000

15.0000

10.0000

20.0000

0.0

10.0000

10.0000

10.0000

10.0000

15.0000

10.0000

10.0000

0.0 L

0.0

0.0
10.0000
BA PPM 300.0000 300.0000 1000.0000 1000.0000 1500.0000 700.0000 700.0000 1500.0000 300.0000 700.0000 500.0000 700.0000 300.0000 300.0000 300.0000 300.0000 700.0000 150.0000 300.0000 700.0000 700.0000 300.0000 300.0000 300.0000 150.0000 300.0000 300.0000 300.0000 300.0000 300.0000 150.0000 300.0000 300.0000 
SUMDUM C-4 STREAM SEDIMENTS

\begin{tabular}{llllr} 
SAMPLE & \multicolumn{1}{c}{ BE } & PPM & BI & PPM \\
AKD829 & 0.0 & $N$ & 0.0 & $N$ \\
AKD797 & 0.0 & $\mathrm{~L}$ & 0.0 & $\mathrm{~N}$ \\
AKD799 & 0.0 & $\mathrm{~L}$ & 0.0 & $\mathrm{~N}$ \\
AKD798 & 0.0 & $\mathrm{~L}$ & 0.0 & $\mathrm{~N}$ \\
AKD830 & 0.0 & $\mathrm{~L}$ & 0.0 & $\mathrm{~N}$ \\
AKD831 & 0.0 & $\mathrm{~L}$ & 0.0 & $\mathrm{~N}$ \\
AKD832 & 0.0 & $\mathrm{~L}$ & 0.0 & $\mathrm{~N}$ \\
AKD800 & 0.0 & $\mathrm{~L}$ & 0.0 & $\mathrm{~N}$ \\
AKD801 & 0.0 & $\mathrm{~N}$ & 0.0 & $\mathrm{~N}$ \\
AKD802 & 0.0 & $\mathrm{~N}$ & 0.0 & $\mathrm{~N}$ \\
AKD803 & 0.0 & $\mathrm{~N}$ & 0.0 & $\mathrm{~N}$ \\
AKD804 & 0.0 & $\mathrm{~N}$ & 0.0 & $\mathrm{~N}$ \\
AKD805 & 0.0 & $\mathrm{~L}$ & 0.0 & $\mathrm{~N}$ \\
AKD806 & 0.0 & $\mathrm{~L}$ & 0.0 & $\mathrm{~N}$ \\
AKD807 & 0.0 & $\mathrm{~L}$ & 0.0 & $\mathrm{~N}$ \\
AKD824 & 1.0000 & 0.0 & $\mathrm{~N}$ \\
AKD828 & 0.0 & $\mathrm{~L}$ & 0.0 & $\mathrm{~N}$ \\
AKD808 & 0.0 & $\mathrm{~N}$ & 0.0 & $\mathrm{~N}$ \\
AKD809 & 0.0 & $\mathrm{~L}$ & 0.0 & $\mathrm{~N}$ \\
AKD823 & 0.0 & $\mathrm{~N}$ & 0.0 & $\mathrm{~N}$ \\
AKD825 & 0.0 & $\mathrm{~L}$ & 0.0 & $\mathrm{~N}$ \\
AKD826 & 0.0 & $\mathrm{~L}$ & 0.0 & $\mathrm{~N}$ \\
AKD827 & 0.0 & $\mathrm{~L}$ & 0.0 & $\mathrm{~N}$ \\
AKDB10 & 0.0 & $\mathrm{~L}$ & 0.0 & $\mathrm{~N}$ \\
AKD811 & 0.0 & $\mathrm{~N}$ & 0.0 & $\mathrm{~N}$ \\
AKD812 & 0.0 & $\mathrm{~N}$ & 0.0 & $\mathrm{~N}$ \\
AKD821 & 0.0 & $\mathrm{~L}$ & 0.0 & $\mathrm{~N}$ \\
AKD820 & 0.0 & $\mathrm{~N}$ & 0.0 & $\mathrm{~N}$ \\
AKD822 & 0.0 & $\mathrm{~N}$ & 0.0 & $\mathrm{~N}$ \\
AKD 16 & 0.0 & $\mathrm{~L}$ & 0.0 & $\mathrm{~N}$ \\
AKD817 & 0.0 & $\mathrm{~N}$ & 0.0 & $\mathrm{~N}$ \\
AKD818 & 0.0 & $\mathrm{~L}$ & 0.0 & $\mathrm{~N}$ \\
AKD819 & 0.0 & $\mathrm{~L}$ & 0.0 & $\mathrm{~N}$
\end{tabular}

$\begin{array}{lr}\text { CD } & P P M \\ 0.0 & N \\ 0.0 & N \\ 0.0 & N \\ 0.0 & N \\ 0.0 & N \\ 0.0 & N \\ 0.0 & N \\ 0.0 & N \\ 0.0 & N \\ 0.0 & N \\ 0.0 & N \\ 0.0 & N \\ 0.0 & N \\ 0.0 & N \\ 0.0 & N \\ 0.0 & N \\ 0.0 & N \\ 0.0 & N \\ 0.0 & N \\ 0.0 & N \\ 0.0 & N \\ 0.0 & N \\ 0.0 & N \\ 0.0 & N \\ 0.0 & N \\ 0.0 & N \\ 0.0 & N \\ 0.0 & N \\ 0.0 & N \\ 0.0 & N \\ 0.0 & N \\ 0.0 & N \\ 0.0 & N \\ & \end{array}$

COPPM
30.0000
10.0000
20.0000
15.0000
20.0000
10.0000
7.0000
30.0000
20.0000
20.0000
10.0000
10.0000
15.0000
15.0000
30.0000
30.0000
20.0000
30.0000
30.0000
20.0000
20.0000
30.0000
20.0000
30.0000
20.0000
20.0000
30.0000
30.0000
20.0000
15.0000
15.0000
20.0000
30.0000

.

CR PPH
70.0000
70.0000
150.0000
150.0000
100.0000
70.0000
10.0000
150.0000
150.0000
70.0000
70.0000
30.0000
50.0000
50.0000
300.0000
150.0000
100.0000
150.0000
150.0000
30.0000
70.0000
70.0000
20.0000
150.0000
100.0000
150.0000
30.0000
30.0000
100.0000
30.0000
50.0000
50.0000
30.0000

CU PPM
7.0000
5.0000
30.0000
10.0000
10.0000
0.0
30.0000
50.0000
7.0000
10.0000
5.0000
5.0000
20.0000
15.0000
70.0000
30.0000
30.0000
20.0000
30.0000
50.0000
50.0000
20.0000
30.0000
10.0000
30.0000
30.0000
30.0000
100.0000
30.0000
30.0000
20.0000
30.0000
30.0000

3

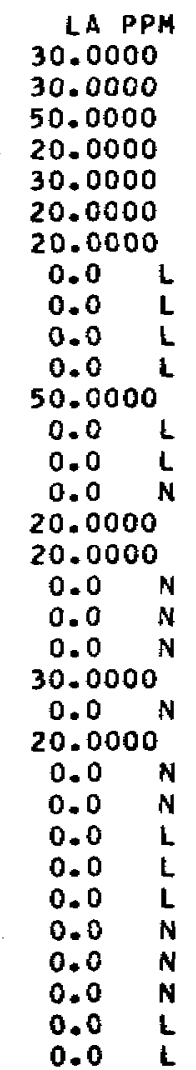

$\begin{array}{ll}M 0 & P P M \\ 0.0 & L \\ 0.0 & N \\ 0.0 & L \\ 0.0 & L \\ 0.0 & L \\ 0.0 & N \\ 0.0 & L \\ 0.0 & L \\ 0.0 & L \\ 0.0 & L \\ 0.0 & N \\ 0.0 & L \\ 0.0 & N \\ 0.0 & N \\ 0.0 & L \\ 0.0 & L \\ 0.0 & L \\ 0.0 & L \\ 0.0 & L \\ 0.0 & L \\ 0.0 & L \\ 0.0 & L \\ 0.0 & L \\ 0.0 & L \\ 0.0 & L \\ 0.0 & L \\ 0.0 & L \\ 0.0 & L \\ 0.0 & L \\ 0.0 & L \\ 0.0 & L \\ 0.0 & L \\ 0.0 & L\end{array}$

NB PPA 10.0000 0.0
10.0000

0.0 10.0000 $0.0 \mathrm{~L}$ 10.0000 10.0000 10.0000
0.0 0.0
10.0000 10.0000 10.0000 10.0000

10.0000

10.0000

10.0000 10.0000 0.0
10.0000 10.0000
10.0000 10.0000 10.0000

10.0000

10.0000

0.0

10.0000
0.0

0.0
10.0000

10.0000

10.0000

10.0000
NI PPH

15.0000

15.0000

70.0000

50.0000

30.0000

15.0000

$0.0 \mathrm{~L}$
50.0000

70.0000

15.0000

15.0000

20.0000

20.0000

15.0000

150.0000

70.0000

70.0000

70.0000

70.0000

20.0000

50.0000

30.0000

15.0000

50.0000

50.0000

30.0000

20.0000

20.0000

30.0000

20.0000

20.0000

20.0000

20.0000 
SUMOUM C-4 STREAM SEDIMENTS

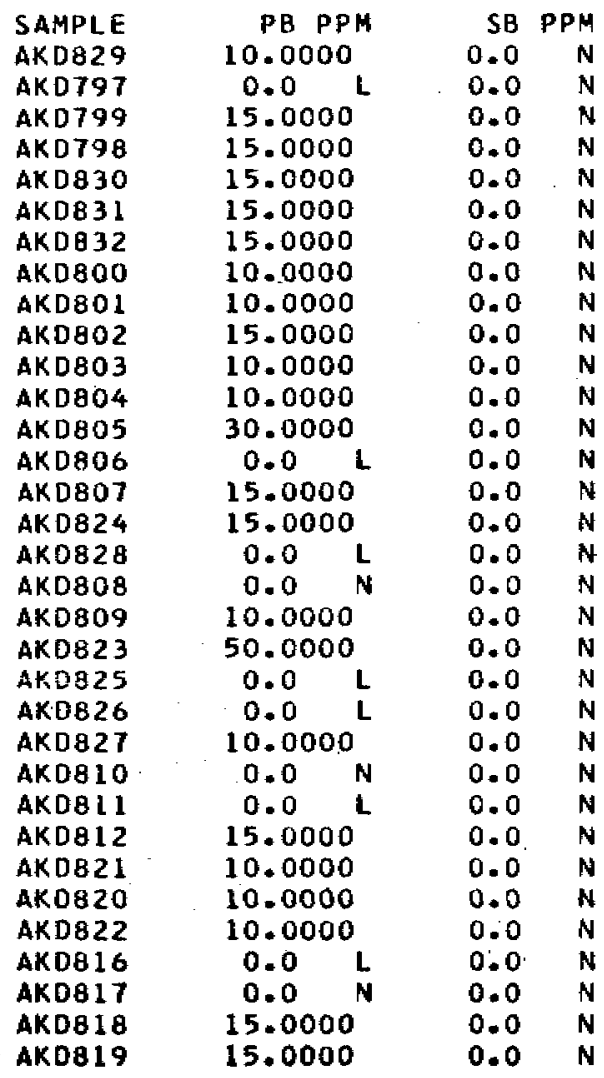

SC PPM 20.0000

15.0000

20.0000

15.0000

15.0000

15.0000

20.0000

20.0000

15.0000

15.0000

15.0000

15.0000

15.0000

30.0000

20.0000

15.0000

30.0000

20.0000

30.0000

20.0000

20.0000

20.0000

20.0000

20.0000

20.0000

30.0000

30.0000

30.0000

15.0000

15.0000

15.0000

20.0000

$\begin{array}{llllll}\text { SN PPM } & \text { SR PPM } & \text { V PPM } & \text { W PPM } \\ 0.0 & N & 300.0000 & 300.0000 & 0.0 & N \\ 0.0 & N & 300.0000 & 150.0000 & 0.0 & N \\ 0.0 & N & 300.0000 & 100.0000 & 0.0 & N \\ 0.0 & N & 300.0000 & 150.0000 & 0.0 & N \\ 0.0 & N & 200.0000 & 200.0000 & 0.0 & N \\ 0.0 & N & 300.0000 & 150.0000 & 0.0 & N \\ 0.0 & N & 300.0000 & 100.0000 & 0.0 & N \\ 0.0 & N & 150.0000 & 150.0000 & 0.0 & N \\ 0.0 & N & 200.0000 & 100.0000 & 0.0 & N \\ 0.0 & N & 200.0000 & 100.0000 & 0.0 & N \\ 0.0 & N & 200.0000 & 100.0000 & 0.0 & N \\ 0.0 & N & 200.0000 & 150.0000 & 0.0 & N \\ 0.0 & N & 300.0000 & 70.0000 & 0.0 & N \\ 0.0 & N & 300.0000 & 70.0000 & 0.0 & N \\ 0.0 & N & 200.0000 & 150.0000 & 0.0 & N \\ 0.0 & N & 300.0000 & 300.0000 & 0.0 & N \\ 0.0 & N & 150.0000 & 200.0000 & 0.0 & N \\ 0.0 & N & 200.0000 & 150.0000 & 0.0 & N \\ 0.0 & N & 150.0000 & 150.0000 & 0.0 & N \\ 0.0 & N & 150.0000 & 300.0000 & 0.0 & N \\ 0.0 & N & 200.0000 & 300.0000 & 0.0 & N \\ 0.0 & N & 150.0000 & 300.0000 & 0.0 & N \\ 0.0 & N & 150.0000 & 200.0000 & 0.0 & N \\ 0.0 & N & 150.0000 & 150.0000 & 0.0 & N \\ 0.0 & N & 150.0000 & 150.0000 & 0.0 & N \\ 0.0 & N & 150.0000 & 100.0000 & 0.0 & N \\ 0.0 & N & 200.0000 & 300.0000 & 0.0 & N \\ 0.0 & N & 200.0000 & 200.0000 & 0.0 & N \\ 0.0 & N & 150.0000 & 300.0000 & 0.0 & N \\ 0.0 & N & 150.0000 & 150.0000 & 0.0 & N \\ 0.0 & N & 100.0000 & 100.0000 & 0.0 & N \\ 0.0 & N & 150.0000 & 150.0000 & 0.0 & N \\ 0.0 & N & 150.0000 & 150.0000 & 0.0 & N\end{array}$

$Y P P$

20.0000

15.0000

20.0000

20.0000

15.0000

20.0000

20.0000

30.0000

20.0000

15.0000

10.0000

15.0000

15.0000

15.0000

15.0000

20.0000

15.0000

20.0000

15.0000

20.0000

15.0000

15.0000

30.0000

15.0000

15.0000

15.0000

30.0000

30.0000

30.0000

10.0000

10.0000
15.0000

20.0000

$\begin{array}{ll}\text { ZN } & \text { PPM } \\ 0.0 & N \\ 0.0 & N \\ 0.0 & \mathrm{~L} \\ 0.0 & N \\ 0.0 & N \\ 0.0 & N \\ 0.0 & N \\ 0.0 & \mathrm{~L} \\ 0.0 & \mathrm{~N} \\ 0.0 & \mathrm{~L} \\ 0.0 & \mathrm{~N} \\ 0.0 & \mathrm{~N} \\ 0.0 & \mathrm{~L} \\ 0.0 & \mathrm{~N} \\ 0.0 & \mathrm{~N} \\ 0.0 & \mathrm{~L} \\ 0.0 & \mathrm{~L} \\ 0.0 & \mathrm{~N} \\ 0.0 & \mathrm{~N} \\ 300.0000 \\ 0.0 & \mathrm{~L} \\ 0.0 & \mathrm{~N} \\ 0.0 & \mathrm{~N} \\ 0.0 & \mathrm{~L} \\ 0.0 & \mathrm{~N} \\ 0.0 & \mathrm{~N} \\ 0.0 & \mathrm{~L} \\ 0.0 & \mathrm{~N} \\ 0.0 & \mathrm{~L} \\ 0.0 & \mathrm{~N} \\ 0.0 & \mathrm{~N} \\ 0.0 & \mathrm{~N} \\ 0.0 & \mathrm{~N}\end{array}$

$2 \mathrm{PPM}$

500.0000

300.0000

300.0000

70.0000

00.0000

50.0000

150.0000

100.0000

70.0000

50.0000

300.0000

70.0000

20.0000

70.0000

70.0000

70.0000

70.0000

70.0000

70.0000

70.0000

70.0000

70.0000

70.0000

70.0000

70.0000

100.0000

1.00 .0000
70.0000

70.0000

70.0000

70.0000

50.0000

50.0000

70.0000 
SUMDUM C-4 STREAM SEDIMENTS

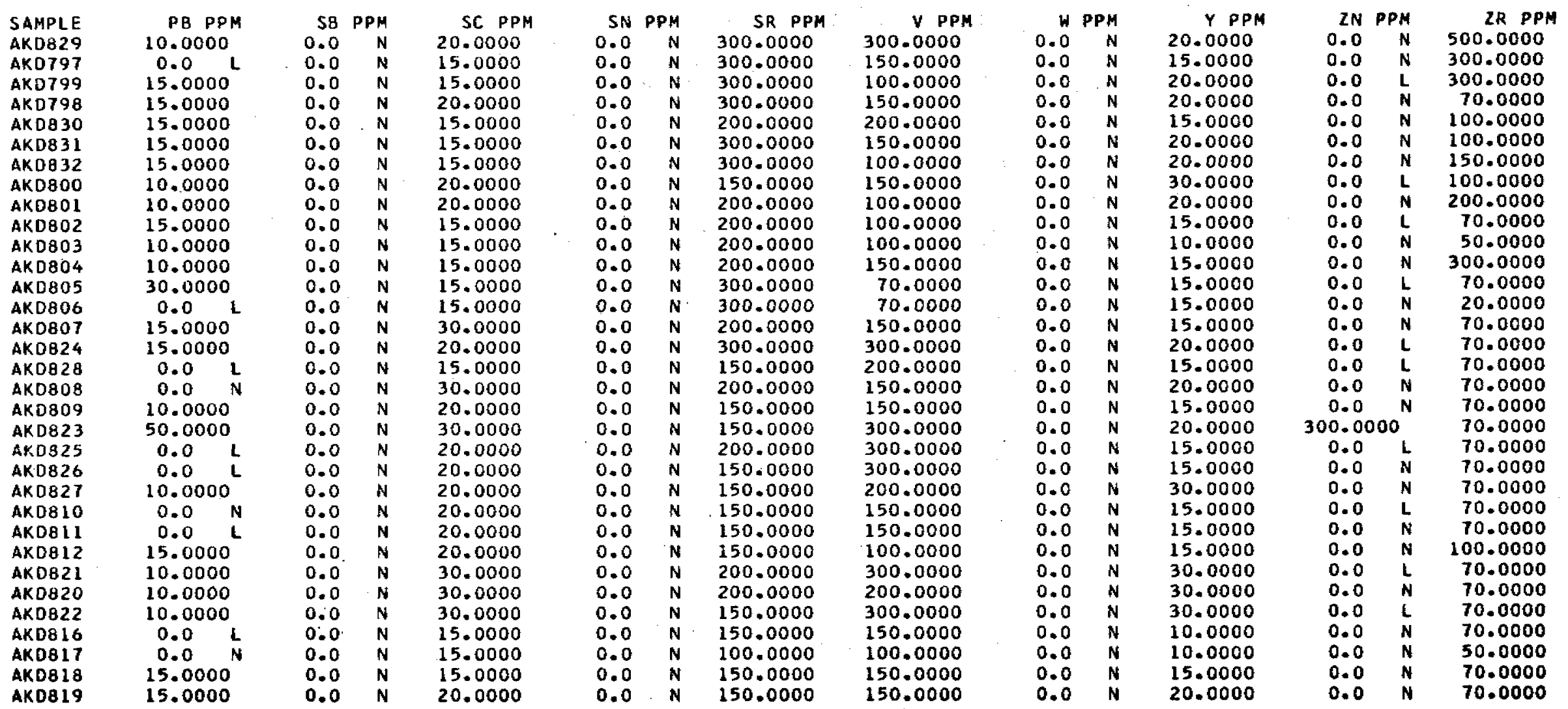


SUMDUM C-4 STREAH SEDIMENTS

\begin{tabular}{|c|c|c|c|c|}
\hline SAMPLE & PB PPM & SB & PPM & SC PPM \\
\hline AKD829 & 10.0000 & 0.0 & $N$ & 20.0000 \\
\hline AKDT97 & 0.0 & 0.0 & $N$ & 15.0000 \\
\hline AKD799 & 15.0000 & 0.0 & $\mathbf{N}$ & 15.0000 \\
\hline AKD798 & 15.0000 & 0.0 & $\mathbf{N}$ & 20.0000 \\
\hline AKD 830 & 15.0000 & 0.0 & N & 15.0000 \\
\hline AKO8 31 & 15.0000 & 0.0 & $\mathbf{N}$ & 15.0000 \\
\hline AKD832 & 15.0000 & 0.0 & $\mathbf{N}$ & 15.0000 \\
\hline AKD 800 & 10.0000 & 0.0 & N & 20.0000 \\
\hline AKD801 & 10.0000 & 0.0 & $\mathbf{N}$ & 20.0000 \\
\hline AKD 802 & 15.0000 & 0.0 & $\mathbf{N}$ & 15.0000 \\
\hline AKDBO3 & 10.0000 & 0.0 & $\mathrm{~N}$ & 15.0000 \\
\hline AKOOOO4 & 10.0000 & 0.0 & $N$ & 15.0000 \\
\hline AKDBO5 & 30.0000 & 0.0 & $\mathrm{~N}$ & 15.0000 \\
\hline AKD 806 & 0.0 & 0.0 & $\mathbf{N}$ & 15.0000 \\
\hline AKD 007 & 15.0000 & 0.0 & $\mathrm{~N}$ & 30.0000 \\
\hline AKDE24 & 15.0000 & 0.0 & $\mathrm{~N}$ & 20.0000 \\
\hline$A K D B 28$ & 0.0 & 0.0 & $N$ & 15.0000 \\
\hline AKDBO8 & 0.0 & 0.0 & $\mathbf{N}$ & 30.0000 \\
\hline AKD809 & 10.0000 & 0.0 & $\mathrm{~N}$ & 20.0000 \\
\hline AKD 823 & 50.0000 & 0.0 & $\mathbf{N}$ & 30.0000 \\
\hline AKD325 & 0.0 & 0.0 & $\mathrm{~N}$ & 20.0000 \\
\hline AK:0826 & 0.0 & 0.0 & $N$ & 20.0000 \\
\hline$A K D 827$ & 10.0000 & 0.0 & $\mathbf{N}$ & 20.0000 \\
\hline AKO810 & 0.0 & 0.0 & $\mathrm{~N}$ & 20.0000 \\
\hline AKO811 & 0.0 & 0.0 & $\mathbf{N}$ & 20.0000 \\
\hline AKD812 & 15.0000 & 0.0 & $\mathbf{N}$ & 20.0000 \\
\hline$A K D 821$ & 10.0000 & 0.0 & N & 30.0000 \\
\hline$\triangle K D \theta 20$ & 10.0000 & 0.0 & $N$ & 30.0000 \\
\hline AKD 822 & 10.0000 & 0.0 & $\mathrm{~N}$ & 30.0000 \\
\hline AKDB16 & 0.0 & 0.0 & $\mathbf{N}$ & 15.0000 \\
\hline AKD817 & 0.0 & 0.0 & $N$ & 15.0000 \\
\hline AKOB18 & 15.0000 & 0.0 & $\mathbf{N}$ & 15.0000 \\
\hline AKD819 & 15.0000 & 0.0 & $\mathbf{N}$ & 20.0000 \\
\hline
\end{tabular}

\begin{tabular}{|c|c|c|c|c|c|}
\hline SN & PPM & SR PPM & У РPM & 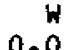 & PPM \\
\hline 0.0 & $N$ & 300.0000 & $\begin{array}{l}300.0000 \\
150.0000\end{array}$ & $\begin{array}{l}0.0 \\
0.0\end{array}$ & $\mathbf{N}$ \\
\hline 0.0 & $N$ & 300.0000 & 100.0000 & 0.0 & N \\
\hline 0.0 & $\mathbf{N}$ & 300.0000 & 150.0000 & 0.0 & $\mathbf{N}$ \\
\hline 0.0 & $\mathbf{N}$ & 200.0000 & 200.0000 & 0.0 & $\mathbf{N}$ \\
\hline 0.0 & $\mathbf{N}$ & 300.0000 & 150.0000 & 0.0 & $\mathbf{N}$ \\
\hline 0.0 & $\mathbf{N}$ & 300.0000 & 100.0000 & 0.0 & $N$ \\
\hline 0.0 & $\mathbf{N}$ & 150.0000 & 150.0000 & 0.0 & $\mathbf{N}$ \\
\hline 0.0 & N & 200.0000 & 100.0000 & 0.0 & $\mathbf{N}$ \\
\hline 0.0 & $\mathbf{N}$ & 200.0000 & 100.0000 & 0.0 & $\mathbf{N}$ \\
\hline 0.0 & N & 200.0000 & 100.0000 & 0.0 & $\mathbf{N}$ \\
\hline 0.0 & $\mathbf{N}$ & 200.0000 & 150.0000 & 0.0 & $\mathbf{N}$ \\
\hline 0.0 & $\mathbf{N}$ & 300.0000 & 70.0000 & 0.0 & $\mathbf{N}$ \\
\hline 0.0 & N & 300.0000 & 70.0000 & 0.0 & $\mathbf{N}$ \\
\hline 0.0 & $N$ & 200.0000 & 150.0000 & 0.0 & $\mathbf{N}$ \\
\hline 0.0 & $\mathbf{N}$ & 300.0000 & 300.0000 & 0.0 & $\mathbf{N}$ \\
\hline 0.0 & $\mathbf{N}$ & 150.0000 & 200.0000 & 0.0 & $\mathbf{N}$ \\
\hline 0.0 & $\mathbf{N}$ & 200.0000 & 150.0000 & 0.0 & $\mathbf{N}$ \\
\hline 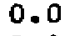 & N & 150.0000 & 150.0000 & 0.0 & $\mathbf{N}$ \\
\hline 0.0 & N & 150.0000 & 300.0000 & 0.0 & $\mathbf{N}$ \\
\hline 0.0 & $\mathbf{N}$ & 200.0000 & 300.0000 & 0.0 & $\mathbf{N}$ \\
\hline 0.0 & $\mathbf{N}$ & 150.0000 & 300.0000 & 0.0 & $\mathbf{N}$ \\
\hline & $\mathbf{N}$ & 150.0000 & 200.0000 & 0.0 & $\mathbf{N}$ \\
\hline 0.0 & $\mathrm{~N}$ & 150.0000 & 150.0000 & 0.0 & $\mathbf{N}$ \\
\hline 0 . & $\mathbf{N}$ & 150.0000 & 150.0000 & 0.0 & $\mathbf{N}$ \\
\hline 0.0 & $\mathbf{N}$ & 150.0000 & 100.0000 & 0.0 & $\mathbf{N}$ \\
\hline 0.0 & N & 200.0000 & 300.0000 & 0.0 & $\mathbf{N}$ \\
\hline 0.0 & $\mathbf{N}$ & 200.0000 & 200.0000 & 0.0 & $\mathbf{N}$ \\
\hline 0 . & $\mathbf{N}$ & 150.0000 & 300.0000 & 0.0 & $\mathbf{N}$ \\
\hline 0 & $\mathbf{N}$ & 150.0000 & 150.0000 & 0.0 & $N$ \\
\hline 0.0 & $N$ & 100.0000 & 100.0000 & 0.0 & $\mathbf{N}$ \\
\hline 0.0 & $\mathbf{N}$ & 150.0000 & 150.0000 & 0.0 & $\mathbf{N}$ \\
\hline & $\mathbf{N}$ & 0000 & 150.0000 & 0.0 & $\mathbf{N}$ \\
\hline
\end{tabular}

\begin{tabular}{ccc} 
YPPM & \multicolumn{2}{c}{ ZN PPM } \\
20.0000 & 0.0 & $N$ \\
15.0000 & 0.0 & $N$ \\
20.0000 & 0.0 & $L$ \\
20.0000 & 0.0 & $N$ \\
15.0000 & 0.0 & $N$ \\
20.0000 & 0.0 & $N$ \\
20.0000 & 0.0 & $N$ \\
30.0000 & 0.0 & $L$ \\
20.0000 & 0.0 & $N$ \\
15.0000 & 0.0 & $L$ \\
10.0000 & 0.0 & $N$ \\
15.0000 & 0.0 & $N$ \\
15.0000 & 0.0 & $L$ \\
15.0000 & 0.0 & $N$ \\
15.0000 & 0.0 & $N$ \\
20.0000 & 0.0 & $L$ \\
15.0000 & 0.0 & $L$ \\
20.0000 & 0.0 & $N$ \\
15.0000 & 0.0 & $N$ \\
20.0000 & 300.0000 \\
15.0000 & 0.0 & $L$ \\
15.0000 & 0.0 & $N$ \\
30.0000 & 0.0 & $N$ \\
15.0000 & 0.0 & $L$ \\
15.0000 & 0.0 & $N$ \\
15.0000 & 0.0 & $N$ \\
30.0000 & 0.0 & $L$ \\
30.0000 & 0.0 & $N$ \\
30.0000 & 0.0 & $L$ \\
10.0000 & 0.0 & $N$ \\
10.0000 & 0.0 & $N$ \\
15.0000 & 0.0 & $N$ \\
20.0000 & 0.0 & $N$ \\
& &
\end{tabular}

2R PPA

500.0000

300.0000

300.0000

70.0000

100.0000

100.0000

150.0000

150.0000

200.0000

70.0000

50.0000

300.0000

70.0000

20.0000

70.0000

70.00

70.0000

70.0000

70.0000

70.0000

70.0000

70.0000

70.0000

70.0000

70.0000

70.0000

70.0000
100.0000

70.0000

70.0000

70.0000

70.0000

50.000

50.0000

70.0000 


$\begin{array}{ll}\text { SAMPLE } & \text { AU PPM } \\ \text { AKDB29 } & 0.0200 \mathrm{~L} \\ \text { AKD797 } & 0.0200 \mathrm{~L} \\ \text { AKD799 } & 0.0200 \mathrm{~L} \\ \text { AKD798 } & 0.0200 \mathrm{~L} \\ \text { AKDB30 } & 0.0200 \mathrm{~L} \\ \text { AKDB31 } & 0.0200 \mathrm{~L} \\ \text { AKD832 } & 0.0200 \mathrm{~L} \\ \text { AKDB00 } & 0.0200 \mathrm{~L} \\ \text { AKD801 } & 0.0200 \mathrm{~L} \\ \text { AKDB02 } & 0.0200 \mathrm{~L} \\ \text { AKDB03 } & 0.0200 \mathrm{~L} \\ \text { AKD804 } & 0.0200 \mathrm{~L} \\ \text { AKD805 } & 0.0200 \mathrm{~L} \\ \text { AKD806 } & 0.0200 \mathrm{~L} \\ \text { AKD807 } & 0.0200 \mathrm{~L} \\ \text { AKDB24 } & 0.0200 \mathrm{~L} \\ \text { AKDB28 } & 0.0200 \mathrm{~L} \\ \text { AKD808 } & 0.0200 \mathrm{~L} \\ \text { AKD809 } & 0.0200 \mathrm{~L} \\ \text { AKD823 } & 0.0200 \mathrm{~L} \\ \text { AKDB25 } & 2.2000 \\ \text { AKD826 } & 0.0200 \mathrm{~L} \\ \text { AKDB27 } & 0.0200 \mathrm{~L} \\ \text { AKDB10 } & 0.0200 \mathrm{~L} \\ \text { AKDB11 } & 0.0200 \mathrm{~L} \\ \text { AKDB12 } & 0.0200 \mathrm{~L} \\ \text { AKDB21 } & 0.0200 \mathrm{~L} \\ \text { AKD820 } & 0.0200 \mathrm{~L} \\ \text { AKD822 } & 0.0200 \mathrm{~L} \\ \text { AKDB16 } & 0.0200 \mathrm{~L} \\ \text { AKD817 } & 0.0200 \mathrm{~L} \\ \text { AKD818 } & 0.0200 \mathrm{~L} \\ \text { AKDB19 } & 0.0200 \mathrm{~L}\end{array}$


A470 GRAPHICAL ANALYSIS

TI TLE

SUMDUM $\mathrm{C}-4$ STREAM SEDIMENTS

COLUMN IDENTIFIERS FE PCT MG PC BE PPM BI PPM PB PPM SB PPM AU PPM

CA PCT $C D P P M$ SC PPM $\begin{array}{ll}\text { TI } & \text { PCT } \\ \text { CO } & \text { PPM }\end{array}$ SN PPM

MN PPM

CR PPM

SR PPM

AG PPM CU PPM COPM
PPM

AS PPM LA PPM W PPM

AU PPM

MO PPM

$Y$ PPM
B PPM
NB PPM
BA PPM
NI PPM

ZR PPM 
FREQUENCY TABLE FOR COLUMN

1 I FE PCT

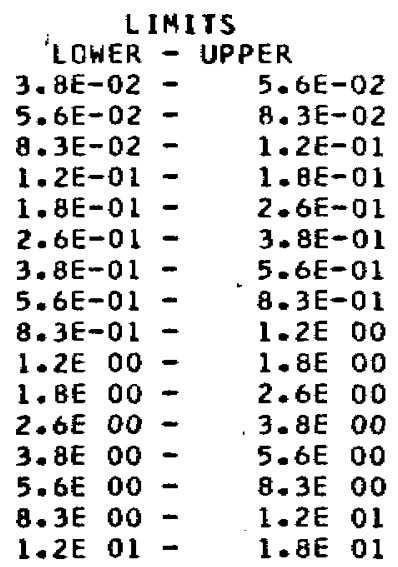

$\begin{array}{cccc}\text { FREQ } & \text { FREQ } & \text { PERCENT } & \text { PERCENT } \\ & \text { CUM } & \text { FREQ } & \text { FREQ CUM } \\ 0 & 0 & 0.0 & 0.0 \\ 0 & 0 & 0.0 & 0.0 \\ 0 & 0 & 0.0 & 0.0 \\ 0 & 0 & 0.0 & 0.0 \\ 0 & 0 & 0.0 & 0.0 \\ 0 & 0 & 0.0 & 0.0 \\ 0 & 0 & 0.0 & 0.0 \\ 0 & 0 & 0.0 & 0.0 \\ 0 & 0 & 0.0 & 0.0 \\ 0 & 0 & 0.0 & 0.0 \\ 0 & 0 & 0.0 & 0.0 \\ 13 & 13 & 39.39 & 39.39 \\ 11 & 24 & 33.33 & 72.73 \\ 2 & 26 & 6.06 & 78.79 \\ 6 & 32 & 18.18 & 96.97 \\ 1 & 33 & 3.03 & 100.00\end{array}$

HISTOGRAM FOR COLUMN 1 ( FE PCT)

3.0E 00 $\quad x \times x \times x \times x \times x \times x \times x \times x \times x \times x \times x \times x \times x \times x \times x \times x \times x \times x \times x \times x$

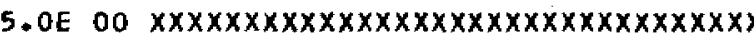

7. OE OO $\times \times \times \times \times X$

1.0E $01 \quad \times \times \times \times \times \times \times \times \times \times \times \times \times \times \times \times \times x$

en $1.5 E$ o1 $x \times x$

$\begin{array}{ccccccc}N & \text { L } & \text { H } & \text { B } & T & \text { G } & \text { ANALYTICAL VALUES } \\ 0 & 0 & 0 & 0 & 0 & 0 & 33 \\ 0.0 & 0.0 & & & 0.0 & 0.0 & \end{array}$

MAXIMUM $=1.50000 E 01$

MINIMUM $=3.00000 E 00$

GEOMETRIC MEAN $=4.89349 E$ OO

GEOMETRIC DEVIATION $=1.63286 E 00$ 
FREQUENCY TABLE FOR COLUMN 21 MG PCT)

\begin{tabular}{|c|c|c|c|c|c|c|}
\hline \multicolumn{3}{|c|}{ LIMII } & FREQ & $\begin{array}{r}\text { FREQ } \\
\text { CUM }\end{array}$ & $\begin{array}{c}\text { PERCENT } \\
\text { FREQ }\end{array}$ & $\begin{array}{l}\text { PERCENT } \\
\text { FREQ CUM }\end{array}$ \\
\hline $.8 E-02$ & - & $2.6 \mathrm{E}-02$ & 0 & 0 & 0.0 & 0.0 \\
\hline$E-02$ & - & 3. $8 E-02$ & 0 & 0 & 0.0 & 0.0 \\
\hline$E-02$ & - & $5.6 \mathrm{E}-02$ & 0 & 0 & 0.0 & 0.0 \\
\hline .02 & - & -02 & 0 & 0 & 0. & 0.0 \\
\hline-02 & - & $1.2 E-01$ & 0 & 0 & 0. & 0.0 \\
\hline 01 & - & $1.8 \mathrm{E}-01$ & 0 & 0 & 0.0 & 0.0 \\
\hline$E-01$ & - & $2.6 E-01$ & 0 & 0 & 0.0 & 0.0 \\
\hline$E-01$ & - & $3.8 E-01$ & 0 & G & 0.0 & 0.0 \\
\hline$E-01$ & - & $5.6 E-01$ & 0 & c & 0. & 0.0 \\
\hline 01 & 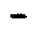 & B. $3 E-01$ & 0 & $c$ & 0 . & 0.0 \\
\hline $3 E-01$ & - & $1.2 \mathrm{E} \quad 00$ & 2 & 2 & 6.06 & 6.0 \\
\hline ZE 00 & 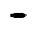 & $1.8 \mathrm{E} \quad 00$ & 12 & 14 & 36.36 & 42.4 \\
\hline $\begin{array}{l}\text { - } 8 E 00 \\
\text { GE } 00\end{array}$ & - & $\begin{array}{ll}2.6 \mathrm{E} & 00 \\
3.8 \mathrm{E} & 00\end{array}$ & $\begin{array}{r}14 \\
5\end{array}$ & $\begin{array}{l}28 \\
33\end{array}$ & $\begin{array}{l}42.42 \\
15.15\end{array}$ & $\begin{array}{l}85 \\
00\end{array}$ \\
\hline
\end{tabular}

HISTOGRAM FOR CDLUMN 2 ( MG PCT)

1. OE $00 \quad \times \times \times \times \times \times$

1. 5E O0 $x \times x \times x \times x \times x \times x \times x \times x \times x \times x \times x \times x \times x \times x \times x \times x \times x \times x \times x$

2.0E 00 $x \times x \times x \times x \times x \times x \times x \times x \times x \times x \times x \times x \times x \times x \times x \times x \times x \times x \times x \times x \times x \times x$

3.0E $00 \quad x \times \times \times \times \times x \times x \times x \times x \times x$

en

$\begin{array}{cc}N & L \\ 0 & 0 \\ 0.0 & 0.0\end{array}$

$H$
0

B
$\mathbf{0}$

7
0
0.0

ANALYTICAL

VALUES

MAXIMUM $=3.00000 E 00$

MINIMUM $=1.00000 E$ O0

GEDMETRIC MEAN $=1.83668 E$ OO

GEOMETRIC DEVIATION $=1.3251$ AE OO 
FREQUENCY TABLE FOR - COLUMN

3 (CA PCT ,

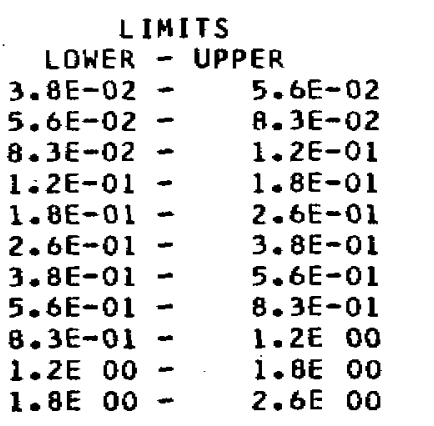

FREQ
0
0
0
0
0
0
0
3
5
17
8

FREG
CUM
0
0
0
0
0
0
0
3
8
25
33

$\begin{array}{cc}\text { PERCENT } & \text { PERCENT } \\ \text { FREQ } & \text { FREQ CUM } \\ 0.0 & 0.0 \\ 0.0 & 0.0 \\ 0.0 & 0.0 \\ 0.0 & 0.0 \\ 0.0 & 0.0 \\ 0.0 & 0.0 \\ 0.0 & 0.0 \\ 9.09 & 9.09 \\ 15.15 & 24.24 \\ 51.52 & 75.76 \\ 24.24 & 100.00\end{array}$

HISTOGRAM FDR COLUMN

3 ( CA PCT,

\section{OE-01 $\times \times \times \times \times \times \times \times x$}

1.0E $00 \quad x \times x \times x \times x \times x \times x \times x \times x$

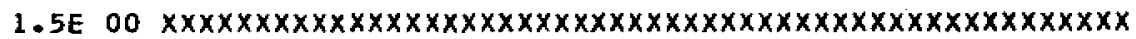

2. OE OO $\times x \times x \times x \times x \times x \times x \times x \times x \times x \times x \times x \times x$

\begin{tabular}{ccccccc}
$N$ & $L$ & $H$ & & & \multicolumn{2}{c}{ ANALYTICAL } \\
0 & 0 & 0 & 0 & $T$ & $G$ & VALUES \\
0.0 & 0.0 & & 0 & 0.0 & 0.0 & 33
\end{tabular}

of MAXIMUM $=2.00000 \mathrm{E} 00$

MINIMUM $=7.00000 E-01$

GEOMETRIC MEAN $=1.41126 E$ OO

GEOMETRIC DEVIATION $=1.36540 E$ OO 
FREQUENCY TABLE FOR COLUMN

4 ( TI PCT)

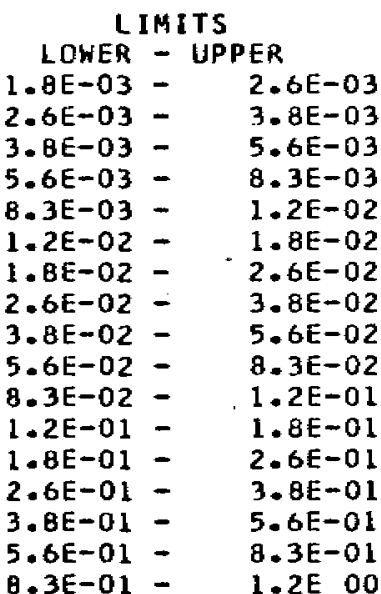

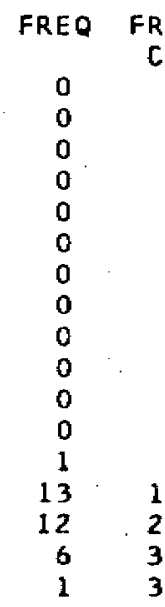

$\begin{array}{cc}\text { PERCENT } & \text { PERCENT } \\ \text { FREQ } & \text { FREQ CUM } \\ 0.0 & 0.0 \\ 0.0 & 0.0 \\ 0.0 & 0.0 \\ 0.0 & 0.0 \\ 0.0 & 0.0 \\ 0.0 & 0.0 \\ 0.0 & 0.0 \\ 0.0 & 0.0 \\ 0.0 & 0.0 \\ 0.0 & 0.0 \\ 0.0 & 0.0 \\ 0.0 & 0.0 \\ 3.03 & 3.03 \\ 39.39 & 42.42 \\ 36.36 & 78.79 \\ 18.18 & 96.97 \\ 3.03 & 100.00\end{array}$

HISTOGRAM FOR COLUMN

4 ( TI PCT ।

\subsection{E-01 $X X X$}

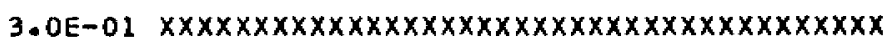

5. OE-01 $x \times x \times x \times x x \times x \times x \times x \times x \times x \times x \times x \times x \times x \times x \times x \times x \times x \times x$

7. OE $-01 \quad x \times x \times x \times x \times x \times x \times x \times x \times x \times$

1.0E OO XXX

$\begin{array}{ccccccc}N & L & H & & & & \text { ANALYTICAL } \\ 0 & 0 & 0 & 0 & 0 & 0 & \text { VALUES } \\ 0.0 & 0.0 & & & 0.0 & 0.0 & 33\end{array}$

MAXIMUM $=1.00000 E 00$

MINIMUM $=2.00000 E-01$

GEOMETRIC MEAN $=4.31725 E-01$

GEOMETRIC DEVIATIDN $=1.46565 E 00$ 
FREQUENCY TABLE FOR COLUMN 5 ( MN PPM)

\begin{tabular}{|c|c|c|c|c|c|c|c|c|}
\hline \multicolumn{5}{|c|}{$\begin{array}{c}\text { LINITS } \\
\text { LOWER - UPPER }\end{array}$} & FREQ & $\begin{array}{l}\text { FREQ } \\
\text { CUN }\end{array}$ & $\begin{array}{c}\text { PERCENT } \\
\text { FREQ }\end{array}$ & $\begin{array}{l}\text { PERCENT } \\
\text { FREO CUM }\end{array}$ \\
\hline $3 \mathrm{E}$ & 00 & - & $1.2 E$ & 01 & 0 & 0 & 0.0 & 0.0 \\
\hline $.2 \mathrm{E}$ & 01 & - & $1.8 E$ & 01 & 0 & 0 & 0.0 & 0.0 \\
\hline $.8 \mathrm{E}$ & 01 & - & $2.6 E$ & 01 & 0 & 0 & 0.0 & 0.0 \\
\hline - & 01 & - & $3.8 E$ & 01 & 0 & 0 & 0.0 & 0.0 \\
\hline & 01 & - & $5.6 \mathrm{E}$ & 01 & 0 & 0 & 0.0 & 0.0 \\
\hline $.6 E$ & 01 & - & $8.3 E$ & 01 & 0 & 0 & 0.0 & 0.0 \\
\hline $3 \mathrm{E}$ & 01 & - & L. $2 E$ & 02 & 0 & 0 & 0.0 & 0.0 \\
\hline $.2 \mathrm{E}$ & 02 & - & $1.8 E$ & 02 & 0 & 0 & 0.0 & 0.0 \\
\hline $1.8 \mathrm{E}$ & 02 & - & $2.6 \mathrm{E}$ & 02 & 0 & 0 & 0.0 & 0.0 \\
\hline & 02 & - & $3 \cdot \mathrm{BE}$ & 02 & 5 & 5 & 15.15 & 15.15 \\
\hline $.8 E$ & 02 & - & $5.6 \mathrm{E}$ & 02 & 4 & 9 & $12 \cdot 12$ & 27.27 \\
\hline $.6 \mathrm{E}$ & 02 & - & $8.3 E$ & 02 & 12 & 21 & 36.36 & 63.64 \\
\hline$\cdot 3 \mathrm{E}$ & 02 & - & $1.2 \mathrm{E}$ & 03 & 11 & 32 & $\begin{array}{r}33.33 \\
3.03\end{array}$ & 96.97 \\
\hline & & & & & & 33 & 3.03 & 100.00 \\
\hline
\end{tabular}

HISTOGRAM FOR COLUMN 5 I MN PPM )

3.0E $02 \quad x \times x \times x \times x \times x \times x \times x \times x$

5. OE $02 \quad x \times x \times x \times x \times x \times x \times$

7. OE $02 \quad x \times x \times x \times x \times x \times x \times x \times x \times x \times x \times x \times x \times x \times x \dot{x} \times x \times x \times x \times x \times x$

1. OE $03 \quad x \times x \times x \times x \times x \times x \times x \times x \times x \times x \times x \times x \times x \times x \times x \times x \times x$

1.5E $03 \times \times X$

我

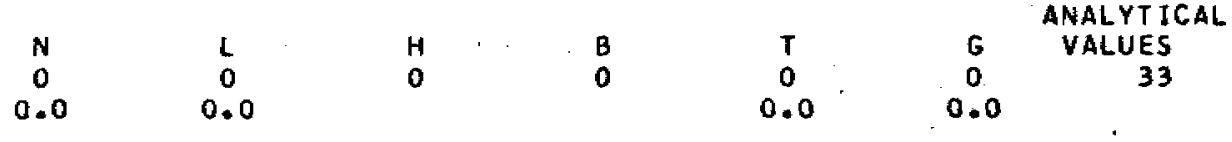

MAXIMUM $=1.50000 E 03$

MINIMUM $=3.00000 E 02$

GEOMETRIC MEAN $=6.81225 E 02$

GEDMETRIC DEVIATION = $1.54192 E$ DO 
FREQUENCY TABLE FOR COLUMN

6 (AG PPM )

LIMITS
LOWER - UPPER

FREQ FREQ PERCENT
CUM FREQ

PERCENT

FREO FREQ CUM

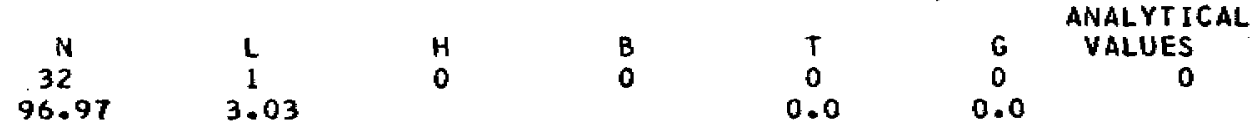

MAXIMUM $=-9.99900 E 48$

MINIMUM $=9.99900 E 48$

GEOMETRIC MEAN $=9.99900 E 48$

GEOMETRIC DEVIATION $=9.99900 E 48$ 
FREQUENCY TABLE FOR COLUMN 7 I AS PPM ।

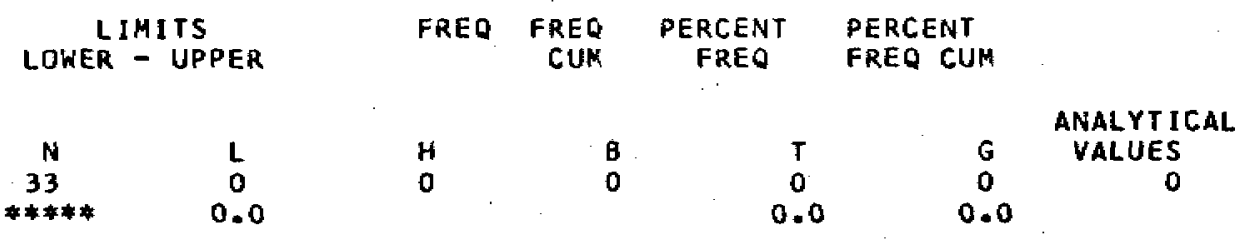

MAXIMUM $=-9.99900 E 4 \mathrm{~B}$

MINIMUN $=9.99900 E 48$

GEOMETRIC MEAN $=9.99900 E 48$

GEOMETRIC DEVIATION = $9.99900 E 48$ 


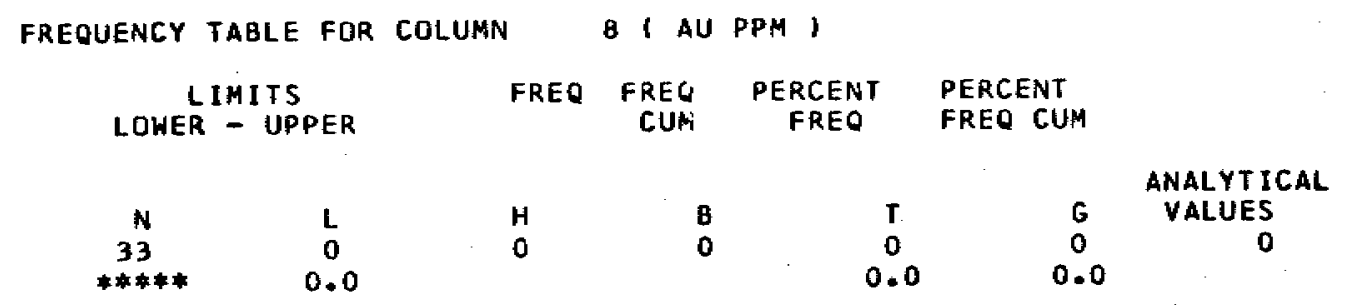

MAXIMUM $=-9.99900 E 48$

MINIMUM $=9.99900 E 48$.

GEOMETRIC MEAN $=9.99900 E 48$

GEOMETRIC DEVIATION $=9.99900 E 48$ 
FREQUENCY TABLE FOR COLUMN

9 ( B PPM)

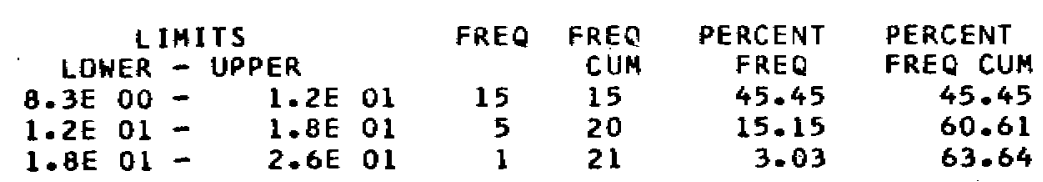

HISTOGRAM FOR COLUMN 91 B PPM )

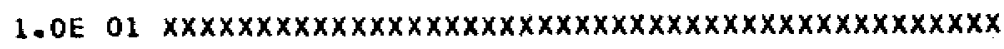

1.5E $01 \quad \times \times \times \times \times \times \times \times \times \times \times x \times x \times$

2. OE $01 \times \times X$

$\begin{array}{ccccccc}N & L & H & B & T & G & \text { ANALYTICAL } \\ 4 & B & 0 & 0 & 0 & 0 & 21 \\ 12.12 & 24.24 & & & 0.0 & 0.0 & \end{array}$

MAXIMUM $=2.00000 E$ OI

MINIMUM $=1.00000 E 01$

GEOMETRIC MEAN $=1.13829 E$ OI

D 
FREQUENCY TABLE FOR COLUMN

10 ( BA PPM)

\begin{tabular}{|c|c|c|c|c|}
\hline & & & & \\
\hline 1.5 & AI & & PER & \\
\hline $6 \mathrm{E}$ & 01 & - & 3. $8 \mathrm{E}$ & 01 \\
\hline$B E$ & 01 & - & $5.6 \mathrm{E}$ & 01 \\
\hline $6 \mathrm{E}$ & 01 & & $8.3 E$ & 01 \\
\hline $3 t$ & 01 & - & $1.2 \mathrm{E}$ & 02 \\
\hline & 02 & & & 02 \\
\hline $\mathbf{E}$ & 02 & & $2.6 \mathrm{E}$ & 02 \\
\hline $6 \mathrm{E}$ & 02 & - & $3.8 \mathrm{E}$ & 02 \\
\hline & 02 & & $6 E$ & 02 \\
\hline & 02 & & $3 \mathrm{E}$ & 02 \\
\hline & $\begin{array}{l}02 \\
03\end{array}$ & - & $\begin{array}{l}1.2 \mathrm{E} \\
1.8 \mathrm{E}\end{array}$ & $\begin{array}{l}03 \\
03\end{array}$ \\
\hline
\end{tabular}

$\begin{array}{cc}\text { FREQ } & \text { FREQ } \\ & \text { CUM } \\ 0 & 0 \\ 0 & 0 \\ 0 & 0 \\ 0 & 0 \\ 0 & 0 \\ 3 & 3 \\ 0 & 3 \\ 18 & 21 \\ 1 & 22 \\ 7 & 29 \\ 2 & 31 \\ 2 & 33\end{array}$

PERCENT

FREO FREQ CUM

$0.0 \quad 0.0$

$0.0 \quad 0.0$

$0.0-0.0$

$0.0 \quad 0.0$

$\begin{array}{ll}0.0 & 0.0 \\ 9.09 & 9.09\end{array}$

$0.0 \quad 9.09$

$54.55 \quad 63.64$

$3.03 \quad 66.67$

$21.21 \quad 87.88$

$6.06-93.94$

$6.06 \quad 100.00$

HISTOGRAM FOR COLUMN 10 ( BA PPM )

1.5E $02 \times \times \times \times \times \times \times \times \times$

$2.0 E \quad 02$

3.0E 02 $x \times x \times x \times x \times x \times x \times x \times x \times x \times x \times x \times x \times x \times x \times x \times x \times x \times x \times x \times x \times x \times x \times x \times x \times x \times x \times x \times x$

5. OE $02 \times x \times$

ఱ

7. OE $02 \quad x \times x \times x \times x \times x \times x \times x \times x \times x \times x \times x$.

1.0E $03 \times \times \times \times \times x$

1.5E $03 \times \times \times \times \times \times x$

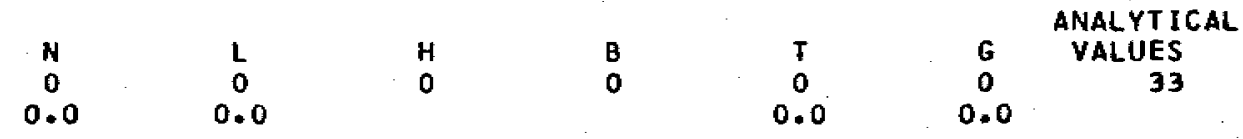

MAXIMUM $=1.50000 E 03$

MINIMUM $=1.50000 E 02$

GEOMETRIC MEAN $=4.06048 E$ O2

GEOMETRIC DEVIATION $=1.83484$ E OO 
FREQUENCY TABLE FOR COLUMN

11 BE PPM ।

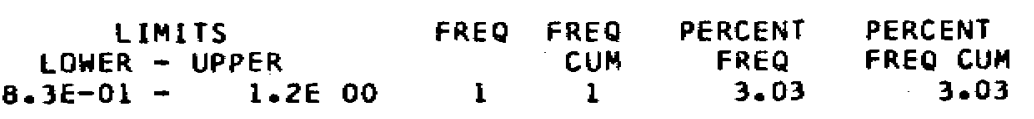

histogram for COLUMn 11 | Be PPM ।

1. OE $00 \times \times \times$

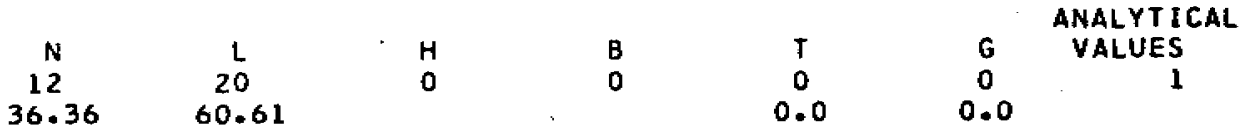

MAXIMUM $=1.00000 E 00$

MINIMUM $=1.00000 E 00$

GEOMETRIC MEAN $=1.00000 E$ DO

GEOMETRIC DEVIATION $=9.99900$ E 48 
FREQUENCY TABLE FOR COLUMN

12 BI PPM ।

$$
\text { LIMITS }
$$

FREQ FREQ

PERCENT

FREQ

PERCENT

FREQ CUM

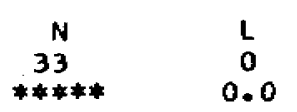

$H$
0

B
0

$T$
0
0.0

ANALYTICAL

VALUES

MAXIMUM $=-9.99900 \mathrm{E} 48$

MINIMUM $=9.99900 E 48$

GEOMETRIC MEAN $=9.99900 E 48$

GEOMETRIC DEVIATION $=9.99900$ E 48 
FREQUENCY TABLE FOR COLUMN

13 ( CO PPM ।

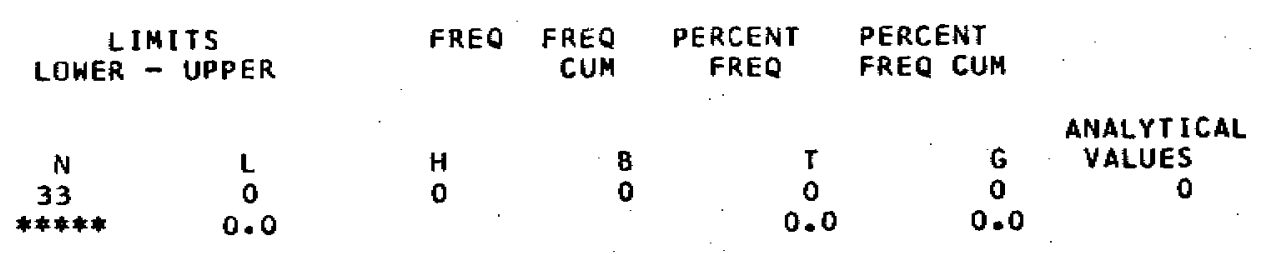

MAXIMUM $=-9.99900 E \quad 48$

MINIMUM $=9.99900 E 48$

GEOMETRIC MEAN $=9.99900 E 48$

GEOHETRIC DEVIATIDN $=9.99900 E 48$ 
FREQUENCY TABLE FOR COLUMN

14 \& CO PPM I

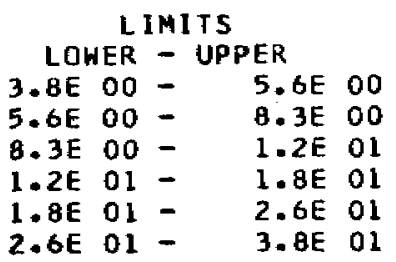

$\begin{array}{cccc}\text { FREQ } & \text { FREQ } & \text { PERCENT } & \text { PERCENT } \\ & \text { CUM } & \text { FREQ } & \text { FREQ CUM } \\ 0 & 0 & 0.0 & 0.0 \\ 1 & 1 & 3.03 & 3.03 \\ 4 & 5 & 12.12 & 15.15 \\ 5 & 10 & 15.15 & 30.30 \\ 12 & 22 & 36.36 & 66.67 \\ 11 & 33 & 33.33 & 100.00\end{array}$

HISTOGRAM FOR COLUMN

14 (CO PPM)

7. OE OO $\times X X$

1. OE $01 \times \times \times \times \times x \times x \times x \times x$

1.5E $01 \quad x \times \times \times \times \times \times \times \times \times \times \times \times \times x$

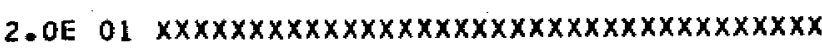

3. OE $01 \quad x \times x \times x \times x \times x \times x \times x \times x \times x \times x \times x \times x \times x \times x \times x \times x \times x$

$\theta$

$\begin{array}{cc}N & 1 \\ 0 & 0 \\ 0.0 & 0.0\end{array}$

$H$
0

B
$\mathbf{0}$

$T$
0
0.0

ANALYTICAL

VALUES

MAXIMUM $=3.00000 E$ O1

MINIMUM $=7.00000 E 00$

GEOMETRIC MEAN $=1.95203 \mathrm{E} 01$

GEOMETRIC DEVIATION $=1.49809$ E 00 
FREQUENCY TABLE FOR COLUMN

15 ( CR PPM)

\begin{tabular}{|c|c|c|c|c|}
\hline & & $I M I$ & & \\
\hline & NER & & & \\
\hline 86 & 00 & - & $5.6 \mathrm{E}$ & 00 \\
\hline & 00 & 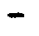 & B. $3 E$ & \\
\hline 36 & 00 & - & $1.2 \mathrm{E}$ & \\
\hline $2 \mathrm{E}$ & 01 & - & $E$ & \\
\hline & 01 & - & & \\
\hline $2.6 E$ & 01 & - & $3.8 \mathrm{E}$ & \\
\hline $8 E$ & 01 & 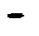 & $6 \mathrm{E}$ & \\
\hline $6 E$ & 01 & - & $3 E$ & \\
\hline $3 \mathrm{E}$ & 01 & - & & \\
\hline $2 \mathrm{E}$ & 02 & - & $8 \mathrm{E}$ & \\
\hline.$e$ & $\begin{array}{l}02 \\
02\end{array}$ & - & & \\
\hline
\end{tabular}

$\begin{array}{cc}\text { FREQ } & \text { FRE } \\ & \text { CUM } \\ 0 & 0 \\ 0 & 0 \\ 1 & 1 \\ 0 & 1 \\ 1 & 2 \\ 6 & 8 \\ 4 & 12 \\ 7 & 19 \\ 4 & 23 \\ 9 & 32 \\ 0 & 32 \\ 1 & 33\end{array}$

$\begin{array}{cc}\text { PERCENT } & \text { PERCENT } \\ \text { FREQ } & \text { FREQ CUM } \\ 0.0 & 0.0 \\ 0.0 & 0.0 \\ 3.03 & 3.03 \\ 0.0 & 3.03 \\ 3.03 & 6.06 \\ 18.18 & 24.24 \\ 12.12 & 36.36 \\ 21.21 & 57.58 \\ 12.12 & 69.70 \\ 27.27 & 96.97 \\ 0.0 & 96.97 \\ 3.03 & 100.00\end{array}$

HISTOGRAM FOR COLUMN

15 ( CR PPM )

1. OE OI $x \times x$

$1.5 \mathrm{E} \mathrm{Ol}$

2. 0 E $01 \times \times \times$

3. OE $01 \quad x \times x \times x \times x \times x \times x \times x \times x \times x x$

$\underset{\infty}{\infty}$

5. OE $01 \quad \times x \times x \times x \times x \times x \times x$

7.0E $01 \quad x \times \times \times \times \times \times \times \times \times \times \times \times \times \times \times \times \times \times \times x$

1. OE $02 \quad x \times x \times x \times x \times x \times x \times$

1.5E $02 \quad x \times x \times x \times x \times x \times x \times x \times x \times x \times x \times x \times x \times x \times x x$

$2.0 E 02$

3. OE $02 \times \times X$

\begin{tabular}{|c|c|c|c|c|c|c|}
\hline & & 4 & $\mathbf{A}$ & $T$ & G & ANALYT ICAL. \\
\hline 0 & 0 & 0 & 0 & 0 & 0 & 33 \\
\hline
\end{tabular}

MAXIMUM $=3.00000 E 02$

MINIMUM $=1.00000 E 01$

GEOMETRIC MEAN $=7.02389 E 01$

GEOMETRIC DÉVIATION $=2.11579 E 00$ 
FREQUENCY TABLE FOR COLUMN

16 I CU PPM I

\begin{tabular}{|c|c|c|c|c|}
\hline & & I M I & & \\
\hline & JER & & ER & \\
\hline & $\begin{array}{l}00 \\
00\end{array}$ & $\overline{-}$ & & $\begin{array}{l}00 \\
00\end{array}$ \\
\hline $3 E$ & 00 & - & $1.2 \mathrm{E}$ & 0 \\
\hline $2 E$ & 01 & - & $1.8 \mathrm{E}$ & \\
\hline & 01 & - & $6 E$ & \\
\hline & 01 & - & $8 E$ & \\
\hline & 01 & - & $6 \mathrm{E}$ & \\
\hline & $\begin{array}{l}01 \\
01\end{array}$ & - & $\begin{array}{l}8.3 \mathrm{E} \\
1.2 \mathrm{E}\end{array}$ & \\
\hline
\end{tabular}

FREO
3
2
4
1
4
13
3
1
1

$\begin{array}{crr}\text { FREQ } & \text { PERCENT } & \text { PERCENT } \\ \text { CUM } & \text { FREQ } & \text { FREQ CUM } \\ 3 & 9.09 & 9.09 \\ 5 & 6.06 & 15.15 \\ 9 & 12.12 & 27.27 \\ 10 & 3.03 & 30.30 \\ 14 & 12.12 & 42.42 \\ 27 & 39.39 & 81.82 \\ 30 & 9.09 & 90.91 \\ 31 & 3.03 & 93.94 \\ 32 & 3.03 & 96.97\end{array}$

HISTOGRAM FOR COLUMM 16 ( CU PPM)

5.0E $00 \quad \times \times \times \times \times \times \times \times x$

7. OE $00 \times \times \times \times \times \times$

1.0E $01 \times \times \times \times \times \times \times \times \times \times \times x$

1.5E OL $\times \times X$

2.0E $01 \times x \times \times \times \times \times x \times x \times x$

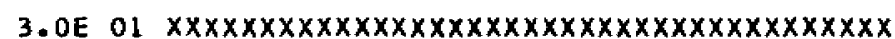

5.0E O1 $\times \times \times \times \times \times \times \times x$

7. OE $01 \quad x \times X$

1.0E O2 XXX

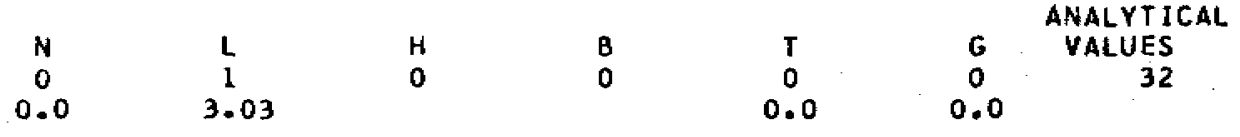

MAXIMUM $=1.00000 E 02$

MINIMUM $=5.00000 E 00$

GEOMETRIC MEAN $=2.1001 L$ OL

GEOMETRIC DEVIATION $=2.17492 \mathrm{E} 00$ 
FREQUENCY TABLE FDR COLUMN 17 \& LA PPM 1

\begin{tabular}{|c|c|c|c|c|c|c|}
\hline $\begin{array}{l}\text { LIH } \\
\text { LOWER } \\
1.8 E \text { OL } \\
2.6 \text { OL } \\
3.8 E \text { Ol }\end{array}$ & $\begin{array}{l}\text { MITS } \\
-\quad \text { UPPER } \\
-\quad 2.6 \mathrm{E} \\
-\quad 3.8 \mathrm{E} \\
-\quad 5.6 \mathrm{E}\end{array}$ & $\begin{array}{l}01 \\
01 \\
01\end{array}$ & FREO & $\begin{array}{c}\text { FREQ } \\
\text { CUM } \\
6 \\
10 \\
12\end{array}$ & $\begin{array}{c}\text { PERCENT } \\
\text { FREQ } \\
18.18 \\
12.12 \\
6.06\end{array}$ & $\begin{array}{l}\text { PERCENT } \\
\text { FREQ CUM } \\
18.18 \\
30.30 \\
36.36\end{array}$ \\
\hline
\end{tabular}

HISTOGRAM FOR COLUMN 17 LA PPM

2. OE O1 $\times \times \times \times \times \times \times \times \times \times \times \times \times \times \times \times \times \times x$

3. 0 E $01 \quad x \times x \times x \times x \times x \times x \times$

5. OE $01 \times \times \times \times \times \times$

$\begin{array}{cccccccc}N & L & H & B & T & G & \text { VALUES } \\ 10 & 11 & 0 & 0 & 0 & 0 & 12 \\ 30.30 & 33.33 & & & 0.0 & 0.0 & \end{array}$

MAXIMUM $=5.00000 E 01$

MINIMUM $=2.00000 E 01$

GEOMETRIC MEAN $=2.66716 E 01$

$\underset{0}{0}$

GEOMETRIC DEVIATION = 1.41811 E 00 
FREQUENCY TABLE FOR COLUMN

$18(M O P P M)$

\begin{tabular}{|c|c|c|c|c|c|c|}
\hline LOWE & $\begin{array}{l}\text { TS } \\
\text { UPPER }\end{array}$ & FREO & $\begin{array}{l}\text { FREQ } \\
\text { CUM }\end{array}$ & $\begin{array}{c}\text { PERCENT } \\
\text { FREQ }\end{array}$ & $\begin{array}{l}\text { PERCENT } \\
\text { FREQ CUM }\end{array}$ & \\
\hline $\begin{array}{c}\frac{N}{5} \\
15.15\end{array}$ & $\begin{array}{c}2 \\
28 \\
84.85\end{array}$ & $\begin{array}{l}H \\
0\end{array}$ & $\begin{array}{l}B \\
0\end{array}$ & $\begin{array}{c}T \\
0 \\
0.0\end{array}$ & $\begin{array}{c}G \\
0 \\
0.0\end{array}$ & $\begin{array}{c}\text { ANALYT ICAL } \\
\text { VALUES } \\
0\end{array}$ \\
\hline
\end{tabular}

MAXIMUM $=-9.99900 E 48$

MINIMUM $=9.99900 E 48$

GEOMETRIC MEAN $=9.99900 E 48$

GEOMETRIC DEVIATION $=9.99900$ E 48 
FREQUENCY TABLE FOR COLUMN 191 NB PPM ।

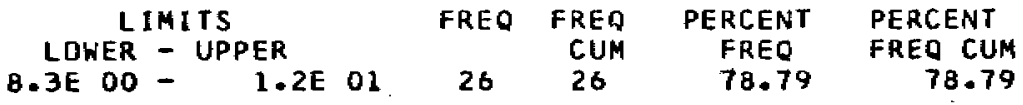

HISTOGRAM FOR COLUMN 19 ( NB PPM)

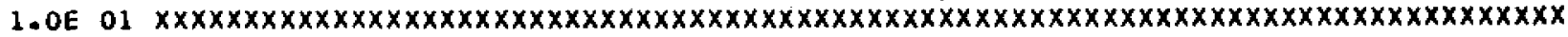

$\begin{array}{cc}N & L \\ 0 & 7 \\ 0.0 & 21.21\end{array}$

H

\section{B}

$\substack{1 \\ 0 \\ 0.0}_{0.0}$

ANALYTICAL

MAXIMUM $=1.00000 E 01$

MINIMUM $=1.00000 E 01$

GEOHETRIC MEAN $=9.99985 E$ OO

GEOMETRIC DEVIATION $=1.00566$ E 00 
FREQUENCY TABLE FOR COLUHN 20 ( NI PPM)

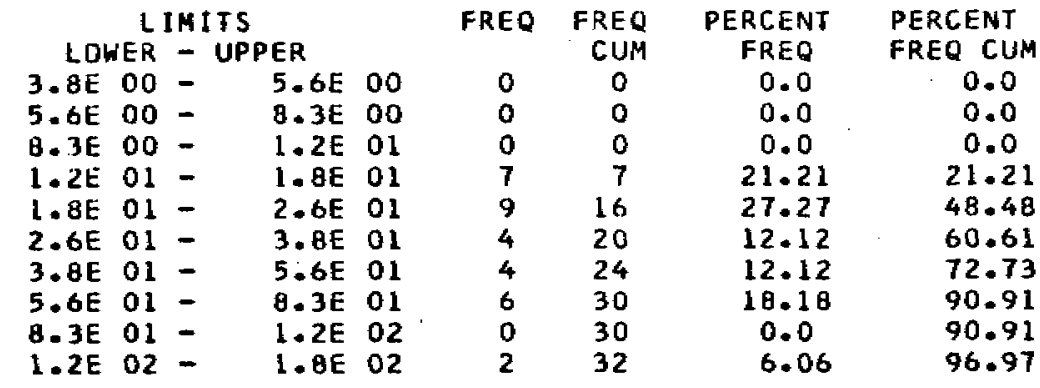

HISTOGRAM FOR COLUMN 201 NI PPM 1

1.5E $01 \quad x \times x \times x \times x \times x \times x \times x \times x \times x \times x \times x$

2.0E $01 \quad x \times x \times x \times x \times x \times x \times x \times x \times x \times x \times x \times x \times x \times x$

3.0E $01 \quad x \times \times \times \times \times \times \times \times \times \times \times$

5.0E $01 \quad x \times x \times x \times x \times x \times x x$

7. OE $01 \quad x \times x \times x \times x \times x \times x \times x \times x \times x \times$

$\underset{\omega}{3}$

I.OE 02

1.5E $02 \times \times \times \times \times X$

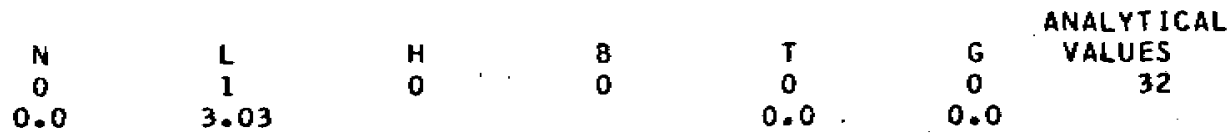

MAXIMUM $=1.50000 E 02$

MINIMUM $=1.50000 E$ O1

GEOMETRIC MEAN $=3.17799 E$ OL

GEOMETRIC DEVIATION $=2.01744$ E 00 


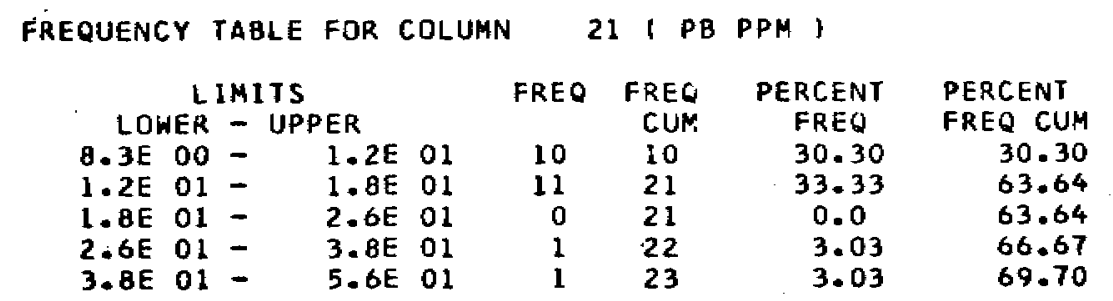

\section{HISTOGRAM FOR COLUMN 21 ( PB PPM)}

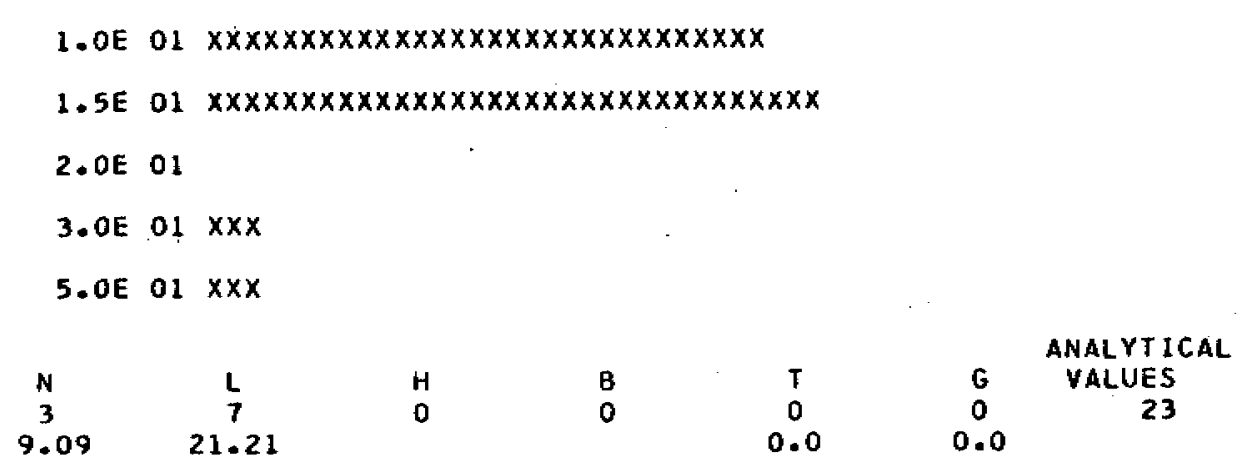

$\begin{array}{ccccccc}N & & & & & \text { ANALYTICAL } \\ 3 & L & H & B & T & G & \text { VALUES } \\ 9.09 & 21.21 & 0 & 0 & 0 & 0 & 23\end{array}$

$\checkmark$ MAXIMUM $=5.00000 E 01$

MINIMUM $=1.00000 E 01$
GEOMETRIC MEAN $=1.36567 \mathrm{E} 01$

GEOMETRIC DEVIATION $=1.479200^{\circ}$ OO 
FREQUENCY TABLE FOR COLUMN 22 ( SB PPM )

\begin{tabular}{|c|c|c|c|}
\hline $\begin{array}{l}\text { LIMITS } \\
\text { WER - UPPER }\end{array}$ & FREO & $\begin{array}{l}\text { FREC } \\
\text { CUM }\end{array}$ & $\begin{array}{c}\text { PERCENT } \\
\text { FREQ }\end{array}$ \\
\hline
\end{tabular}

$\begin{array}{ccccccc}N & L & H & B & & & \text { ANALYTICAL } \\ 33 & 0 & 0 & 0 & 0 & 0 & \text { VALUES } \\ * * * * * & 0.0 & & & 0.0 & 0.0 & 0\end{array}$

MAXIMUM $=-9.99900 E 48$

MINIMUM $=9.99900 E 48$

GEOMETRIC MEAN $=9.99900 E 48$

GEOMETRIC DEVIATION $=9.99900 E 48$ 
FREQUENCY TABLE FDR COLUMN 23 ( SC PPM I

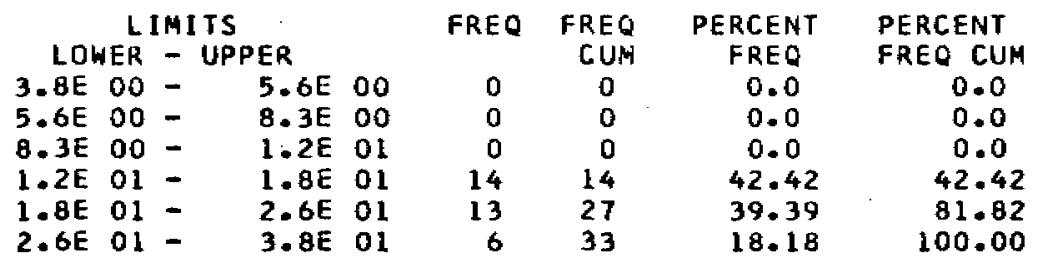

HISTOGRAM FOR COLUMN 23 ( SC PPM)

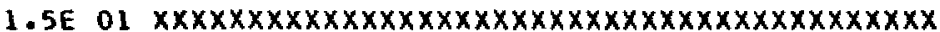

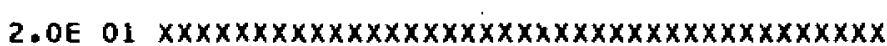

3. OE $01 \quad \times \times \times \times \times \times \times \times \times \times \times \times \times \times \times \times \times \times$

$\begin{array}{ccccccc}N & L & H & & & & \text { ANALYTICAL } \\ 0 & 0 & 0 & 0 & T & G & \text { VALUES } \\ 0.0 & 0.0 & & 0 & 0 & 0 & 33\end{array}$

MAXIMUM $=3.00000 E$ OI

MINIMUM $=1.50000 E$ OI

$\vec{\sigma}$ GEOMETRIC MEAN $=1.90563 \mathrm{E}$ OI

GEOMETRIC OEVIATION $=1.28952 E 00$ 
Frequency table fOR COLUMN 24 (- SN PPM I

LIMITS
LOWER - UPPER

FREQ FREQ PERCENT

PERCENT

CUN FREQ FREQ CUM

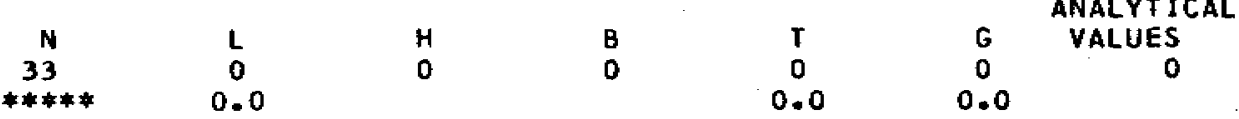

MAXIMUM $=-9.99900 E 48$

MINIMUM $=9.99900 E 48$

GEOMETRIC MEAN $=9.99900 E 48$

GEOMETRIC DEVIATION $=9.99900 E 48$ 
FREQUENCY TABLE FOR COLUMN

25 I SR PPM ।

\begin{tabular}{cccccr} 
LIMITS & FREQ & FREQ & PERCENT & \multicolumn{1}{c}{ PERCENT } \\
LOWER - UPPER & & & CUM & FREQ & FREQ CUM \\
$8.3 E 01-$ & $1.2 E$ O2 & 1 & 1 & 3.03 & 3.03 \\
$1.2 E$ O2 - & $1.8 E$ O2 & 13 & 14 & 39.39 & 42.42 \\
$1.8 E 02-$ & $2.6 E$ O2 & 10 & 24 & 30.30 & 72.73 \\
$2.6 E 02-$ & $3.8 E 02$ & 9 & 33 & 27.27 & 100.00
\end{tabular}

HISTOGRAM FOR COLUMN

25 ( SR PPM ।

$1.0 E 02 \times \times X$

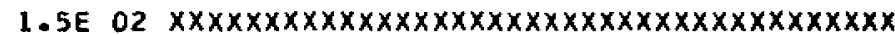

2.0E $02 \quad x \times x \times x \times x \times x \times x \times x \times x \times x \times x \times x \times x \times x \times x \times x x$

3.0E $02 \quad x \times x \times x \times x \times x \times x \times x \times x \times x \times x \times x \times x \times x \times x$

$\begin{array}{ccccccc}N & \text { L } & H & & & & \text { ANALYTICAL } \\ 0 & 0 & 0 & 0 & T & G & \text { VALUES } \\ 0.0 & 0.0 & & 0 & 0 & 0 & 33\end{array}$

MAXIMUM $=3.00000 E 02$

MINIMUM $=1.00000 \mathrm{E} 02$

$\infty$ GEDMETRIC MEAN $=1.95303 \mathrm{E} \cdot 02$

GEDMETRIC DEVIATION $=1.35966 E$ OO 
FREQUENCY TABLE FOR COLUMN

$261 \checkmark$ PPM

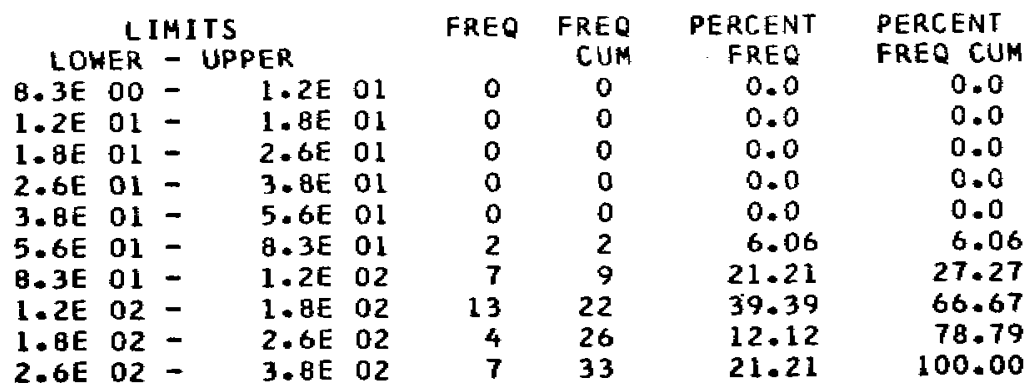

HISTOGRAM FOR COLUMN 26 I $Y$ PPH ।

7. OE $01 \times \times \times \times \times \times$

1. $0 \mathrm{E} \quad 02 \times \times \times \times \times \times \times \times \times \times \times \times \times \times \times \times \times \times \times \times x$

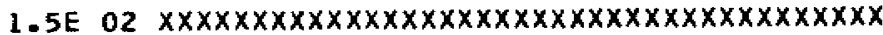

2.OE $02 \times x \times x \times x \times x \times x \times x$

3. OE $02 \quad x \times x \times x \times \times \times \times x \times \times \times \times \times \times \times \times \times \times x$

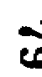

$\begin{array}{cc}\mathrm{N} & \mathrm{L} \\ 0 & 0 \\ 0.0 & 0.0\end{array}$

$B$
0

0.0

0.0

(n)

MAXIMUM $=3.00000 E 02$

MINIMUM $=7.00000 E 01$

GEOMETRIC MEAN $=1.57642 E 02$

GEOMETRIC DEVIATION $=1.53783$ E 00 
FREQUENCY TABLE FOR COLUMN 271 W PPM? LIMITS
LOWER - UPPER

FREQ FREQ PERCENT
CUM FREQ

PERCENT

FREQ CUM

$\begin{array}{cc}\text { N } & L \\ 33 & 0 \\ * * * * & 0.0\end{array}$

$H$
$\mathbf{O}$

B

$T$
0
0.0

ANALYTICAL

VALUES

MAXIMUM $=-9.99900 E 48$

MINIMUM $=9.99900 E 48$

GEOMETRIC MEAN $=9.99900 E 48$

GEOMETRIC DEVIATION $=9.99900 E 48$ 
FREQUENCY TABLE FOR COLUMN, $28, Y$ PPM ।

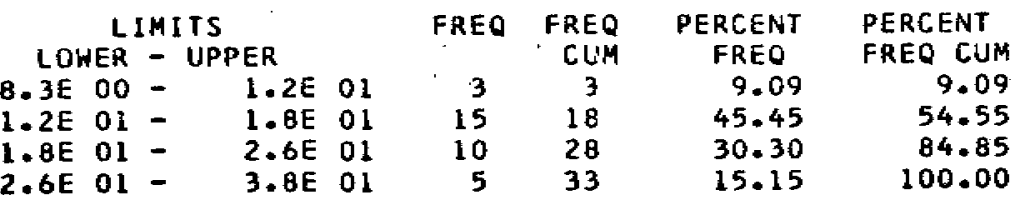

HISTOGRAM FOR COLUMN $28, Y$ PPM I

1.0E $01 \quad \times \times \times \times \times \times \times \times \times$

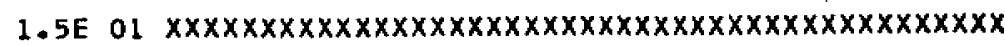

2. OE $01 \quad x \times x \times x \times x \times x \times x \times x \times \times \times \times \times \times \times x \times x \times x \times x \times x \times$

3.0E $01 \times x \times x \times x \times x \times x \times x \times x \times$

$\begin{array}{ccccccc}N & L & H & & & \text { ANALYTICAL } \\ 0 & L & H & B & T & G & 33 \\ 0.0 & 0.0 & 0 & 0 & 0 & 0 & 33 \text { VALUES }\end{array}$

$\infty$ MAXIMUM $=3.00000 E 01$

MINIMUM $=1.00000 E 01$

GEOMETRIC MEAN $=1.75206 E 01$

GEOMETRIC DEVIATION $=.1 .35318 E$ OO 
FREQUENCY TABLE FOR COLUMN 29 ( IN PPM)

$\begin{array}{cccccc}\text { LIMITS } & \text { FREO } & \text { FREQ } & \text { PERCENT } & \text { PERCENT } \\ \text { COWER } & \text { FUM } & \text { FREQ } & \text { FREQ CUM } \\ 1.8 E 02- & 2.6 E 02 & 0 & 0 & 0.0 & 0.0 \\ 2.6 E 02- & 3.8 E 02 & 1 & 1 & 3.03 & 3.03\end{array}$

HISTOGRAM FOR COLUMN 29 ( 2N PPM)

3. OE O2 XXX

\begin{tabular}{ccccccc}
$N$ & & & & & \multicolumn{2}{c}{ ANALYTICAL } \\
22 & 10 & $H$ & $B$ & $T$ & $G$ & VALUES \\
66.67 & 30.30 & 0 & 0 & 0 & 0 & 1
\end{tabular}

MAXIMUM $=3.00000 E 02$

MINIMUM $=3.00000 E$ O2

GEOMETRIC MEAN $=2.99999 E$ O2

GEOMETRIC DEVIATIDN $=9.99900 E 48$

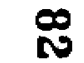


FREQUENCY TABLE FOR COLUMN $3012 R$ PPM 1

\begin{tabular}{|c|c|c|c|c|c|c|c|c|}
\hline \multicolumn{5}{|c|}{ LIMITS } & FREO & $\begin{array}{l}\text { FREQ } \\
\text { CUM }\end{array}$ & $\begin{array}{c}\text { PERCENT } \\
\text { FREO }\end{array}$ & $\begin{array}{l}\text { PERCENT } \\
\text { FREQ CUM }\end{array}$ \\
\hline $8.3 \mathrm{E}$ & 00 & - & $1.2 \mathrm{E}$ & 01 & 0 & 0 & 0.0 & 0.0 \\
\hline $1.2 \mathrm{E}$ & 01 & - & $1 . B E$ & 01 & 0 & 0 & 0.0 & 0.0 \\
\hline $1.8 \mathrm{E}$ & 01 & - & $2.6 \mathrm{E}$ & 01 & 1 & i & 3.03 & 3.03 \\
\hline $2.6 \mathrm{E}$ & 01 & - & $3.8 \mathrm{E}$ & 01 & 0 & 1 & 0.0 & 3.03 \\
\hline $3.8 \mathrm{E}$ & 01 & - & $5.6 E$ & 01 & 2 & 3 & 6.06 & 9.09 \\
\hline $5.6 E$ & 01 & - & B. $3 \mathrm{E}$ & 01 & 20 & 23 & 60.61 & 69.70 \\
\hline $3 E$ & 01 & - & $1.2 \mathrm{E}$ & 02 & 4 & 27 & 12.12 & 81.82 \\
\hline $2 \mathrm{E}$ & 02 & - & $1.8 E$ & 02 & 1 & 28 & 3.03 & .85 \\
\hline 1 & 02 & - & $2.6 E$ & 02 & 1 & 29 & 3.03 & 87.88 \\
\hline & 02 & - & $3 . \mathrm{BE}$ & 02 & 3 & 32 & 9.09 & 96.97 \\
\hline & 02 & - & $5.6 \mathrm{E}$ & 02 & 1 & 33 & 3.03 & .00 \\
\hline
\end{tabular}

HISTOGRAM FOR COLUMN $30(2 R$ PPH )

2.0E $01 \times \times x$

3. OE 01

5. OE $01 \times \times \times \times \times \times$

7.0E $01 \quad x \times x \times x \times x \times x \times x \times x \times x \times x \times x \times x \times x \times x \times x \times x \times x \times x \times x \times x \times x \times x \times x \times x \times x \times x \times x \times x \times x \times x \times x \times x \times x$

孞

1. 0 E $02 \quad x \times x \times x \times x \times x \times x \times$

1. $5 \mathrm{E} 02 \times \times \times$

2.0E $02 \times \times \times$

3. $0 E \quad 02 \quad x \times \times \times \times \times \times \times x$

5. OE $02 \times \times X$

$\begin{array}{ccccccc}N & \text { L } & \text { H } & \text { B } & \text { T } & \text { G } & \text { ANALYTICAL } \\ 0 & 0 & 0 & 0 & 0 & 0 & 33 \\ 0.0 & 0.0 & & & 0.0 & 0.0 & \end{array}$

MAXIMUM $=5.00000 E 02$

MINIMUM $=2.00000 E$ O1

GEDMETRIC MEAN $=8.82476 E$ OI

GEOMETRIC DEVIATION $=1.86530 E 00$ 
FREQUENCY TABLE FOR COLUMA

31 I AU PPM ।

\begin{tabular}{|c|c|c|c|c|c|c|}
\hline LOWER & IM & ER & FREO & $\begin{array}{r}\text { FREQ } \\
\text { CLUM }\end{array}$ & $\begin{array}{c}\text { PERCENT } \\
\text { FREQ }\end{array}$ & $\begin{array}{l}\text { PERCENT } \\
\text { FREQ CUM }\end{array}$ \\
\hline 1. $8 \mathrm{E}-02$ & - & $2.6 E-02$ & 0 & 0 & 0.0 & 0.0 \\
\hline $2.6 \mathrm{E}-02$ & - & 3. $\mathrm{aE}-02$ & 0 & 0 & 0.0 & 0.0 \\
\hline 3. $8 \mathrm{E}-02$ & - & $5.6 E-02$ & 0 & 0 & 0.0 & 0.0 \\
\hline $5.6 E-02$ & - & $8.3 E-02$ & 0 & 0 & 0.0 & 0.0 \\
\hline $8.3 E-02$ & - & $1.2 \mathrm{E}-01$ & 0 & 0 & 0.0 & 0.0 \\
\hline $1.2 \mathrm{E}-0 \mathrm{~L}$ & - & $1 . \theta E-01$ & 0 & 0 & 0.0 & 0.0 \\
\hline $1.8 \mathrm{E}-01$ & - & $2.6 E-01$ & 0 & 0 & 0.0 & 0.0 \\
\hline $2.6 \mathrm{E}-01$ & - & 3. $8 E-01$ & 0 & 0 & 0.0 & 0.0 \\
\hline 3. $8 \mathrm{E}-0 \mathrm{I}$ & - & $5.6 E-01$ & 0 & 0 & 0.0 & 0.0 \\
\hline $6 \mathrm{E}-01$ & - & $8.3 E-01$ & 0 & 0 & 0.0 & 0.0 \\
\hline $8.3 E-01$ & - & $1.2 \mathrm{E} \quad 00$ & 0 & 0 & 0.0 & 0.0 \\
\hline $.2 \mathrm{E} 00$ & - & $1.8 E \quad 00$ & 0 & 0 & 0.0 & 0.0 \\
\hline BE 00 & - & $2.6 \mathrm{E} \quad 00$ & 1 & 1 & 3.03 & 3.03 \\
\hline
\end{tabular}

HISTOGRAM FOR COLUMN 31 AU PPM)

$2.0 \mathrm{E} 00 \times x \times$

\begin{tabular}{|c|c|c|c|c|c|c|c|}
\hline & $\mathbb{N}$ & $L$ & $H$ & 8 & $T$ & G & $\begin{array}{l}\text { ANALYTICAL } \\
\text { VALUES }\end{array}$ \\
\hline$\infty$ & $\begin{array}{c}0 \\
0.0\end{array}$ & $\begin{array}{c}32 \\
96.97\end{array}$ & 0 & 0 & $\begin{array}{c}0 \\
0.0\end{array}$ & $\begin{array}{c}0 \\
0.0\end{array}$ & 1 \\
\hline
\end{tabular}

MAXIMUM $=2.20000 E 00$

MINIMUM $=2.20000 E 00$

GEOMETRIC MEAN $=2.20000 E 00$

GEOMETRIC DEVIATION $=9.99900 E 48$ 
ELEMENT

N

FE PCT MG PCT

CA PCT

TI PCT

MN PPM

$A G$ PPM

AS PPM

AU PPM

B PPM

A PPM

$B E$ PPM

I PPM

CD PPM

C PPM

CR PPM

CU PPM

LA PPM

$M 0$ PPM

NB PPM

I PPA

PB PPM

SB PPM

SC PPM

SN PPM

$\infty$ SR PPM

$\checkmark P P M$

W PPM

$Y$ PPM

ZN PPM

ZR PPM

AU PPM

\begin{tabular}{|c|c|c|c|}
\hline & GEOMETRIC & GEOMETRIC \\
\hline & & MEAN & DEVIATIDN \\
\hline \multicolumn{2}{|c|}{$\begin{array}{l}\text { ELEMENT } \\
\text { FE PCT }\end{array}$} & 4.893481 & 1.63 \\
\hline MG & PCT & 1.836679 & 1.33 \\
\hline$C A$ & PCT & 1.411264 & 1.37 \\
\hline$I$ & $P C T$ & 0.431725 & 1.47 \\
\hline MN & PPM & 681.222900 & 1.54 \\
\hline$A G$ & PPM & 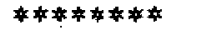 & 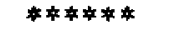 \\
\hline AS & PPM & 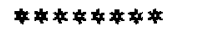 & 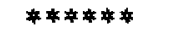 \\
\hline AU & PPM & $* * \div \div * * *$ & 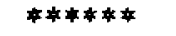 \\
\hline B & PPM & 9.322834 & 1.40 \\
\hline BA & PPH & 406.046875 & 1.83 \\
\hline$B E$ & PPM & $* * * * * * *$ & $4 * 4 * * 4$ \\
\hline BI & PPM & $* * * * * * * *$ & $\% * 4 * 4$ \\
\hline $\mathrm{CD}$ & PPM & $* * * * * * *$ & $\# \# \# \div$ \\
\hline $\mathrm{CO}$ & PPM & 19.520279 & 1.50 \\
\hline CR & PPM & 70.238785 & 2.12 \\
\hline $\mathrm{Cu}$ & PPM & 19.753891 & 2.32 \\
\hline LA & PPM & 14.243526 & 1.85 \\
\hline MO & PPM & $* * * * * * * *$ & *廿**** \\
\hline NB & PPM & 9.510991 & 1.10 \\
\hline N I & PPM & 29.576355 & 2.23 \\
\hline PB & PPM & 10.554599 & 1.70 \\
\hline
\end{tabular}

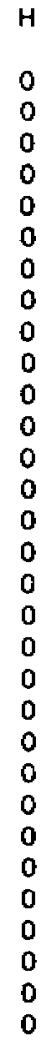

B

1
0
0
0
0
0
0
0
0
0
0
0
0
0
0
0
0
0
0
0
0
0
0
0
0
0
0
0
0
0
0
0

REMARKS

33 SAMPLES AND 33 ANALYTICAL VALUES.

33 SAMPLES AND 33 ANALYTICAL VALUES.

33 SAMPLES AND 33 ANALYTICAL VALUES.

33 SAMPLES AND 33 ANALYTICAL VALUES.

33 SAMPLES AND 33 ANALYTICAL VALUES.

33 NOT DETECTED, LESS THAN, DR TRACE VALUES.

33 NOT DETECTED, LESS THAN, OR TRACE VALUES.

33 NOT DETECTED, LESS THAN, OR TRACE VALUES.

12 NOT DETECTED, LESS THAN, OR TRACE VALUES. 33 SAMPLES AND 33 ANALYTICAL VALUES.

32 NOT DETECTED, LESS THAN, OR TRACE VALUES. 33 NOT DETECTED, LESS THAN, OR TRACE VALUES. 33 NOT DETECTED, LESS THAN, OR TRACE VALUES. 33 SAMPLES AND 33 ANALYTICAL VALUES.

33 SAMPLES AND 33 ANALYTICAL VALUES.

1 NOT DETECTED, LESS THAN, OR TRACE VALUES. 21 NOT DETECTED, LESS THAN, OR TRACE VALUES. 33 NOT DETECTED, LESS THAN, OR TRACE VALUES. 7 NOT DETECTED, LESS THAN, DR TRACE VALUES. 1 NOT DETECTED, LESS THAN, OR TRACE VALUES.
10 NOT DETECTED, LESS THAN, OR TRACE VALUES.
O REPORTED VALUES. NO COMPUTATIONS. O REPORTEO VALUES. NO COMPUTATIONS. O REPORTED VALUES. NO COMPUTATIONS. 21 REPORTED VALUES.

1 REPORTED VALUES. NO COMPUTATIONS. 0 REPORTED VALUES. NO COMPUTATIONS. O REPORTED VALUES. ND COMPUTATIONS.

32 REPORTED VALUES.

12 REPOR TED VALUES.

O REPORTED VALUES. NO COMPUTATIONS.

26 REPOR TED VALUES.

32 REPDRTED VALUES.

23 REPORTED VALUES. 


$\begin{array}{llrr}\text { SB PPM } & * * * * * * * & * * * * * \\ \text { SC PPM } & 19.056198 & 1.29 \\ \text { SN PPM } & * * * * * * * & * * * * * \\ \text { SR PPM } & 195.302475 & 1.36 \\ V \text { PPM } & 157.641693 & 1.54 \\ \text { W PPM } & * * * * * * * & * * * * * * \\ \text { Y PPM } & 17.520523 & 1.35 \\ \text { ZN PPM } & * * * * * * * & * * * * * * \\ \text { ZR PPM } & 88.247421 & 1.87 \\ \text { AU PPM } & * * * * * * * & * * * * * *\end{array}$

33 NOT DETECTED, LESS THAN, OR TRACE VALUES. 33 SAMPLES AND 33 ANALYTICAL VALUES

33 NOT DETECTED, LESS THAN, OR TRACE VALUES.

33 SAMPLES AND 33 ANALYTICAL VALUES.

33 SAMPLES AND 33 ANALYTICAL VALUES.

33 NOT DETECTED, LESS THAN, DR TRACE VALUES.

33 SAMPLES AND, 33 ANALYTICAL VALUES.

32 NOT DETECTED, LESS THAN, OR TRACE VALUES.

33 SAMPLES AND 33 ANALYTICAL VALUES.

32 NOT DETECTED, LESS THAN, OR TRACE VALUES. o reported values. no computations.

o reported values. no computations.

o RePorted VALUES. no computations.

I REPORTED VALUES. NO COMPUTATIONS.

1 REPORTED VALUES. NO CoMPUTATIONS. 